ARGONNE NATIONAL LABORATORY

9700 South Cass Avenue

Argonne, Illinois 60439

EFFECT OF ANIONIC CONSTITUENTS ON

THE SURFACE IONIZATION OF LITHIUM SALTS

by

Eric N. Sloth

Chemistry Division

Submitted in Partial Fulfillment of the

Requirements for the Degree of

Doctor of Philosophy in Chemistry

in the Graduate School of

Illinois Institute of Technology

July 1969

LEGAL NOTICE

report ana preparcd as an account of Government sponoorcd work. Netther the Untted Makes any warranty or represersintion, expreased or of the Comsmisslon:

ompleteness, or usefulness of the informatt un contnimpd in the with respect to the sccu-

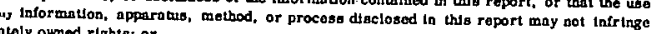
B. Assumes any

B. Assumes any llabllitues wtith reapect to the use of, or tor damages resultung from the As used in the aponaratus, method, or process disclosed in this report.

ployes or cosuractor of the "Doraon acung on behalf of the Commlaston" Includes any em-

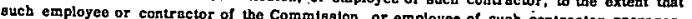

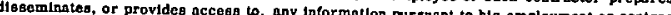
whis the Commisslon, or his employment with such contrector. 


\section{DISCLAIMER}

This report was prepared as an account of work sponsored by an agency of the United States Government. Neither the United States Government nor any agency Thereof, nor any of their employees, makes any warranty, express or implied, or assumes any legal liability or responsibility for the accuracy, completeness, or usefulness of any information, apparatus, product, or process disclosed, or represents that its use would not infringe privately owned rights. Reference herein to any specific commercial product, process, or service by trade name, trademark, manufacturer, or otherwise does not necessarily constitute or imply its endorsement, recommendation, or favoring by the United States Government or any agency thereof. The views and opinions of authors expressed herein do not necessarily state or reflect those of the United States Government or any agency thereof. 


\section{DISCLAIMER}

Portions of this document may be illegible in electronic image products. Images are produced from the best available original document. 
TABLE OF CONTENTS

$\underline{\text { Page }}$

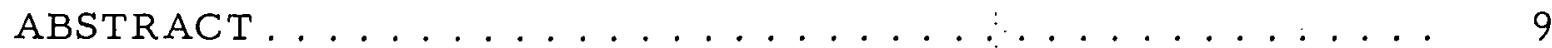

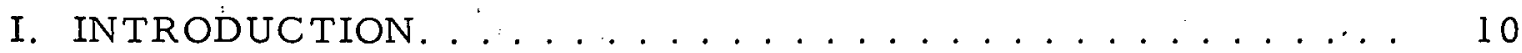

II. REVIEW OF PERTINENT EXPERIMENTAL ASPECTS OF SURFACE IONIZATION . . . . . . . . . . . . . . . 12

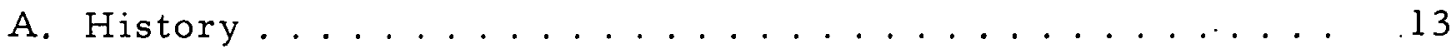

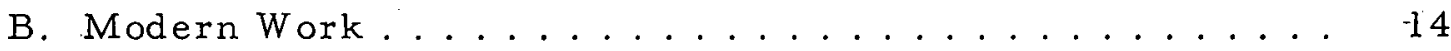

1. Work-function Variation in Metals............ 16

2. Mean Residence Times ............... 18

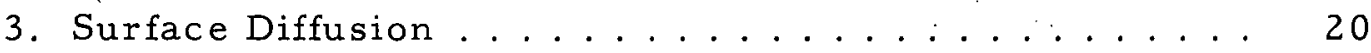

4. Adsorption States and Flash Desorption ........ 20

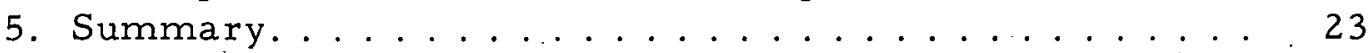

III. THEORY OF SURFACE IONIZATION . . . . . . . . . 26

A. The Saha-Langmuir Equation. . ............. 27

B. Sketch of Statistical Derivation ............. 28

C. Effect of Mean Residence Times .............. 30

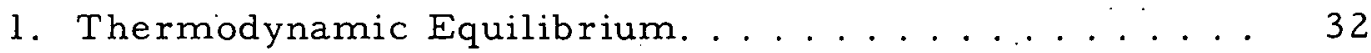

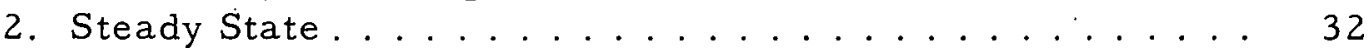

D. Influence of Anionic Constituents in the Molecular Beam . . 33

1. Complete Dissociation of $\mathrm{MX}\left(\mathrm{Z}_{\mathrm{MX}}=0\right) \ldots 38$

2. Incomplete Dissociation of $\mathrm{MX}\left(\mathrm{Z}_{\mathrm{MX}} \neq 0\right) \ldots \ldots 38$

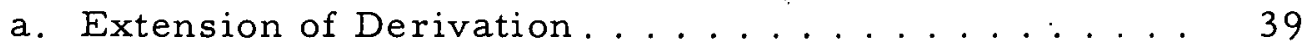

b. Compàrison with Experiment.......... 41

E. Thermodynamic Considerations . . . . . . . . . . . 44

F. Influence of Polycrystalline Surfaces . . . . . . . . . . 49

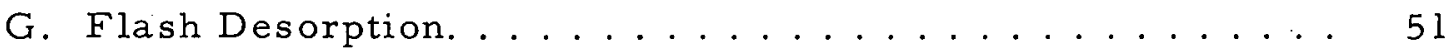

H. Recent Developments................. 56

IV. EXPERIMENTAL STUDY. ....................... 60

A. Description of Apparatus . . .............. 60

1. Time-of-Flight Mass Spectrometer ........... 60

2. Source Filaments ................ 63 
TABLE OF CONTENTS

$\underline{\text { Page }}$

3. Temperature Measurements .............. 65

a. Resistance Thermometry............. 66

(1) Calibration of Sample Filament ......... 66

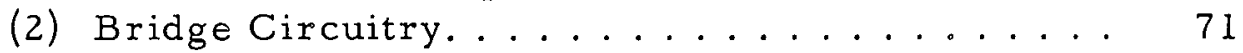

(a) Introduction ...................... 71

(b) Mathematical Analysis of the Maxwell

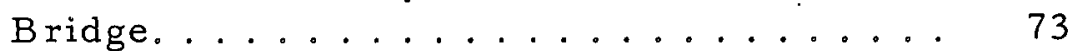

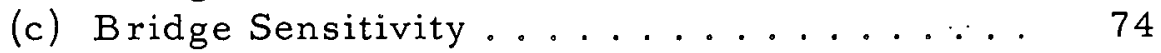

(d) Illustration of Null Detection. . . . . . 7.5

(e) Calibrätion of Resistors ......... 76

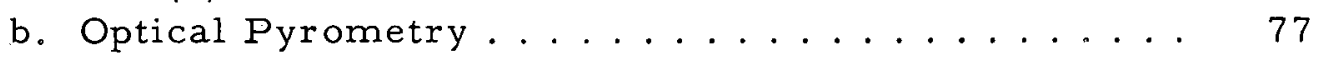

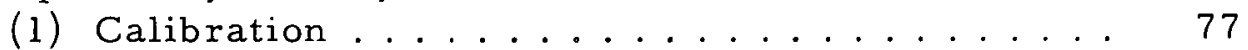

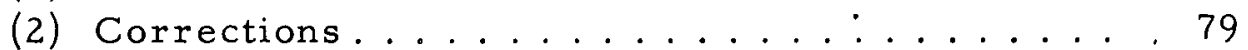

4. Flash-desorption Circuitry.............. 81

B. Experimental Prelude ................. 82

1. Materials Section ................. 82

a. Characteristics of Rhenium .......... 82

b. Preparation of Rhenium Filaments ......... 84

c. Sample Preparation ............... 84

2. Preliminary Procedures ............... 85

a. Flash Desorption of Residual Gases......... 85

b. Thermionic Work Function. . . . . . . . . . . 94

c. Surface-ionization Work Function .......... 98

d. Effect of Residual Gases on Work Function....... 101

V. RESULTS AND DISCUSSION ................. 107

A. The System Lithium Chloride on Rhenium ........ 107

B. The System Lithium Bromide on Rhenium ......... 111

C. The System Lithium Iodide on Rhenium ........... 112

D. The System Lithium Sulfate on Rhenium .......... 114

E. The System Lithium Nitrate on Rhenium ........... 117

VI. SUMMARY AND CONCLUSIONS ................ 119

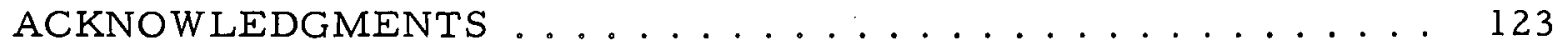

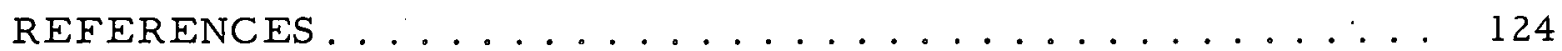




\section{LIST OF FIGURES}

No.

1. Dependence of Lithium Ion Current on Tungsten Surface

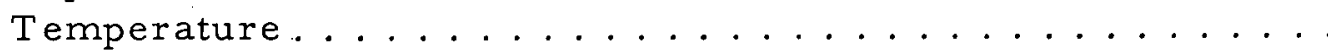

2. Reflection of Alkali Metals on a Platinum Surface ....... 15

3. Potential Energy of System Metal + Ion + Electron. . . . . . 28

4. Schematic Diagram of the Temporal Behavior of a Filament Surface Temperature and Gas Pressure............ 52

5. Bond Types for Atom-Metal System at $0^{\circ} \mathrm{K} \ldots \ldots . \ldots 58$

6. Relation between Broadened Atom Level and Fermi-Dirac Distributed Electrons for $\mathrm{T}>0^{\circ} \mathrm{K} \ldots \ldots \ldots 58$

7. Operation of Blanking Generator...............663

8. Mercury Spectrum ..................... 63

9. Recorder Scan of Mercury with Ion Source Tuned for

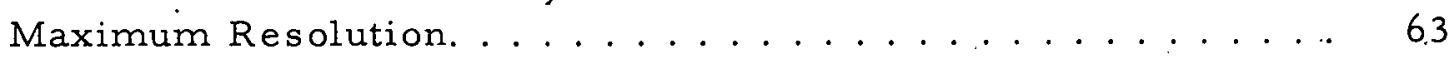

10. Spectra of Alkali Metals Li $(6,7), \mathrm{Na}(23)$, and $\mathrm{K}(39,41)$ from Surface Ionization on Rhenium ................ 65

11. Temperature vs Bridge Resistance . . . . . . . . . . . 69

12. Maxwell Bridge and Associated Null-detection Circuitry . . . 73

13. Illustration of Bridge Balancing ............. 75

14. Bridge Null Condition with a 180-Hz Harmonic Present. . . . 76

15. Circuitry for Flashing Filament .............. 81

16. Fraction Ionized $\dot{s}$ 'Temperature for the Alkali Metals as a Function of the Parameter. $\Delta=I-\phi \ldots \ldots 84$

17. Variation in Bridge Unbalance during Flashing ......... 90

18. Mass 28 Voltage Pulse during Flashing ............ 90

19. Drop in $\mathrm{N}_{2}^{+}$: Ion Current vs. Time after.Flow Stoppage..... . 91

20. Mass 28 Ion-current Pulse Height vs Cooling Timè . . . . . . 91

21. Schematic Diagram of Arrangement for Measurement of Thermionic Work Function. ............... 96

22. Thermionic Work Function of Rhenium. . . . . . . . . 97

23. Vapor Pressure of Lithium Metal vs Reciprocal

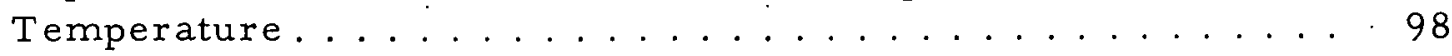




\section{LIST OF FIGURES}

No.

Title

$\underline{\text { Page }}$

24. Background Lithium Ion Current from Rhenium Filament . . . 99

25. Temperature Dependence of ${ }^{7}$ Li Ion Current .......... 100

26. Temperature Dependence of Lithium Ion Current for Clean and Contaminated Surfaces................. 102

27. Approach of Lithium Ion Current to Equilibrium for

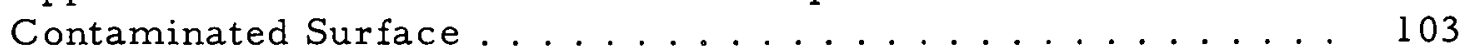

28. Temperature Dependence of Composite Rate Constants . . . . 106

29. Vapor Pressure of Lithium Chloride vs Reciprocal

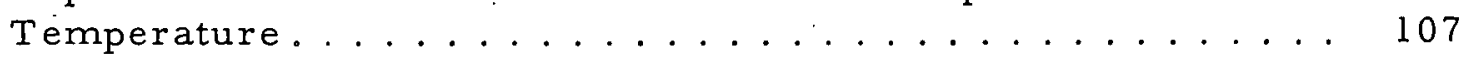

30. Temperature Dependence of the Surface Ionization of Lithium Chloride ................... 108

3.1. Temperature Dependence of Surface Ionization on Rhenium . . 109

32. Dissociation-energy Data for Lithium Chloride . . . . . . . 110

33. Temperature Dependence of the Surface Ionization of Lithium Bromide.................... 111

34. Dissociation-energy Data for Lithium Bromide ........ 112

35. Temperature Dependence of the Surface Ionization of Lithium Iodide...................... 113

36. Temperature Dependence of the Lithium Surface-ionization Current for Lithium and Dilithium Oxide on Rhenium . . . . . . 117

37. Temperature Dependence of the Lithium Surface-ionization Current for the System Lithium Nitrate on Rhenium. . . . . . . 
LIST OF TABLES

No.

Title

Page

I. Experimental Data for the Work Functions of Different Crystal Planes for Tungsten. ..............

II. Effect of Surface Coverage on Work Function of Tungsten...

III. Monolayer Adsorption Times for Gases and Vapors at Different Pressures . . . . . . . . . . . . . . .

IV. Degree of Ionization, $\gamma$, vs Temperature: for Chlorine $\therefore \quad \ldots .$.

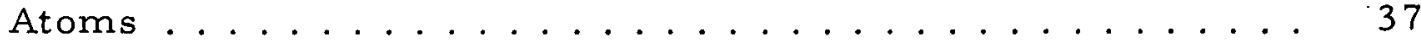

V. Variation in Fraction $f$ of LiF Dissociated as a Function of Temperature for Different Work Functions, $\phi$, and Incident-beam Intensities, $\mathrm{Z} \ldots \ldots \ldots$

VI. Values and Errors of the Temperature Coefficients in the Power Series Relating Temperature to Resistance.......

VII. Temperature vs.: Bridge Resistance........... 68

VIII. Resistivity of Rhenium, from Caldwell. . . . . . . . . . 69

IX. Out-of-balance Voltage Expected for an Imbalance of \pm 0.005 ohm in $R_{v}$ at Various Values of $R_{V} \ldots \ldots \ldots$

X. Sticking Probability and Saturation Surface Coverage of Gases on Polycrystalline Rhenium Filaments ......... 93

XI. Emission Constants of Rhenium ............... 97 


\title{
EFFECT OF ANIONIC CONSTITUENTS ON THE SURFACE IONIZATION OF LITHIUM SALTS
}

\author{
by
}

\author{
Eric N. Sloth
}

\begin{abstract}
The validity of the Saha-Langmuir theory of surface ionization was demonstrated for the lithium-rhenium system over a temperature range $1200-2600^{\circ} \mathrm{K}$. The effective surface-ionization work function obtained for rhenium was $5.21 \pm 0.01 \mathrm{eV}$, compared to the measured thermionicemission work function of $4.98 \pm 0.03 \mathrm{eV}$. At relatively high temperatures, the temperature dependence of the lithium surface-ionization current from all molecules studied was identical to that obtained from lithium atoms. Incomplete dissociation of lithium chloride and lithium bromide can account for the lithiumionization threshold temperatures being well above that for surface ionization of lithium atoms. Dis sociation energies of $\mathrm{D}_{\mathrm{LiCl}}(\mathrm{g})=4.9 \pm 0.1 \mathrm{eV}\left(1300-1700^{\circ} \mathrm{K}\right)$ and $\mathrm{D}_{\mathrm{LiBr}}(\mathrm{g})=4.4 \pm 0.1 \mathrm{eV}\left(1150-1400^{\circ} \mathrm{K}\right)$ were obtained. Enhanced emission of lithium ions was. observed in the temperature range where incomplete dissociation of lithium iodide was expected due to a rhenium-iodine interaction shifting the dissociation equilibrium to lower temperatures. For lithium nitrate incident on rhenium, several lithium rhenates were formed corresponding to $\mathrm{Li}_{2} \mathrm{ReO}_{4}, \mathrm{LiReO}_{4}$, and $\mathrm{LiReO}_{3}$. Lithium sulfate decomposed to give beams of dilithium oxide and lithium atoms. The temperature dependence of the lithium surface-ionization current was the sum of the individual ion currents from lithium atoms and dilithium oxide. A desorption energy of $4.13 \mathrm{eV}$ was obtained for residual-gas oxygen on rhenium using surface-ionization techniques.
\end{abstract}




\section{CHAPTER I}

\section{INTRODUCTION}

When atoms or molecules from a molecular beam or. from a vapor impinge on an incandescent metal surface, positive and/or negative ions may be formed at the surface and, after a mean residence time, may evaporate partly as atoms and molecules and partly as positive or negative ions. In recent years, the developments in surface ionization have followed two paths: (1) an extension of classes of atoms and metal surfaces for which surface ionization occurs and the Saha-Langmuir equation is valid, and (2) the study of the mechanism of surface ionization by direct determination of desorption and charge-transfer probabilities.

A review of atomic, molecular, and ionic impact phenomena on surfaces is provided by Massey and Burhop ${ }^{1}$ together with the summaries by Kaminsky, ${ }^{2}$ Hasted, ${ }^{3}$, and Ehrlich. ${ }^{4}$

Surface-ionization sources are used extensively in mass spectrometry for isotope abundance determinations in solid samples. ${ }^{5}$. Variations among individual mass analyses, which depend on sample treatment and technique of analysis, have emphasized the need for a better understanding of pertinent chemical effects. For example, we have demonstrated that by control of the oxidizing and reducing agents on an electrically heated filament and in the surrounding atmosphere, the species of emitted uranium ions can be regulated. 6 In his study of the surface ionization of strontium, barium, neodymium, and uranium on hot surfaces of rhenium, tantalum, and tungsten, Werning ${ }^{7}$ found that below $2000^{\circ} \mathrm{K}$ each system has its own chemical properties. Zandberg and Tontegode ${ }^{8}$ investigated the temperature dependence of the surface-ionization currents of alkali-metal atoms and molecules of their chloride salts on polycrystalline rhenium wire. They found that, above a certain threshold temperature, the temperature dependence of the surface-ionization currents of atoms and the corresponding molecules of the salt are identical; this temperature increases in the series of alkali metals from cesium to lithium. The threshold ionization temperatures for atomic beams are lower than for the corresponding molecular beams, the difference of these temperatures likewise increasing from cesium to lithium. These results are readily explained on the basis of complete dissociation of the molecules of the salts on rhenium in the hightemperature region and reduced dissociation of the molecules in the region of threshold temperatures. The conclusion to be drawn from these studies is that each surface-ionization system has within it chemical characteristics that must be resolved on an individual basis rather than by recourse to general surface properties such as bulk work functions, surface inhomogeneities, or reflection coefficients.

The effect of anionic constituents on the surface ionization of selected lithium-salts on polycrystalline rhenium filaments is investigated in the 
present work. It is shown experimentally that the threshold temperature . for lithium ion emission in the surface ionization of lithium halides can be correlated with the dissociation energy of the halide. When certain experimental conditions are fulfilled, the dissociation energy of the lithium halide can be uniquely determined. Enhanced emission of lithium ions is observed when iodine is the anionic constituent of the lithium salt. Lithium nitrate interacts with the rhenium substrate to produce complex gaseous molecules containing substrate atoms.

The pertinent experimental and theoretical aspects of surface ionization are reviewed briefly in Chapters II and III. The questions of adsorption, surface diffusion, mean residence times, surface states, and desorption are considered.

A combination of ultrahigh vacuum, flash desorption, and mass spectrometry was applied to this study. The equipment used is described in detail.

- The experimental data are presented and analyzed, both with respect to the observed general features of surface ionization of lithium under the conditions of these experiments and with respect to the previous concepts and observations by others. 


\section{CHAPTER II \\ REVIEW OF PERTINENT EXPERIMENTAL ASPECTS OF SURFACE IONIZATION}

Experiments describing the measurement of ion emission from heated metal surfaces date back to $1903 .^{9}$ The general nature of thermal surfaceionization phenomena was known before 1950. However, a characteristic of . the early experimental work is the large fluctuations in the experimental data and the lack of reproducible results.

The principal contributing factor was the inability to maintain a clean system. Residual gases present in the vacuum system played an important, but largely-unrecognized, role in the ion-emission properties of a heatedfilament surface. More recent studies, ${ }^{10-13}$ using ultrahigh-vacuum techniques and modern instrumentation, indicate a variety of possible gas surface interactions affecting ion emission. Some of these reactions are as yet not adequately understood.

The data obtained are generally analyzed in terms of the SahaLangmuir equation,

$$
\alpha=I^{+} / I_{0}=\frac{g_{+}}{g_{0}} \exp \frac{\phi-I}{k T},
$$

where $\alpha$ is the degree of ionization, $\mathrm{I}^{+}$is the ion particle current, $\mathrm{I}_{0}$ is the neutral atom current, $g_{+} / g_{0}$ is the ratio of the statistical weights of the ionic and atomic states, $\phi$ is the electronic work function of the surface, I is the ionization potential of the emitted particles, $k$ is Boltzmann's constant, and $T$ is the surface temperature. The data are usually plotted as $\log \mathrm{I}^{+}$ versus $1 / T$. The value obtained from the slope of the curve, usually a straight line, is then subject to various interpretations. The few cases in which quantitative agreement has been obtained have been limited to those alkali metals of low ionization potential evaporating from tungsten surfaces. Here, however, the efficiency of the process is so great that the Saha Langmuir type of ionization swamps out any other mechanism that might compete.

Deviations from the Saha-Langmuir theory have been attributed to the nonhomogeneity of the crystalline surface, to the effect of adsorbed fractional layers of residual gases on the work function of the surface, to reflection coefficients, to chemical interaction between the incident particles and impurities present in the refractory metal surfaces, and to the possibility that the Saha-Langmuir theory does not account for the extra $\cdot$ valence electrons encountered in cases other than the alkali metals. Corrections have been made in an attempt to take account of such deviations... The "corrected" equation for the degree of ionization, $\alpha$, in zero external electric field is 


$$
\alpha=\frac{G_{+}}{G_{0}} \cdot \frac{1-r_{+}}{l-r_{0}} \exp \frac{a}{k} \exp \frac{\Psi-I}{k T},
$$

also derived by Dobrezow ${ }^{14}$ from statistical arguments. Here $G_{+}$and $G_{0}$ are the statistical weights for ions and atoms in which the latter can also occur in an excited state whose excitation energy is comparable to $\mathrm{kT}$. The quantities $r_{+}$and $r_{0}$ are the reflection coefficients ${ }^{15}$ of the surface for ions and atoms, respectively. The quantities $\mathrm{a} / \mathrm{k}$ and $\Psi$ account for the temperature dependence of the work function, $\phi$, defined by $\phi(\mathrm{T})=\phi\left(\mathrm{T}_{0}\right)+$ $\mathrm{a}\left(\mathrm{T}-\mathrm{T}_{0}\right)$ and $\Psi=\phi\left(\mathrm{T}_{0}\right)-\mathrm{a} \mathrm{T}_{0}$. For homogeneous surfaces, this temperature dependence of the work function can generally be neglected. The effect of an external electric field of sufficient magnitude to cause a change in the work function is not applicable to this report.

\section{A. History ${ }^{16}$}

For an atomic gas in contact with a solid surface, three processes ma.y be considered: adsorption, surface diffusion, and evaporation. These processes were first analyzed, for the interaction of cesium with tungsten wires by Taylor and Langmuir, ${ }^{17}$ continuing the earlier studies of surface ionization by Langmuir and Kingdon. ${ }^{18}$ When a cesium atom impinges on a tungsten surface, the collision results in adsorption with a sticking probability of unity, independent of surface concentration or gas temperature, up to concentrations at which the entire surface appears to be filled with a layer of adatoms. This invalidated Langmuir's earlier picture of the adsorption process ${ }^{19}$ in which the rate of adsorption decreased with increasing surface concentration as the fraction of unoccupied sites $(1-\theta)$ diminished.

The capture rate is equal to the rate of impingement from the gas phase, even at high coverages, implying that cesium atoms must be able to exist in a weakly bound state when not interacting with the surface directly. Evidently an encounter with a filled site does not result in reflection. The incoming atom, however, succeeds in finding an empty site where it can be permanently bound before evaporating from this transition state.

The desorption of cesium is complicated by the fact that it can evaporate either as a neutral atom or as an ion, leaving an electron on the surface. Since there is no barrier to adsorption, the desorption energy is equivalent to the heat of adsorption. The energy of a cesium atom is lowered by $64.3 \mathrm{kcal} / \mathrm{mole}$, relative to the isolated gas -phase atom at $\mathrm{T}=0 \mathrm{~K}$, when it is trapped by the tungsten surface. This is a considerably larger change than taking an atom from the gas phase to the surface of solid cesium $(18.7 \mathrm{kcal} / \mathrm{mole})$.

In contrast, the barrier to diffusion of adsorbed cesium is only onefifth of the barrier to evaporation of a cesium atom at the same surface concentration. The concentration must be specified since the desorption 
energy for adatoms decreases sharply as the surface becomes more densely populated with cesium. At low temperatures, where $\mathrm{kT}$ is small in comparison with the barrier to migration, the adatoms are localized at positions of minimum energy. At high temperatures, the energy differences between different positions become washed out, and the adsorbed layer approaches the behavior of a two-dimensional gas.

\section{B. Modern Work}

The general features outlined for the adsorption-desorption processes of cesium on tungsten are found also in many other systems. Zandberg and Ionov ${ }^{20}$ and Kaminsky ${ }^{2}$ have presented thorough and critical reviews of the experimental work in surface ionization.

The most popular ionization surface was polycrystalline tungsten, though many authors of earlier works did not consider the "patch" character of the surface in discussing their results. The influence of foreign-gas coverage of the surface was likewise disregarded. The small value for the degree of ionization, $\alpha$, for lithium on tungsten makes it clear why the corresponding experimental values could be found only recently with improved systems for ion detection.

For this case, the ionization potential is much greater than the work function, so that the ion current, $\mathrm{I}^{+}$, follows the Saha-Langmuir equation, with the simplification that $I_{0}$, the atom "current," is essentially constant; i.e.,

$$
\mathrm{I}^{+}=\mathrm{I}_{0} \mathrm{~A} \exp \frac{\phi-\mathrm{I}}{\mathrm{kT}}
$$

From this, it follows that the logarithm of the ion current falls off linearly with $1 / k T$, and that the difference $\phi-I$ is the negative slope of the line. Romanov ${ }^{21}$ and $Z$ andberg and Ionov ${ }^{22}$ have verified this relation experimentally for the high-temperature region of $2100-2500^{\circ} \mathrm{K}$ (as shown in Fig. 1 ).

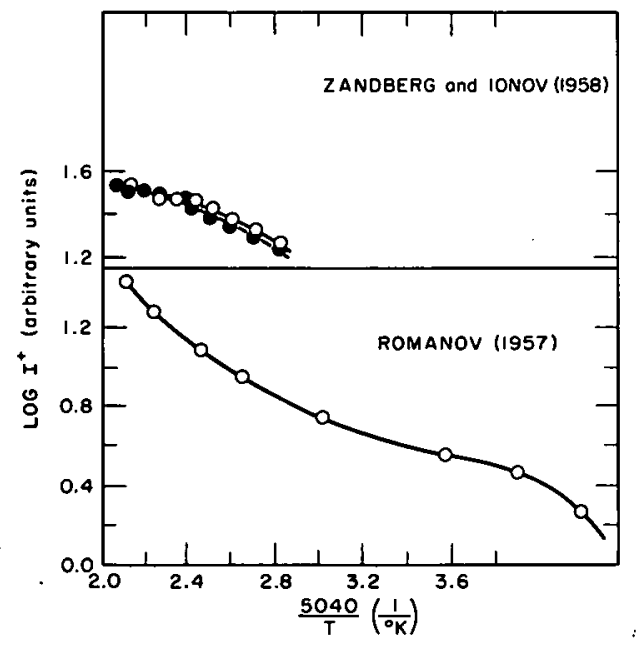

Fig. 1

Dependence of Lithium Ion Current on Tungsten Surface Temperature: 
At lower surface temperatures, they found deviation from linearity. Romanov ${ }^{21}$ observed that the curve of $\log \mathrm{I}^{+}$versus $1 / \mathrm{kT}$ at low temperatures passes through a slight maximum. The occurrence of such a maximum was explained qualitatively as a result of oxygen (which raises the work function) and lithium (which lowers the work function) competing for the adsorption sites.

Zandberg and Ionov ${ }^{20}$ presented theoretical arguments concerning the influence of the patch character of the surface on the ion current. Unfortunately, in applying such arguments to the interpretation of their measurements, they failed to consider that the average value of the work function of a polycrystalline surface as obtained from measurements of thermionic emission is not identical with the value from positive surfaceionization measurements. In thermionic emission, electrons are emitted from patches where $\phi_{\text {min }}$ dominates; in surface ionization, the patches with $\phi_{\max }$ dominate.

In the surface ionization of lithium on pure platinum and on $92 \%$ platinum-8\% tungsten, Datz and Taylor ${ }^{11,23}$ found that the degree of ionization was much smaller than expected and the slope of the Saha-Langmuir line did not agree with the theoretically expected value (as plotted in Fig. 2). The authors suggested that a certain fraction of the particles striking the surface are reflected without coming into thermal equilibrium. However, the temperature dependence of the reflection coefficient (a 100\% increase

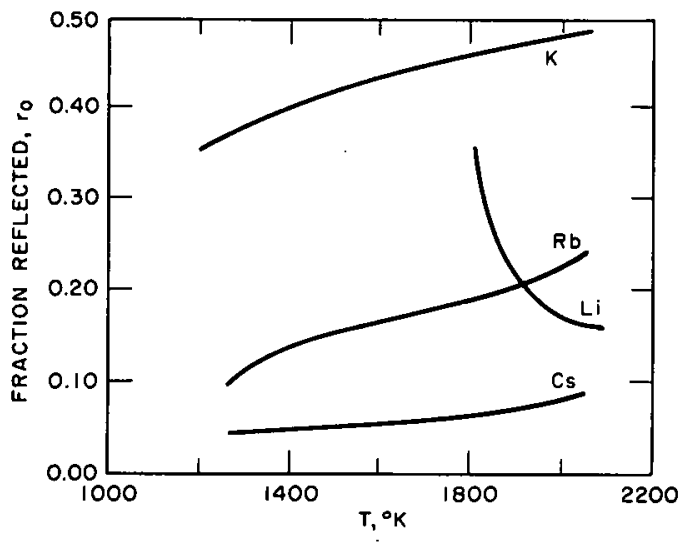

$121-5487$

Fig. 2. Reflection of Alkali Metals on a Platinum Surface between 1800 and $2000^{\circ} \mathrm{K}$ ) given by these authors is improbably large for a reflection process. Pong and Sokolskaya ${ }^{24}$ attributed their low value for the degree of ionization of sodium on platinum to the development of highly chemisorbed sodium layers on the platinum surface, which hinder the ionization process because of their semiconductor character. A more likely explanation is that the absorbed alkali layers lower the work function of the metal and thus decrease the degree of ionization. ${ }^{2}$

Zandberg and Tontegode ${ }^{8}$ inves tigated the surface ionization of lithium on rhenium and found it to obey the SahaLangmuir theory. Kaminsky ${ }^{25}$ studied the mean residence time of the alkali ions on polycrystalline tungsten surfaces using modulated atomic beams with phase-sensitive mass-spectrometric detection. His results are discussed in Section B.2 below.

Considerable experimental information is available about the ionization of alkali-salt molecules on metal surfaces. In general, the 
high-temperature dependence of the current of alkali ions is largely independent of whether the alkali is in the form of metal atoms or alkali-salt molecules impinging on tungsten surfaces. Experimental studies at high temperatures have shown that the alkali-salt molecules incident on hottungsten surfaces are dissociated and come to thermal equilibrium, and only then is the alkali ion desorbed.

Zandberg and Tontegode ${ }^{8}$ observed the temperature dependence of the surface-ionization currents of alkali atoms and molecules of their chloride salts on polycrystalline rhenium wire. Above a certain temperature, the temperature dependence of the surface-ionization currents of atoms and the corresponding chloride salts was the same; this temperature increases in the series of alkali metals from cesium to lithium. The threshold ionization temperatures for the atomic beams were lower than for the molecular beams. The difference of the threshold temperatures likewise increases from cesium to lithium. The results are explained by complete dissociation of the molecules of the salts on rhenium in the high-temperature region and incomplete dissociation of the molecules in the threshold-temperature region. Combining ionization of lithium atoms and lithium chloride molecules showed the total current to be the sum of the currents corresponding to the atomic and molecular beams. The temperature relationships obtained for the surface-ionization currents of alkali-metal atoms on rhenium corresponded qualitatively to those predicted by the theory of surface ionization on inhomogeneous surfaces.

For platinum surfaces, the temperature dependence of the degree of ionization varies strongly with the kind of potassium halide salt incident on the surface. Again, the values of $\alpha$ are far below those expected from the Saha-Langmuir equation. Datz and Taylor ${ }^{23}$ interpreted these results as being due to reflection, as for potassium atoms on platinum surfaces.

\section{Work-function Variation in Metals}

The Saha-Langmuir equation is strictly valid only for a clean homogeneous surface. However, most investigations of surface ionization have been carried out on polycrystalline metal surfaces and frequently with an unknown covering of foreign gas. The influence of physical, chemical, and induced heterogeneity on the work function, and consequently on the degree of ionization, is briefly considered.

Physical heterogeneity arises from deviations of an imperfect crystal from the strict periodic arrangement of an ideal crystal. When ions move to interstitial positions (Frenkel defects) or to the surface of a crystal (Schottky defects), their previous positions are left vacant. Dislocations, consisting of slight tilts or displacements of one region of the crystal relative to another one, are also common. These defects and dislocations can be expected to occur on a crystal surface as well, giving rise to different binding forces, which are exerted on a given particle by its neighbors. ${ }^{26}$ 
Different crystal planes on a metal surface have a marked effect on the work function. The greater the number of nearest neighbors and the shorter the distance between them, the greater the work function. Table I lists the work functions of some individual crystal planes of tungsten, as determined by thermionic emission.

TABLE I. Experimental Data for the Work Functions of Different Crystal Planes for Tungsten

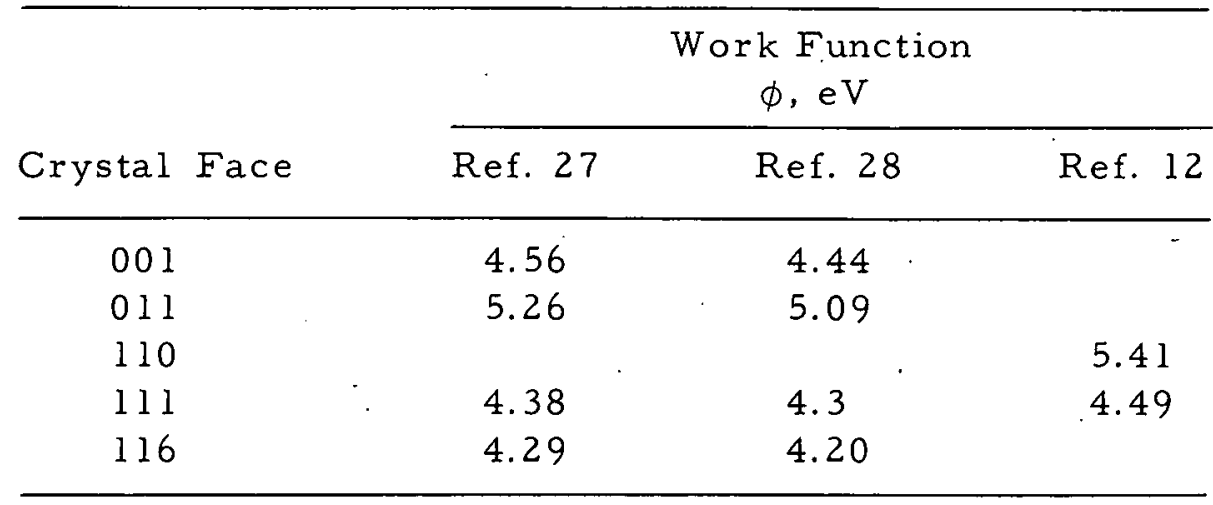

Imperfection in a crystal as a result of foreign atoms in the host lattice is termed chemical heterogeneity. These impurities can wander through the crystal lattice and arrive at the surface. This effect is readilynoted in the emission of potassium ions from hot-tungsten filaments. These impurities may serve as active centers in adsorption and catalytic processes affecting the value of the work function.

Induced heterogeneity arises as a result of adsorbing a layer of . foreign atoms on the surface from the surrounding gas. Assuming a background pressure of $10^{-6}$ Torr, a sticking probability of unity, and the appropriate number of adsorption sites, a monatomic layer of foreign atoms would be adsorbed onto the surface in about 1 sec. This agrees with the experimental results of Becker and Hartman ${ }^{29}$ and Hagstrum. ${ }^{30,31}$

Physical adsorption is characterized by Van der Waals forces giving rise to heats of adsorption in the range of $0-5 \mathrm{kcal} / \mathrm{mole}$. Covalent forces cause a weak chemisorption bond with heats of adsorption less than about $50 \mathrm{kcal} / \mathrm{mole}$. Strong chemisorption is associated with the transfer of an electron from the adatom to the metal, or vice versa, with heats of adsorption greater than $50 \mathrm{kcal} / \mathrm{mole}$. Table II illustrates some of these effects. A comprehensive survey of these effects was published by Eberhagen. ${ }^{32}$ By convention, the negative sign indicates that a dipole layer is formed with the negative charge away from the metal although the work function is increased. 
TABLE II. Effect of Surface Coverage on Work Function of Tungsten ${ }^{2}$

\begin{tabular}{lcc}
\hline & $\begin{array}{c}\text { Fraction of } \\
\text { Surface Covered, } \theta\end{array}$ & $\begin{array}{c}\text { Change in } \\
\text { Work Function, } \\
\Delta \phi, \mathrm{eV}\end{array}$ \\
\hline $\begin{array}{l}113 \text {-plane Tungsten } \\
\text { Hydrogen covered }\end{array}$ & 1 & -0.40 \\
Nitrogen covered & 1 & -0.35 \\
CO covered & 1 & -0.86 \\
Polycrystalline Tungsten & & \\
\hline Oxygen covered & & -1.9 \\
Cesium & 1 & 1.31 \\
Cesium & 0.167 & 1.82 \\
Cesium & 0.263 & 2.22 \\
Cesium & 0.345 & 2.41 \\
\hline
\end{tabular}

In other studies, under unequivocally clean conditions and low temperatures, small amounts of nitrogen adsorbed on tungsten lowered the work function. ${ }^{43}$ Independent observations on different crystal planes by field-emission microscopy $y^{34-36}$ and by thermionic emission ${ }^{37}$ have confirmed the original results. Field-emission microscopy also has revealed variations in the sign of the dipole of nitrogen adsorbed on different planes. These results have not yet been adequately explained. The empirical rule that adsorption on smooth surfaces lowers the work function, whereas on rougher surfaces, in which adatoms fit more closely into the lattice, the work function increases, is open to question. ${ }^{38}$ These are interesting effects that require further study.

\section{Mean Residence Times}

When atoms or molecules from a molecular beam or vapor impinge on an incandescent surface, they evaporate, partly as neutral atoms or molecules and partly as positive or negative ions, after a mean residence time $\tau_{0}$ or $\tau_{i}$, respectively. Direct measurements of these residence times as a function of the surface temperature allow a determination of the desorption energies, $E_{0}$ and/or $E_{i}$, given by Frenkel's equation ${ }^{39}$

$$
P_{i, 0}=\frac{1}{\tau_{i, 0}}=\frac{1}{\tau_{i, 0}^{0}} \exp \left(-\frac{E_{i, 0}}{k T}\right) .
$$

For nonactivated adsorption, the mean residence time $\tau_{i, 0}$ of the adsorbed particles on the surface are inversely proportional to the 
desorption probabilities, and the quantities $\tau_{i}^{0}$ and $\tau_{0}^{0}$ can be considered to be inversely proportional to the transition probability of one of the pos sible quantum transitions in the energy exchange between the adsorbate and the surface atoms of the target lattice. The energy $E_{i}$ for ion desorption may be related to the energy $E_{0}$ for desorption of an atom through the equation $40-42$

$$
E_{0}=E_{i}+\phi-I-\Delta Q,
$$

where $I$ is the ionization potential of the adsorbed particle, $\phi$ is the electronic work function of the metal surface, and $\Delta Q$ is the heat of activation (surface-ionization potential) for the charge-transfer process that occurs between the adsorbed particle and the metal surface.

The existence of different surface states for the adsorbed particles, implied by Eq. 2.5, has been criticized by others. ${ }^{14,43-45}$ In particular, the time for an electronic transition between an adsorbed particle and a metal surface at interatomic distances is orders of magnitude less than the range of values for the mean residence time of adsorbed alkalimetal atoms for surface temperatures of $1000-1800^{\circ} \mathrm{K}$. This suggests that $\triangle Q$ approaches zero. Hence, studies of desorption processes should offer no possibility of distinguishing between different surface states for the adsorbed species.

Earlier work by various authors on the same system has given $\tau_{i}$ differing by several orders of magnitude, as well as significant variations in the desorption energies. These results have been reviewed by Kaminsky, ${ }^{46}$ and the deviations have been attributed to lack of control of the relevant parameters that influence the determination of the residence times.

Recent results on the alkali metals are particularly interesting, as they define the effect of contamination on the desorption process. Scheer and Fine ${ }^{4,48}$ measured the evaporation of cesium ions from tungsten and rhenium by a pulsed-beam technique. They noted that $\tau_{i}$ had different values, depending on whether the surface was clean or contaminated by residual gases. The mean residence time was also different when cesium iodide or cesium chloride was used to impinge on clean targets. Hughes and Levinstein ${ }^{49,50}$ observed no significant differences among the values of $\tau_{i}$ for beam particles of rubidium, rubidium chloride, or rubidium iodide impinging on clean targets, but did observe a variation when the target was contaminated by residual gases.

Kaminsky ${ }^{25}$ likewise observed variations in mean residence times and desorption energies for ions of sodium, potassium, and rubidium, on polycrystalline tungsten wires. The variations were affected by the atomic and molecular composition of the beam (metal or metal chloride) as well as by whether the tungsten was atomically clean or covered by residual gases. 
The presence of extraneous adsorbed material has two effects:

It lowers the desorption energy, and it lowers the frequency factor, $1 / \tau_{i}^{0}$. The lower frequency can arise through a reduction in the sticking probability or through an increase in the vibrational partition function of the adatoms. On clean surfaces, these complications are absent. The details of atomic processes on solids are still not available, but the increasing use of electron diffraction, molecular beams, and ion-microscopy techniques provide an eventual hoped for solution.

\section{Surface Diffusion}

Many groups are using the field-emission microscope invented by Müller ${ }^{51}$ to study the migration of adatoms on clean surfaces of metals. Since adsorption changes the rate of tunneling of electrons out of a metal in a high electric field, the adsorbed layer and its redistribution from an initially localized deposit can readily be followed. The high resolution of the microscope makes it possible to distinguish the nature of the boundary and the dependence of the diffusion on the surface of the crystal.

For rare gases, metal atoms, carbon monoxide, and electronegative atoms such as hydrogen, oxygen, and nitrogen on tungsten surfaces, diffusion over the surface becomes important at temperatures much below those required for rapid desorption. ${ }^{16}$ Evaporation, under most circumstances, is therefore likely to occur from an equilibrium distribution of adatoms.

A map of the potential field acting on the adsorbed atoms can also be provided from migration studies. Since the barrier to diffusion is small, the binding of atoms on a metal is essentially continuous over the surface. Although the binding energies of atoms at different spots on the surface differ, these differences are relatively small, and the binding is not localized at discrete sites widely separated from one another. ${ }^{52}$

\section{Adsorption States and Flash Desorption}

One goal in the experimental study of surface ionization has been to conduct the investigation with atomically clean surfaces. This has been difficult in the past since a monolayer of foreign atoms or molecules will form on an initially clean surface in approximately $l \mathrm{sec}$ at an equilibrium pressure of $10^{-6}$. Torr. Table III gives the estimated monolayer adsorption times for different background pressures and various foreign gases or vapors at room temperature. A sticking probability of unity and a clean surface with $10^{14}$ adsorption sites per square centimeter available has been assumed. For purposes of illustration, the fact that larger molecules generally occupy larger sites has been ignored. The vacuum problem then becomes one of obtaining a monolayer adsorption time sufficiently long that measurements with a clean surface are practicable. 
TABLE III. Monolayer Adsorption Times for Gases and Vapors at Different Pressures

\begin{tabular}{llc}
\hline & \multicolumn{2}{c}{$\begin{array}{c}\text { Monolayer Adsorption } \\
\text { Time, sec }\end{array}$} \\
\cline { 2 - 3 } $\mathrm{Gas}$ & $10^{-6}$ Torr & $10^{-9}$ Torr \\
$\mathrm{H}_{2} \mathrm{O}$ & 0.21 & 210 \\
$\mathrm{~N}_{2}$ & 0.26 & 260 \\
$\mathrm{O}_{2}$ & 0.28 & 280 \\
$\mathrm{CO}_{2}$ & 0.33 & 330 \\
$\mathrm{Hg}$ & 0.70 & 700 \\
\hline
\end{tabular}

Thermal desorption is frequently used to ensure clean surfaces. Here a metal surface is heated under vacuum to such a high temperature that physically and chemically adsorbed species are desorbed. The method is confined to refractory materials since at the high temperatures required most materials evaporate rapidly or even melt. A disadvantage of the heating process is that any foreign atoms present in the lattice may diffuse to the surface.

The simplicity present in the properties of a dilute surface layer at high temperatures is largely lost at lower temperatures. Here surface diffusion and the subsequent approach to an equilibrium distribution are slow. As a result of flash-desorption studies, it is apparent that molecular gases are chemisorbed on a surface in a multiplicity of states, each with discrete binding energies. The idea of multiple binding states is not new. It has been invoked to rationalize apparently anomalous work-function changes at high surface concentrations. ${ }^{53}$

A quantitative measure of contamination of a filament surface can be obtained using the technique of flash desorption, first suggested by Apker. ${ }^{54}$ Hagstrum ${ }^{30}$ used the method to ensure a clean surface in his study of electron ejection from ion-bombarded solid surfaces. The technique was first applied to chemisorption studies by Becker and Hartman ${ }^{29}$ for the nitrogen-tungsten system. Gomer ${ }^{55}$ briefly reviewed the method as applied to the study of chemisorption.

The method consists of: (1) following the pressure changes when a ribbon or wire exposed to a low pressure of gas is heated, causing desorption, or (2) allowing a thermally cleaned surface to adsorb gas at a fixed temperature. The instantaneous desorption rate per unit area, $\mathrm{d} \sigma / \mathrm{dt}$, is related to the pressure rise from equilibrium, $\Delta \mathrm{p}=\mathrm{p}-\mathrm{p}_{\mathrm{eq}}$, and its time derivative by the expression 


$$
\frac{\mathrm{AkT}}{\mathrm{V}} \frac{\mathrm{d} \sigma}{\mathrm{dt}}=\frac{\mathrm{dp}}{\mathrm{dt}}+\frac{\Delta \mathrm{p}}{\tau}+\frac{\mathrm{AkTsp}}{\mathrm{V}(2 \tau \mathrm{mkT})^{1 / 2}}
$$

provided the gas flow into the reaction system is constant. The quantity $A$ is the surface area of the ribbon, $V$ the volume of the system, s the sticking coefficient of the gas on the ribbon, and $\tau=V / S$ the mean residence time of the gaseous species in the system, $S$ being the pumping speed of the system in volume units per second. Equation 2.6 is derived in Section III.G.

If readsorption on the filament as represented by the last term in Eq. 2.6 can be neglected during desorption, and if the characteristic pumping time, T of any pumps and/or walis remains constant, Eq. 2.6 can be used directly to obtain desorption rates from the change in pressure. The expression can be integrated with respect to time to obtain the surface coverage. The rate constants and orders of the desorption reaction can be obtained directly from the instantaneous rates and coverages ${ }^{56}$ and less directly from the integrated rates or shapes of the desorption peaks. ${ }^{57,58}$ Activation energies of desorption can be found from the appropriate Arrhenius plots or from the temperature relationship of the desorption paths, assuming constant preexponentials for the rate constants. Ehrlich ${ }^{57}$ was first to make quantitative measurements using fast heating with temperatures inversely proportional to times of desorption. Redhead ${ }^{58}$ introduced simplification in analysis and slow heating with linear temperatureversus-time relationships, which produced a spectra of desorption peaks from adsorption states of different binding energies.

If desorption can be neglected during adsorption studies, the sticking coefficient, $s$, at a given temperature can be obtained from the pressure change. ${ }^{58}$ Uncertainties in the true area, end effects of the filament, and wall effects tend to limit the accuracy obtainable. Nevertheless, flash desorption is the best presently available method of determining absolute surface coverage.

The greatest success of flash desorption has been the demon stration of discrete binding states or energies and the measurement of their abundances through the resolution of desorption spectra. To resolvediscrete binding states, differences in binding energies are necessary where there is little variation in the preexponential terms of the rate constants. Toresolve states close in energy, the absence of surface equilibrium and rapid inter conversion must also be invoked. Ehrlich ${ }^{59}$ showed that the absence of sur face equilibrium can be accounted for if states are associated with different crystallographic regions of the substrate, whose size precludes equilibration by surface diffusion in the desorption time.

Although desorption energies have been equated with binding stätës, chemically different states can still have sufficiently similar binding 
energies to require differentiation by means other than desorption. Thus, Bell and Gomer ${ }^{60}$ readily distinguished electropositive alpha and electronegative virgin states of $\mathrm{CO}$ by work-function measurements.

Kinetic studies of flash desorption are important for unraveling the conversion from one binding state to another and can show whether a given state corresponds to atomic or molecular adsorption. However, the pitfalls are many and varied. Desorption of undissociated diatomic molecules and molecular desorption from a dissociated but immobile layer by desorption of neighboring atom pairs both give rise to first-order kinetics. Also, since adsorption may be molecular at low temperatures, it may lead to activated dissociation as the temperature is raised, and finally to molecular evaporation, by association, with second-order kinetics. Similar considerations apply to isotope mixing studies carried out by combining flash desorption and mass spectrometry. The absence of mixing is sufficient evidence for nondissociative adsorption; however, mixing is not proof of dissociation. Thus the beta state of $\mathrm{CO}$ on tungsten undergoes isotopic exchange by local interaction of neighboring molecules, ${ }^{61}$ whereas kinetic and field-emission evidence suggest that truly random dissociation does not occur.

The analysis of integrated-rate or instantaneous-rate data can also be complex and ambiguous for other reasons. The shape of the desorption peaks is affected by possible variations in activation energy with coverage, by variations in the preexponential factor with coverage and temperature, and by surface heterogeneity of polycrystalline substrates. This can lead to a variation of apparent desorption energy or apparent reaction order with coverage. Complex interactions may, occur, involving rearrangement of substrate atoms, catalyzed desorption by interaction of an atom with a molecule in a specific configuration, or desorption through or accompanied by interconversion from one adsorbed state to another.

\section{Summary}

Recent advances in experimental techniques and in the understanding of the fundamental properties of gas-solid interactions have underscored the problems faced by early workers in surface-ionization measurements. More than one research specialty (field emission, flash desorption, mass spectrometry, low-energy electron diffraction, etc.) must be invoked to provide a complete picture of gas-solid phenomena. At present, many details of atomic processes on solid surfaces are unknown.

For a metal surface, different crystal planes possess different work functions giving a patch character to polycrystalline metal filaments. Adsorption of gases increases or decreases the work function, depending on the type of dipole layer formed. Both the ionization efficiency of the metal surface and the mean residence time of the species adsorbed are affected. 
Surface migration studies provide a map of the potential field acting on the adsorbed entities. The results show that binding is not localized at discrete sites when the surface temperature is high enough for rapid desorption to take place. At lower temperatures, flash-desorption studies show that molecular gases are chemisorbed on a metal surface in a multiplicity of states with discrete binding energies.

The chemical nature and structure of the particle being adsorbed profoundly affects the type of binding occurring between the gas and the substrate. Gomer ${ }^{62}$ has examined the adsorption of potassium and of carbon monoxide on tungsten for the purpose of defining the similarities and differences between metallic and covalent binding on the same substrate.

Field-emission studies yielded work-function changes with potassium atom density on individual crystal planes. ${ }^{63-65}$ Measurements were carried out for immobile adsorption with the substrate at $78^{\circ} \mathrm{K}$ during and after adsorption and after thermal equilibrium. The study of the adsorption of carbon monoxide on tungsten by flash-filament desorption, 59,61,66-70 field emission, ${ }^{33,60,71,72}$ electron-impact desorption, ${ }^{72}$ and low-energy electron diffraction ${ }^{73}$ reveals at least three types of binding states, which differ in their heats of adsorption, in electron-desorption cross sections, and in the sign of their dipole moments. The strongest bond state has a number of substates differing from each other by about $20 \mathrm{kcal} / \mathrm{mole}{ }^{4}$

Strong chemisorption of molecules with high ionization potentials is markedly different from metallic adsorption. Covalent adsorption shows definite discontinuous change in adsorption type, which can be correlated with differences in geometry of substrate structure and binding mode, but is less sensitive to the work function. Metallic adsorption shows no distinctly different adsorption states, but quantitative variations arise from differences in substrate geometry.and work function. The absence of separate states is more likely a consequence of rapid equilibrium of potassium over the surface and its continuous adaptation to a changing chemical environment with increased adsorption, than to a qualitative difference in the dependence of bond strength on structure between metallic and covalent adsorption. In both cases, however, substrate-adsorbate geometry appears to be important.

A rationale for these differences and similarities has been advanced by Gomer. ${ }^{62}$ In metallic adsorption, the relevant energy levels lie within the conduction band of the total system, with a large number of levels and many electrons participating in the binding. For the electronegative adsorption of carbon monoxide on tungsten, the system states involved may lie below the conduction band, using interband surface states of the substrate. Since these states are highly localized, the number of electrons involved in adsorption is small. Hence, the adsorption complex has a closer resemblence to a conventional chemical compound than found 
for metallic adsorption. Such surface compounds will be more sharply defined, with discontinuous changes as the configurations change.

Since the number of electrons involved in bonding is small, the net electron transfer involved and the polarizability of the adsorption complex will be small. The large differences in the ionization potential of carbon monoxide and the work functions of tungsten-crystal planes rule out electropositive polar binding. Very polar electronegative binding also is precluded, due to the relatively low electron affinity of the adsorbate.

Molecules of intermediate ionization potentials such as the alkali halides have not been investigated by these techniques at surface temperatures below that at which dissociation occurs. One aim of this study is to observe such interactions for lithium salts on rhenium surfaces using surface-ionization and flash-desorption methods. 


\section{CHAPTER III}

\section{THEORY OF SURFACE IONIZATION}

The theoretical description for ionization at hot metal surfaces by Langmuir and Kingdon ${ }^{74}$ quantitatively explains many experiments. The principal assumption is that of thermodynamic equilibrium, requiring uniform temperature, uniform work function, pressure equalization, and chemical equilibrium within the system.

In mass-spectrometer surface-ionization sources, the conditions necessary to achieve thermodynamic equilibrium are not fulfilled. The temperature and work functions of the various metal parts belonging to the system differ-considerably. Atoms and ions are separated due to application of external electric fields. Statistical derivations of the equation for surface ionization, in which the temperature dependence of the desorption probabilities for atoms and ions and the charge-transfer probabilities are considered separately, are better suited for a theoretical description of the surface-ionization process ${ }^{2,14,17,41,44,49,75}$

If the mean residence times of atoms and ions is great with respect to the time required for the thermal exchange of adsorbed particles with the surface and is great with respect to atom-ion charge transfer times, there exists a thermodynamic quasi-equilibrium within the adsorbed phase. The statistical theory then leads to the same results as the Saha-Langmuir theory.

Deviations from the fundamental Saha-Langmuir theory, such as the temperature dependence of the work function, ${ }^{14,76-80}$ the occurrence of excited states of atoms and ions, ${ }^{81,82}$ the reflections of atoms and ions from the surface, ${ }^{11,25,23}$ and the influence of external electric fields (Schottky effect): ${ }^{83}$ have been reviewed by Kaminsky ${ }^{2}$ and are not significant in the present study.

The influence of physical, chemical, and induced heterogeneities on the work function, and hence on the degree of surface ionization, is reviewed in Section II.B.1. Deviations from the assumptions of the Saha-Langmuir theory for ionization at polycrystalline surfaces of nonuniform work functions ${ }^{21,84-86}$ are'considered in Section III.F. Usually one retains the SahaLangmuir equation and considers the effect of the crystal structure by introducing an "effective" work function.

Deviations from the Saha-Langmuir equation due to the adsorption of foreign species on the hot metal surface are twofold. The physical character of the surface and principally the work function ${ }^{6 \hat{8}, 87-91}$ are changed by adsorbed layers. On the other hand, a chemical reaction can take place between the adsorbed foreign species and the atoms to be ionized.6,7,92 The adsorbed foreign species can originate from the residual gas in the system, condensation of the impinging particles, and/or diffusion of particles from the interior of the metal to its surface. 
The present study investigates ion emission with respect to the influence of adsorption of residual gases, the formation of condensed layers of the impinging beam material, and possible chemical reactions of the anionic constituent in the impinging beam with the hot surface.

A. The Saha-Langmuir Equation

The two quantities of interest for describing surface ionization are the degree of ionization,

$$
\alpha_{L}=\frac{Z_{+}}{Z_{0}},
$$

and the ionization coefficient,

$$
\beta_{L}=\frac{Z_{+}}{Z}
$$

Here, $Z_{+}$is the number of positive ions leaving the hot filament per unit area per unit time, $Z_{0}$ is the flux of neutral atoms from the same surface, and $Z$ is the flux of atoms impinging on the surface. In the steady-state condition, the fluxes are related by $Z=Z_{+}+Z_{0}$; hence,

$$
\beta_{L}=\frac{Z_{+}}{Z_{+}+Z_{0}}=\frac{\alpha_{L}}{l+\alpha_{L}} \text {. }
$$

Langmuir and Kingdon, ${ }^{18,93}$ using Saha's result, ${ }^{94}$ derived the temperature dependence of the degree of ionization, $\alpha_{L}$, under the simplifying assumption of thermodynamic equilibrium:

$$
\alpha_{L}=\frac{g_{+}}{g_{0}} \exp \frac{\phi-I}{k T}
$$

where $g_{+} / g_{0}$ is the ratio of the statistical weights of the ionic and atomic states, $\phi$ is the electron work function of the metal surface, $I$ is the ionization potential of the atom, $k$ is Boltzmann's constant, and $T$ is the absolute temperature.

Equation 3.4 actually is valid only for the ratio of the particle densities $n_{+} / n_{0}$ that deviates insignificantly from the fluxes by

$$
\alpha_{L}=\frac{z_{+}}{z_{0}}=\frac{n_{+}}{n_{0}} \sqrt{\frac{m_{0}}{m_{+}}}
$$

since the masses $m_{0}$ and $m_{+}$differ insignificantly. 
It may readily be appreciated that for $I .>\phi$ (e.g., copper. on tungsten), $\alpha_{\mathrm{L}}<1$, hence $\beta_{\mathrm{L}} \cong \alpha_{\mathrm{L}}$; for $\mathrm{I}<\phi$ (e.g.; cesium on tungsten), $\alpha_{\mathrm{L}}>1, \cdots$ hence $\beta_{L} \cong 1$; for $I \cong \phi, \alpha_{L}=g_{+} / g_{0}$, hence $\beta_{L}=g_{+} /\left(g_{+}+g_{0}\right)$.

\section{B. Sketch of Statistical Derivation*}

The degree of ionization $\alpha$, may be considered to be the product of two probabilities; that is,

$$
\alpha=\mathrm{W}_{1}(\mathrm{r}) \mathrm{W}_{2}(\mathrm{r})
$$

where $W_{l}(r)$ is the ratio of charge-transfer probabilities of converting an adatom into an ion or neutral atom at a distance $r$ from the metal surface, and $W_{2}(r)$ is the ratio of the desorption probabilities of ions and atoms.

Figure 3 shows a theoretical model similar to that used by others. ${ }^{2,49,75,95,96}$ In curve 1 , the distance $r$ of the ion is varied with the electron at $\mathbf{r} \rightarrow \infty$. In curve 2 , the distance $r$ of the electron is varied with the ion at $r=r_{0} ;(a)$ the electron passes the ion, (b) the electron passes through the center of the adion. In curve 3 , the distance $r$ of the adatom is varied.

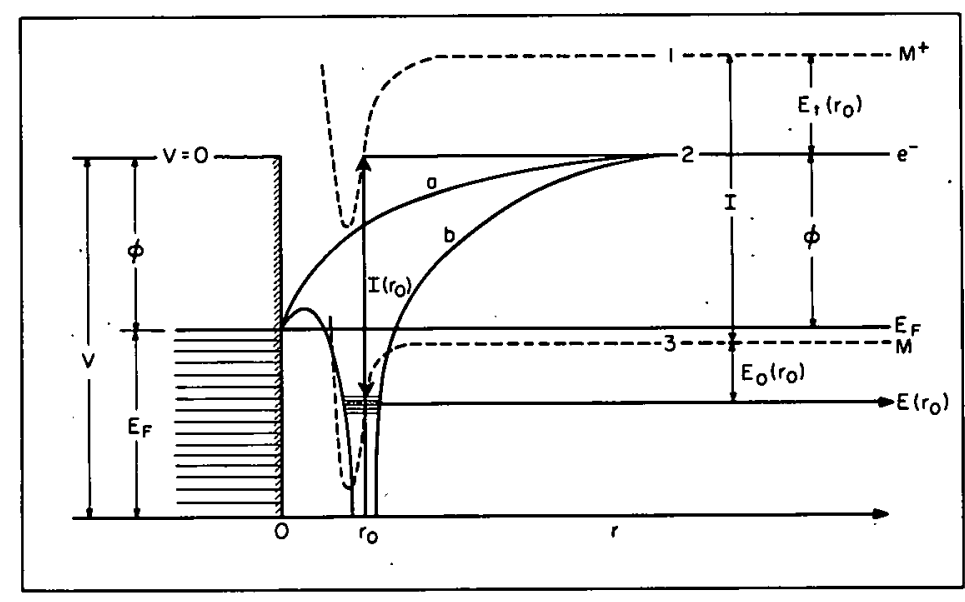

Fig. 3

Potential Energy of System Metal + Ion + Electron (symbols described in text)

$121-5490$

When a free atom approaches a metal surface, the re is a perturbation of the discrete energy levels of its external electrons. As a result of this perturbation, the allowed states are spread out into an energy band whose maximum $I\left(r_{0}\right)$ is shifted away from the energy level $E$ for the valence electron of the free atom at an infinite distance from the surface. This splitting is greater for smaller values of the distance of closest approach $r_{\text {min }}$ and for lower values of the ionization potential $I$ of the free atom. Above the critical distance $r_{0}$, electron exchange is no longer possible because the potential barrier between the adatom and the metal surface becomes broader and higher.

${ }^{*}$ The formulation of Weiershausen ${ }^{7} 5$ is used. 
When $r<r_{0}$, atomic andionic states of the adatom cannot be distinguished since the electron exchange is so intense. For $r>r_{0}$, the charge of the adatom is zero if the electron is in a state of energy $E=E\left(r_{0}\right)$ (the adatom is neutralized), or the charge is positive if the state $E=E_{+}\left(r_{0}\right)$ is not occupied by an electron.

Another way of saying this is that if the ionization energy, $I$, is given to an atom $M$ at a distance far from the surface, the system $M^{+}$plus its electron is raised to the level of curve l. If the electron is now placed in the Fermi sea of the metal, then the ion level will drop by an energy $\phi$. The ion is moved toward the surface, and the ion-surface potential will be in the vicinity of that of the adatom, shown by curve 3 . Since there are a number of unfilled levels in the metal, however, the electron may also be placed in a number of discrete states ranging from the vacuum level down to $\phi$ electron volts below the vacuum level. This will produce a band of ion states ranging from curve 1 to below curve 3. Transitions between ion and atom states are then possible at points where the potential curves cross. From Fig. 3, there are so many crossing points that there is access to and from any ion-atom level at the surface. The actual potential configurations and the relative positions of the potential minima are not known.

From Fermi statistics, the probability that the level $E=E\left(r_{0}\right)$ is occupied by an electron is

$$
W_{0}=W\left(E_{r_{0}}\right)=\left(1+\frac{g_{+}}{g_{0}} \exp \frac{E_{r_{0}}-E_{F}}{k T}\right)^{-1} .
$$

The probability that this level is not occupied by an electron is

$$
W_{+}=1-W\left(E_{r_{0}}\right)=\left[1+\frac{g_{0}}{g_{+}} \exp \frac{-\left(E_{r_{0}}-E_{F}\right)}{k T}\right]^{-1} .
$$

The symbol $E_{F}$ is the energy of the Fermi level or the electrochemical potential of the metal. The Fermi level is defined at a temperature of $0^{\circ} \mathrm{K}$ as the level below which all energy states are occupied by electrons and above which all energy states are unoccupied.

The charge-transfer probability of the adatom is then defined by

$$
W_{1}(\dot{r})=\frac{W_{+}}{W_{0}}=\frac{g_{+}}{g_{0}} \exp \frac{E_{r_{0}}-E_{F}}{k T} .
$$

The difference, $E_{r_{0}}-E_{F}$, may be seen from Fig. 3 to be valid for an arbitrary $\mathrm{r}_{0}$ even when $\phi>\mathrm{I}$;

$$
E\left(r_{0}\right)-E_{F}=\phi-I\left(r_{0}\right)=\Delta Q=E_{+}\left(r_{0}\right)-E_{0}\left(r_{0}\right)+\phi-I \text {, }
$$


where $I\left(r_{0}\right)$ is the ionization potential for the adatom at a distance $r_{0}$, and $\Delta Q$ is a symbol representing the difference $E\left(r_{0}\right)-E_{F}$. Note that $I$ is the only quantity that is independent of the substrate. Equation 3.9 may now be written as

$$
W_{1}(r)=\frac{W_{+}}{W_{0}}=\frac{g_{+}}{g_{0}} \exp \frac{E_{+}-E_{0}+\phi-I}{k T}
$$

The ratio of the desorption probabilities $\mathrm{P}_{+}$and $\mathrm{P}_{0}$ of ions and atoms from a surface is

$$
W_{2}(r)=\frac{P_{+}(r)}{P_{0}(r)}
$$

The probability that the kinetic energy of a given atom or ion will exceed the desorption energy $E_{+}$or $E_{0}$ is, according to Frenkel, ${ }^{97}$

$$
\mathrm{P}_{+}(\mathrm{r})=\nu_{+}^{0} \exp \frac{-\mathrm{E}_{+}}{\mathrm{kT}}=\frac{1}{\tau_{+}}
$$

and

$$
P_{0}(r)=\nu_{0}^{0} \exp \frac{-E_{0}}{k T}=\frac{1}{\tau_{0}}
$$

where $\nu_{+}^{0}$ and $\nu_{0}^{0}$ are slightly temperature-dependent frequency factors. For nonactivated adsorption, the mean residence times $\tau_{+}$and $\tau_{0}$ of the adsorbed particles on the surface are inversely proportional to their desorption probabilities.

According to Eqs. 3.6-3.14, the degree of ionization $\alpha$ is obtained as

$$
\alpha=\frac{g_{+} \nu_{+}^{0}}{g_{0} \ddot{\nu}_{0}^{0}} \exp \frac{\phi-I}{\mathrm{kT}}
$$

and is seen to be independent of the distance $r$. Except for the factor $\nu_{+}^{0} / \nu_{0}^{0}$, approximately of the order of 1 , the same result is obtained for $\alpha$ as that derived by Langmuir from thermodynamic equilibrium considerations.

\section{Effect of Mean Residence Times}

The statistical derivation of the Saha-Langmuir equation given above rests mainly on two assumptions: (1) The adsorption lifetimes are large with respect to times required for electron transfer between metal and atom, and (2) the adsorption lifetimes (of atoms and ions) are sufficient for complete temperature equilibrium with the metal surface. When these assumptions 
are no longer valid: deviations from the Saha-Langmuir equation take place. To show this, consider the changes taking place in the surface concentration of the species with both atoms and ions impinging on the surface.

With the assumption of a zero reflection coefficient, the steady-state concentration changes of adions, $n_{+}$, and adatoms, $n_{0}$, may be written as

$$
\frac{d n_{t}}{d t}=Z_{+}^{0}+P_{a i} n_{0}-P_{+} n_{+}-P_{i a} n_{+}=0
$$

and

$$
\frac{d n_{0}}{d t}=Z_{0}^{0}+P_{i a} n_{+}-P_{0} n_{0}-P_{a i} n_{0}=0
$$

The terms $Z_{+}^{0}$ and $z_{0}^{0}$ represent the number of ions and atoms, respectively, impinging on the surface per unit time per unit area from the gas phase. The terms $P_{+} n_{+}$and $P_{0} n_{0}$ are the current densities of the desorbed.ions $Z_{+}$ and of the desorbed atoms $Z_{0}$, respectively. Since these terms are equal to the time rate of change of their surface concentrations by desorption, the defining equations are

$$
Z_{+}=\left(\frac{d n_{t}}{d t}\right)_{d}=P_{+} n_{+}: Z_{0}=\left(\frac{d n_{0}}{d t}\right)_{d}=P_{0} n_{0} .
$$

The transition probabilities for atom-ion interchange at the surface likewise depend on the initial surface concentrations of the species and are defined by

$$
\left(\frac{d n_{t}}{d t}\right)_{i a}=P_{i a} n_{+} ;\left(\frac{d n_{0}}{d t}\right)_{a i}=P_{a i} n_{0},
$$

where the indexes ia and ai denote the transition ion $\rightarrow$ atom and atom $\rightarrow$ ion, respectively.

By suitable manipulation of Eqs. 3.16 and 3.17 , the ratio of surface concentration of ions and atoms is found to be

$$
\frac{n_{+}}{n_{0}}=\frac{\left(P_{0}+P_{a i}\right) Z_{+}^{0}+P_{a i} Z_{0}^{0}}{\left(P_{+}+P_{i a}\right) Z_{0}^{0}+P_{i a} Z_{+}^{0}}
$$

Two situations (thermodynamic equilibrium and steady state) are considered for their effect on the degree of ionization $\alpha$. 
1. Thermodynamic Equilibrium

Here

$$
z_{+}^{0}=\alpha_{L} z_{0}^{0}
$$

and

$$
\frac{Z_{+}}{Z_{0}}=\alpha_{L}=\frac{n_{+} P_{+}}{n_{0} P_{0}} ; \quad P_{0}=\frac{n_{+} P_{+}}{n_{0} \alpha_{L}} .
$$

Substituting Eqs. 3.21 and 3.22 into Eq. 3.20 and rearranging produces

$$
\frac{\mathrm{n}_{+}}{\mathrm{n}_{0}}=\frac{\mathrm{P}_{\mathrm{ai}}}{\mathrm{Pia}}
$$

which says that the ratio of surface concentrations of adions to adatoms is proportional to the ratio of the transition probabilities. Hence, the degree of surface ionization,

$$
\alpha=\alpha_{L}=\frac{n_{+} P_{+}}{n_{0} P_{0}}=\frac{P_{a i} P_{+}}{P_{i a} P_{0}},
$$

is given as the product of the transition probability ratio and the desorption probability ratio.

\section{Steady State}

When only atoms impinge on the surface, $Z_{+}^{0}=0$. From Eq. 3.20 it follows that

$$
\frac{\mathrm{n}_{+}}{\mathrm{n}_{0}}=\frac{\mathrm{P}_{\mathrm{ai}}}{\mathrm{P}_{\mathrm{ia}}+\mathrm{P}_{+}}
$$

and the ratio of the surface concentrations is no longer proportional to the transition probabilities. From Eq. 3.16,

$$
\alpha=\frac{Z_{+}}{Z_{0}}=\frac{n_{+} P_{+}}{n_{0} P_{0}}=\frac{P_{a i} P_{+}}{P_{i a} P_{0}} \cdot \frac{1}{1+P_{+} / P_{i a}} .
$$

For complete temperature equilibrium of the adsorbed species with the surface, Eq. 3.24 may be used giving

$$
\alpha=\alpha_{L} \frac{1}{1+P_{+} / P_{i a}}
$$


Only when the vaporization probability per unit time of ions, $P_{+}$, is small with respect to the transition probability of ions $\rightarrow$ atoms, $P_{i a}$, is $c=\alpha_{L}$.

D. Influence of Anionic Constituents in the Molecular Beam

When molecular beams.impinge on a hot metal surface, dissociation and recombination of the various species must be considered. Equations for the general case of surface ionization of diatomic molecules (specifically, the alkali halides) will be derived and may be simplified under certain assumptions.*

Let MX, a beam of molecules or the vapor species in a gas, strike or fall on a hot metal surface. Emission fluxes of the particles $X, X^{-}, X_{2}$, $M, M^{+}$, and $M X$ with flux densities of $Z_{X}, Z_{X}{ }^{-}=\gamma Z_{X}, Z_{X_{2}}, Z_{M}, Z_{M^{+}}=0, Z_{M}$. and $Z_{M X}$ are assumed, where $\alpha$ and $\gamma$ are the degrees of surface ionization of the species involved. Under the steady-state conditions, where $Z$ is the initial rate of impingement of $\mathrm{MX}$ molecules in molecules $\mathrm{cm}^{-2} \mathrm{sec}^{-1}$, conservation of flux particles gives

$$
\mathrm{Z}=\mathrm{Z}_{\mathrm{MX}}+\mathrm{z}_{\mathrm{M}^{+}}+\mathrm{z}_{\mathrm{M}^{+}}=\mathrm{z}_{\mathrm{MX}}+\mathrm{z}_{\mathrm{M}^{(1+\alpha)}}
$$

and

$$
\mathrm{Z}=\mathrm{z}_{\mathrm{MX}}+\mathrm{z}_{\mathrm{X}}+\mathrm{z}_{\mathrm{X}}{ }^{-}+2 \mathrm{Z}_{\mathrm{X}_{2}}=\mathrm{Z}_{\mathrm{MX}}+\mathrm{z}_{\mathrm{X}}(1+\gamma)+2 \mathrm{Z}_{\mathrm{X}_{\tilde{\mathrm{z}}}}
$$

The conservation of species on the surface in the steady-state condition is given by

$$
Z-Z_{M X}=k_{1} n_{M X}-k_{1}^{i} n_{M} n_{X}
$$

and

$$
k_{2} n_{X}^{2}=k_{2}^{\prime} n_{X_{2}}+z_{X_{2}}
$$

where $k_{1}$ and $k_{1}^{\prime}$ are the rate constants for dissociation and recombination of molecules $\mathrm{MX}$ on the surface, and $k_{2}$ and $k_{2}^{\prime}$ are the rate constants for the dissociation and recombination of $X_{2}$ on the surface. The surface concentration, $n_{i}$, of the $i$ th species is related to the flux $Z_{i}$ by

$$
\mathrm{Z}_{\mathrm{i}}=\mathrm{P}_{\mathrm{i}} \mathrm{n}_{\mathrm{i}}
$$

\footnotetext{
$*_{\text {An extension of the work of Potekhina }} 98$ is involved.
} 
where

$$
\mathrm{P}_{i}=\mathrm{P}_{\mathrm{i}}^{0} \exp \left(-\frac{\lambda_{\mathrm{i}}}{\mathrm{kT}}\right)=\nu_{\mathrm{i}}^{0} \exp \left(-\frac{\lambda_{\mathrm{i}}}{\mathrm{kT}}\right)
$$

$\lambda_{i}$ being the desorption activation energy for the ith species.

From Eqs. 3.28-3.31, the following equation results:

$$
Z_{M}(1+\alpha)=k_{1} n_{M X}-k_{1}^{\prime} n_{M} n_{X}^{\prime}=k_{1} \frac{Z_{M X}}{P_{M X}}-k_{1}^{\prime} \frac{Z_{M} Z_{X}}{P_{M} P_{X}} .
$$

Substituting $Z_{M X}=Z-Z_{M}(1+\alpha)$ in Eq. 3.34 gives

$$
z_{M}(1+\alpha)=\frac{k_{1}}{P_{M X}} z-\frac{k_{1}}{P_{M X}} z_{M}(1+\alpha)-k_{1}^{\prime} \frac{z_{M} Z_{X}}{P_{M} P_{X}}
$$

or

$$
\mathrm{Z}_{M}(1+\alpha)\left(1+\frac{\mathrm{P}_{M X}}{k_{1}}\right)+\frac{k_{1}^{\prime} \mathrm{P}_{M X} \mathrm{Z}_{M} \mathrm{Z}_{X}}{\mathrm{k}_{1} \mathrm{P}_{M} \mathrm{P}_{\mathrm{X}}}=\mathrm{Z}
$$

Let

$$
x_{1}=\frac{k_{1}^{\prime} P_{M X}}{k_{1} P_{M}{ }^{P} X}
$$

On substitution of this relationship, Eq. 3.35 then becomes

$$
\mathrm{Z}_{\mathrm{M}}(1+\alpha)\left(1+\frac{\mathrm{P}_{\mathrm{MX}}}{\mathrm{k}_{1}}\right)+\mathrm{x}_{1} \mathrm{Z}_{\mathrm{M}} \mathrm{Z}_{\mathrm{X}}=\mathrm{Z}
$$

Inspection of Eqs. 3.28 and 3.29 shows that

$$
Z_{M}(1+\alpha)=Z_{X}(1+\gamma)+2 Z_{X_{2}}
$$

Using Eq. 3.32 in Eq. 3.31 produces the following expression for $\mathrm{Z}_{\mathrm{X}_{2}}$ :

$$
\mathrm{Z}_{\mathrm{X}_{2}}=\frac{\mathrm{k}_{2} \mathrm{Z}_{\mathrm{X}}^{2}}{\mathrm{P}_{\mathrm{X}}^{2}\left(1+\frac{\mathrm{k}_{2}^{\prime}}{\mathrm{P}_{\mathrm{X}_{2}}}\right)}:=\frac{\mathrm{k}_{2} \mathrm{P}_{\mathrm{X}_{2}} \mathrm{Z}_{\mathrm{X}}^{2}}{\mathrm{P}_{\mathrm{X}}^{2} \mathrm{k}_{2}^{\prime}\left(1+\frac{\mathrm{P}_{\mathrm{X}_{2}}}{\mathrm{k}_{2}^{\prime}}\right)}=\mathrm{x}_{2} \mathrm{Z}_{\mathrm{X}}^{2},
$$


where

$$
x_{2}=\frac{k_{2} P_{X_{2}}}{P_{X}^{2} k_{2}^{\prime}\left(1+\frac{P_{X_{2}}}{k_{2}^{\prime}}\right)}
$$

On substitution of $\mathrm{Z}_{\mathrm{X}_{2}}$ from Eq. 3.39, Eq. 3.38 becomes

$$
\mathrm{Z}_{\mathrm{M}}(1+\alpha)=\mathrm{Z}_{\mathrm{X}}\left(1+\gamma+2 \mathrm{x}_{2} \mathrm{Z}_{\mathrm{X}}\right)
$$

or

$$
Z_{X}=\frac{Z_{M}(1+\alpha)}{1+\gamma+2 x_{2} z_{X}}
$$

When Eq. 3.41 is substituted in Eq. 3.37 and $Z_{M}(1+\alpha)$ is factored out, the result is

$$
Z_{M}(1+\alpha)\left(1+\frac{P_{M X}}{k_{1}}+\frac{x_{1} Z_{M}}{1+\gamma+2 x_{2} Z_{X}}\right)=Z \text {. }
$$

Equation 3.42 can be used to determine the behavior of the flux $Z_{M}$ at different surface temperatures. In the subsequent experiments, it is more convenient to determine the behavior of the molecular ionization coefficient, $\beta_{\mathrm{MX}}$, as a function of the temperature and the intensity of the incident beam of $\mathrm{MX}$ molecules. By definition, $\beta_{\mathrm{MX}}=\mathrm{Z}_{\mathrm{M}^{+}} / \mathrm{Z}=\alpha \mathrm{Z}_{\mathrm{M}} / \mathrm{Z}$.

Substitution of this expression for $Z_{M}$ into Eq. 3.42 yields

$$
1+\frac{P_{M X}}{k_{1}}+\frac{x_{1} z}{1+\gamma+2 x_{2} Z_{X}} \cdot \frac{\beta_{M X}}{\alpha}=\frac{\alpha}{(1+\alpha) \beta_{M X}}
$$

Equation 3.43 is the primary equation under consideration. The effect of temperature on $x_{1}$ and $x_{2}$ and simplifying conditions are amplified in the following paragraphs.

The emitter surface-temperature dependence of $x_{1}$ can be illustrated as follows: Assume that the rate constants $k_{i}$ and $k_{i}^{\prime}$ are related to the activation energies for dissociation and recombination at the surface by Arrhenius-type equations; i.e.,

$$
\mathrm{k}_{1}=\dot{\mathrm{P}}_{1} \exp \left(-\ell_{1} / \mathrm{kT}\right), \quad \mathrm{k}_{1}^{\prime}=\mathrm{P}_{1}^{\prime} \exp \left(-\ell_{1}^{\prime} / \mathrm{kT}\right)
$$

and that the difference in the activation energies, $\ell_{1}-\ell_{1}$, determines the heat of dissociation at the surface, $D(s)$. Then using the cyclic process gives 


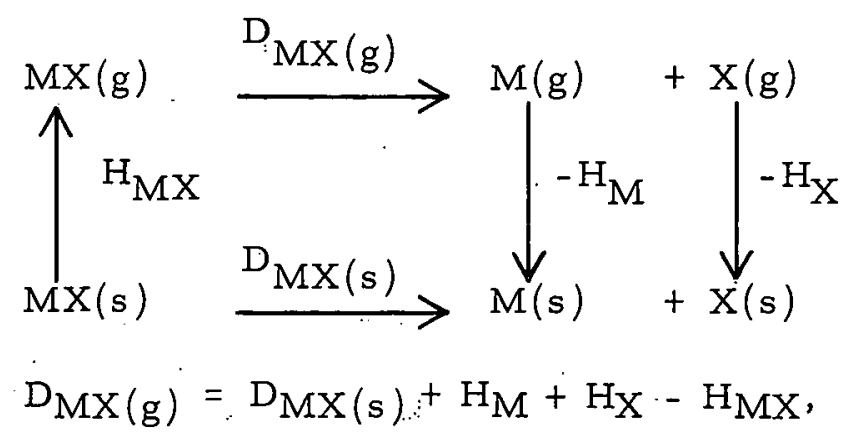

where $\mathrm{H}_{i}$ is the heat of desorption of the species $i$. If $\lambda_{i}=H_{i}$, i.e., if the desorption activation energy, $\lambda_{i}$, (from Eq. 3.33) is equal to the heat of desorption, $\mathrm{H}_{\mathbf{i}}, \mathrm{Eq} .3 .36$ becomes

$$
\begin{aligned}
\mathrm{x}_{1} & =\frac{\mathrm{P}_{1}^{\prime} \mathrm{P}_{M X}^{0}}{\mathrm{P}_{1} \mathrm{P}_{M}^{0} \mathrm{P}_{\mathrm{X}}^{0}} \exp \frac{\ell_{1}-\ell_{1}^{\prime}-\mathrm{H}_{\mathrm{MX}}+\mathrm{H}_{\mathrm{M}}+\mathrm{H}_{\mathrm{X}}}{\mathrm{kT}} \\
& =\frac{\mathrm{P}_{1}^{\prime} \nu_{M X}^{0}}{\mathrm{P}_{1} \nu_{M}^{0}{ }^{\nu}} \exp \frac{\mathrm{D}_{\mathrm{MX}}(\mathrm{g})}{\mathrm{kT}}=\mathrm{C}_{1} \exp \frac{\mathrm{D}_{\mathrm{MX}(\mathrm{g})}}{\mathrm{kT}}
\end{aligned}
$$

Similarly, Eq. 3.40 becomes

$$
\begin{aligned}
\mathrm{x}_{2} & =\frac{\mathrm{P}_{2} \mathrm{P}_{\mathrm{X}_{2}}^{0}}{\mathrm{P}_{2}^{\prime} \mathrm{P}^{0^{2}}\left(1+\frac{\mathrm{PX}_{2}}{\mathrm{k}_{2}^{\prime}}\right)} \exp \frac{\ell_{2}^{\prime}-\ell_{2}-\mathrm{H}_{\mathrm{X}_{2}}+2 \mathrm{H}_{\mathrm{X}}}{\mathrm{kT}} \\
& =\frac{\mathrm{P}_{2} \nu_{\mathrm{X}_{2}}^{0}}{\mathrm{P}_{2}^{\prime} \nu_{\mathrm{X}}^{0^{2}}}\left(1+\frac{\mathrm{P}_{\mathrm{X}_{2}}}{\mathrm{k}_{2}^{\prime}}\right)^{-1} \exp \frac{\mathrm{D}_{\mathrm{X}_{2}(\mathrm{~g})}}{\mathrm{kT}} \\
& =\mathrm{C}_{2}\left(1+\frac{\mathrm{P}_{\mathrm{X}_{2}}}{\mathrm{k}_{2}^{\prime}}\right)^{-1} \exp \frac{\mathrm{D}_{\mathrm{X}_{2}(\mathrm{~g})}}{\mathrm{kT}}
\end{aligned}
$$

In Section III.D.2, a method is presented for the experimental determination of $D_{\mathrm{MX}}(\mathrm{g})$ by surface-ionization measurements.

The primary equation (Eq. 3.43) can be simplified in certain respects, depending on the experimental conditions used as well as the relative magnitudes of the different terms. The behavior of the degree of ionization $\dot{\gamma}=\mathrm{Z}_{\mathrm{X}^{-}} /\left(\mathrm{Z}_{\mathrm{X}}+\mathrm{Z}_{\mathrm{X}^{-}}\right)$as a function of temperature and its effect on the ionization coefficient $\beta_{M X}$ are first considered. The behavior of $\gamma$ is given by the Saha-Langmuir equation for negatively charged ions as 


$$
\gamma=\frac{g_{X}-}{G_{X}} \exp \frac{S_{X}-\phi}{k T}
$$

where $g_{X}-/ G_{X}$ is the ratio of the statistical weights of the ionic and atomic states. The temperature dependence of the statistical weight for neutral halogen species is given by

$$
G_{X}=4+2 \exp -\frac{E_{X}}{k T}
$$

where $E_{X}$ is the excitation energy for an excited state, and $S_{X}$ is the electron affinity of atom $X$. Of the halogens, chlorine has the greatest electron affinity: Bailey ${ }^{99}$ gives a value of $\mathrm{S}_{\mathrm{Cl}}=3.75 \mathrm{eV}$. Using $\phi=5.1 \mathrm{eV}$, and with the excitation energy to the first excited state given by $E_{C l}=882.5 \mathrm{~cm}^{-1}$ (JANAF Tables), ${ }^{100}$ produces the value of $\gamma$ versus temperature for chlorine in Table IV.

TABLE IV. Degree of Ionizàtion, $\gamma$, vs : Témperature. for Chlorine Atoms

\begin{tabular}{lcccc}
\hline $\mathrm{T},{ }^{\circ} \mathrm{K}$ & $\mathrm{G}_{\mathrm{Cl}}$ & $\frac{{ }_{\mathrm{S}}{ }-\phi}{\mathrm{kT}}$ & $\exp \frac{{ }^{S_{\mathrm{Cl}}-\phi}}{\mathrm{kT}}$ & $\gamma$ \\
\hline 1000 & 4.71 & -15.65 & $1.60 \times 10^{-7}$ & $3.40 \times 10^{-8}$ \\
1500 & 4.86 & -10.43 & $2.95 \times 10^{-5}$ & $6.07 \times 10^{-6}$ \\
2000 & 5.06 & -7.825 & $4.00 \times 10^{-4}$ & $7.91 \times 10^{-5}$ \\
2500 & 5.20 & -6.26 & $1.90 \times 10^{-3}$ & $3.65 \times 10^{-4}$ \\
\hline
\end{tabular}

At the highest temperature considered $\left(\mathrm{T}=2500^{\circ} \mathrm{K}\right)$, the degree of ionization $\gamma$ is less than $0.1 \%$ for chlorine, considerably less for other. halogens; and may be neglected in Eq. 3.43 when halogens are the anionic constituents.

It is also possible to show that where few $X_{2}$ molecules are produced, Eq. 3.39, $\mathrm{Z}_{\mathrm{X}_{2}}=\mathrm{x}_{2} \mathrm{Z}_{\mathrm{X}}^{2}$, requires that $\mathrm{x}_{2}<<1$. Consequently $2 \mathrm{x}_{2} \mathrm{Z}_{\mathrm{X}}<<1$. The presence of $X_{2}$ molecules can be determined experimentally by mass spectrometric measurements. The primary equation (Eq. 3.43) then becomes, on neglecting the terms $\gamma$ and $2 x_{2} Z_{X}$,

$$
1+\frac{P_{M X}}{k_{1}}+x_{1} z \frac{\beta_{M X}}{\alpha}=\frac{\alpha}{(1+\alpha) \beta_{M X}}
$$


In terms of the experimentally variable conditions of incident flux and emitter temperature, two situations of interest arise based on the magnitude of the molecular flux $\mathrm{Z}_{\mathrm{MX}}$ leaving the surface ionization filament.

$$
\text { 1. Complete Dissociation of } \mathrm{MX}\left(\dot{\mathrm{Z}}_{\mathrm{MX}}=0\right)
$$

For low incident-beam intensities, Z, or high temperatures, complete dissociation of molecules MX occurs at the surface-ionization filament. With this stipulation, comparison of Eqs. 3.28 and 3.42 shows that

$$
\frac{P_{M X}}{k_{1}}+\frac{x_{1} Z_{M}}{1+\gamma+2 x_{2} Z_{X}} \approx 0
$$

or, using the simplified expression,

$$
\frac{P_{M X}}{k_{1}}+\frac{x_{1} Z \beta M X}{\alpha} \approx 0
$$

Hence, the molecular-ionization coefficient is given by Eq. 3.49 as

$$
\beta_{M X}=\frac{\alpha}{1+\alpha}=\beta_{M}
$$

and is equal to the atomic-ionization coefficient.

Thus, only when the emitter temperature is sufficiently high or the salt vapor pressure sufficiently low is the ionization coefficient for molecules given by the Saha-Langmuir formula. The effect of the anionic constituent on surface ionization is then of no consequence in this situation.

\section{Incomplete Dissociation of $\mathrm{MX}\left(\mathrm{Z}_{\mathrm{MX}} \neq 0\right)$}

At high incident-beam intensities or lower temperatures, a significant fraction of MX molecules may be desorbed from the surface. The effect of the second and third terms in Eq. 3.49 on the ionization coefficient may then be determined as follows: Subtracting Eq. 3.49 from a similar equation with another incident-beam intensity, $Z_{2}$, eliminates the term $\mathrm{P}_{\mathrm{MX}} / \mathrm{k}_{1}$, giving

$$
x_{1}\left(\beta_{2} z_{2}-\beta_{1} z_{1}\right)=\frac{\alpha^{2}}{1+\alpha}\left(\frac{1}{\beta_{2}}-\frac{1}{\beta_{1}}\right),
$$

provided the degree of surface ionization $\alpha$ does not depend on the incidentbeam intensity. Since $Z_{2}=c Z_{1}, E q .3 .51$ reduces to 


$$
x_{1} z_{1}=\frac{\alpha^{2}}{1+\alpha} \cdot \frac{\beta_{1}-\beta_{2}}{\beta_{1} \beta_{2}} \cdot \frac{1}{c \beta_{2}-\beta_{1}}
$$

Since $x_{1}$ is given by Eq. 3.46 as

$$
x_{1}=C_{1} \exp \frac{D_{M X}(g)}{k T}
$$

the slope of the line of $\ln x_{1} Z_{1}$ versus $1 / k T$ should give directly $D_{M X}(g)$, the dissociation energy of $M X$. Should this be the case, the previous assumption that few $X_{2}$ molecules are formed is justified. Otherwise, $2 x_{2} Z_{X} \geq 1$ in Eq. 3.43 and cannot be neglected, and $\mathrm{D}_{\mathrm{MX}(\mathrm{g})}$ cannot be determined uniquely.

The magnitude of $P_{M X} / k_{1}$ may be determined by substituting $\mathrm{x}_{1} \mathrm{Z}_{1}$ of Eq. 3.52 back into Eq. 3.49 .

a. Extension of Derivation. It is instructive to look at the relative effects of the quantities $P_{M X} / k_{1}$ and $x_{1} z$ on the molecular-ionization coefficient, $\beta_{\mathrm{MX}}$. Solving for $\beta_{\mathrm{MX}}$ in Eq. 3.49 gives

$$
\beta_{\mathrm{MX}}=\frac{\alpha}{2 \mathrm{x}_{1} Z}\left(1+\frac{\mathrm{P}_{\mathrm{MX}}}{\mathrm{k}_{1}}\right)\left[\sqrt{1+\frac{4 \mathrm{x}_{1} \mathrm{Z}}{(1+\alpha)\left(1+\frac{\mathrm{P}_{\mathrm{MX}}}{\mathrm{k}_{1}}\right)^{2}}}-1\right]
$$

At low incident-beam intensities where $x_{1} Z$ is small, a series expansion of the radical leads to

$$
\beta_{\mathrm{MX}}=\frac{\alpha}{1+\alpha} \cdot \frac{1}{1+\frac{\mathrm{P}_{\mathrm{MX}}}{\mathrm{k}_{1}}}=\beta_{\mathrm{M}} \frac{1}{1+\frac{\mathrm{P}_{M X}}{\mathrm{k}_{1}}}
$$

where terms of higher order than the second have been neglected in the series expansion. Starodubtsev ${ }^{101}$ derived this expression directly by assuming no chemical equilibrium between the particles adsorbed on the surface and neglecting the reverse reaction of surface particle recombination; e.g., $k_{1}^{\prime} n_{M^{n}}^{n_{X}}=0$ in Eq. 3.30 .

At high temperatures, well above the threshold temperature, the surface concentration of molecules $M X$ is small, dissociation of molecules on the surface may be more probable than the evaporation of molecules, and the flux of particles desorbed from the surface consists mainly of atoms. Such a condition can exist when the desorption probability, $\mathrm{P}_{\mathrm{MX}}$, of molecules $\mathrm{MX}$ is small with respect to the rate of dissociation, $\mathrm{k}_{1}$, of molecules MX adsorbed on the surface. In other words, when the 
surface-dissociation activation energy is less than the desorption activation energy, $l_{1}-\lambda_{\mathrm{MX}}<0$; therefore, $\mathrm{P}_{\mathrm{MX}} / \mathrm{k}_{1}<<1$ and the molecular-ionization coefficient $\left(\beta_{\mathrm{MX}}=\beta_{\mathrm{M}}\right)$. (The preexponential term of $\mathrm{P}_{\mathrm{MX}} / \mathrm{k}_{\mathrm{I}}$ is small.)

Note, however, that Eq. 3.54 is also valid where $\ell_{1}-\lambda_{\mathrm{MX}}>$ $1 / 2 \mathrm{D}_{\mathrm{MX}}(\mathrm{g})$; the increase of the numerator under the radical sign in Eq. 3.53 does not keep up with the increase in the denominator on temperature reduction. As the temperature is reduced, the concentration of surface molecules is increased and desorption of $\mathrm{MX}$ molecules becomes more probable. Equation 3.54 again applies with $P_{M X} / k_{1}>>1$.

When $l_{1}-\lambda_{\mathrm{MX}}<1 / 2 D_{\mathrm{MX}}(\mathrm{g})$, the second term under the radical sign in Eq. 3.53 increases exponentially with temperature reduction due to the reduced rate of dissociation and recombination. Under these conditions, Eq. 3.53 can be simplified to

$$
\beta_{\mathrm{MX}}=\frac{\alpha}{1+\alpha} \sqrt{\frac{1+\alpha}{\mathrm{x}_{1} Z}}=\beta_{\mathrm{M}} \sqrt{\frac{1+\alpha}{\mathrm{x}_{1} Z}}
$$

and the dependence of $\mathrm{P}_{\mathrm{MX}} / \mathrm{k}_{\mathrm{I}}$ vanishes, even though $\mathrm{P}_{\mathrm{MX}} / \mathrm{k}_{1}$ may be greater than 1. Equation 3.55 may be derived by assuming a chemical equilibrium to exist in the adsorbed layer $\left(k_{1} n_{M X}=k_{1}^{\prime} n_{M} n_{X}\right)$, together with the existence of thermodynamic equilibrium between the adatoms and the surface. ${ }^{102}$

These results (Eqs. 3.50, 3.54, and 3.55) can describe the course of the surface-ionization reaction occurring on a hot rhenium surface when molecules MX are adsorbed. At very high temperatures and sufficiently low beam intensities of $\mathrm{MX}$ molecules, complete dissociation of $\mathrm{MX}$ occurs and Eq. 3.50 applies; that is, $\beta_{M X}=\beta_{M}$. As the surface temperature is reduced, surface coverage increases and recombination becomes possible. On further temperature reduction, emission of $\mathrm{MX}$ molecules may exceed dissociation. Under these conditions, $k_{1} n_{M X} \cong k_{1}^{\prime} n_{M} n_{X} \gg Z-Z_{M X}$, so that $Z \cong Z_{M X}$, and the ion flux $Z_{M}+$ approaches zero: Although an equilibrium of dissociation and recombination may exist on the surface at low temperatures, the rates for these processes becomes very small.

An additional factor not previously considered arises when there is the possibility of an appreciable chemical reaction other than just chemisorption between the anionic constituent of the molecular beam and the surface-ionization emitter. A typical example is the formation of gaseous tungsten trioxide when oxygen-containing compounds impinge on a hot tungsten filament. For a halogen atom-rhenium reaction, leading to monomeric diatomic gaseous rhenium halides, an additional conservation equation for species on the surface can be written as

$$
k_{3} n_{S} n_{X}=k_{3}^{i} n_{S X}+Z_{S X}
$$


where the indexes $S$ and SX stand for the substrate and substrate halide, respectively. The conservation of flux particles is now given by an extension to Eq. 3.29,

$$
\mathrm{Z}=\mathrm{Z}_{\mathrm{MX}}+\mathrm{Z}_{\mathrm{X}}(1+\gamma)+2 \mathrm{Z}_{\mathrm{X}_{2}}+\mathrm{Z}_{\mathrm{SX}}
$$

Proceeding as previously outlined,

$$
\mathrm{Z}_{\mathrm{SX}}=\mathrm{x}_{3} \mathrm{Z}_{\mathrm{X}}
$$

with

$$
x_{3}=\frac{k_{3} P_{X S} Z_{S}}{k_{3}^{\prime} P_{S} P_{X}\left(1+\frac{P_{S X}}{k_{3}^{\prime}}\right)} 。
$$

Equation 3.41 becomes

$$
Z_{X}=\frac{Z_{M}(1+\alpha)}{1+\gamma+2 x_{2} Z_{X}+x_{3}}
$$

leading to an extension of the primary equation (Eq. 3.43) as

$$
1+\frac{P_{M X}}{k_{1}}+\frac{x_{1} Z}{1+\gamma+2 x_{2} Z_{X}+x_{3}} \cdot \frac{\beta_{M X}}{\alpha}=\frac{\alpha}{(1+\alpha) \beta_{M X}}
$$

The formation of gaseous rhenium halides other than monomeric diatomic species results in a modification of $x_{3}$. The primary effect of rhenium halide formation would be to displace the surface equilibrium towards greater dissociation of MX molecules at a. given temperature. The presence of rhenium halides should be readily detected by mass-spectrometer methods.

b. Comparison with Experiment. Other experimental results on the surface ionization of salt molecules have a bearing on the previous discusisions. Zandberg and Tontegode ${ }^{8}$ investigated the temperature dependence of the surface-ionization currents of alkali atoms and alkali chloride molecules on polycrystalline rhenium wire. Above a certain temperature, the temperature dependence of the surface-ionization currents of lithium atoms and lithium chloride molecules became the same. The thresholdionization temperature for the lithium atomic beam was lower than that for the lithium chloride molecular beam; i.e., a large decrease in the lithium ion current from the molecular beam was found in the temperature range where the pure-lithium-metal ionization current was still high. These results were explained on the hypothesis of complete dissociation of the lithium chloride molecules on rhenium in the high-temperature region and reduced dissociation of the molecules in the region of threshold temperatures. 
This hypothesis was confirmed by showing that the total current for the combined surface ionization of lithium atoms with lithium. chloride molecules is equal to the sum of the currents obtained from the atomic and molecular beams individually. If lithium chloride molecules dissociated on rhenium at low temperatures, one would expect a significant difference in the total ion current observed for the combined ionization of lithium plus lithium chloride, in comparison with the ion current from an. atomic beam in the threshold temperature region of atomic lithium. Hence, the surface-ionization threshold for lithium chloride is attributed to a change in the ratio between the evaporation rate of molecules from the surface and the rates of dissociation and recombination on the surface and is not caused by a change in the work function of the rhenium surface.

Furthermore, the additivity of the ion fiuxes of lithium and lithium chloride shows that adsorption of lithium chloride on the surface does not alter its ionization properties. Therefore, the degree of surface ionization $\alpha$ does not depend on the incident-beam intensity, a result used in Eq. 3.51. In addition, Potekhina, ${ }^{98}$ using the data of $\mathrm{Z}$ andberg and. Tontegode, ${ }^{8}$ was able to obtain an acceptable value for $D_{M X}(g)$ from a plot of $\ell n x_{1} Z$ versus $1 / k T$, justifying neglect of formation of $\mathrm{X}_{2}$ molecules.

Although an adequate description of the surface ionization characteristics of lithium chloride on hot rhenium surfaces has been provided, ${ }^{98}$ several experiments on other alkali halide-metal systems suggest that the above hypothesis cannot be invoked for all systems. Scheer and Fine ${ }^{47,48}$ using pulsed beams of atomic cesium, molecular cesium chloride, and cesium iodide, determined the mean residence time and the desorption energy of cesium ions from hot polycrystalline surfaces or rhenium and tungsten.

Ion-desorption lifetimes were obtained from oscillograms of the positive-ion pulses produced by a chopper. The time constants for the positive-ion current decay were determined during that part of the modulation cycle when the beam was interrupted. The variation of the time constants with temperature $\left[\tau_{i}=\tau_{i}^{0} \exp \left(E_{i} / k T\right)\right]$ permits a determination of the ion-desorption energy, $E_{i}$. Low surface coverage of the residual gases on the metal filaments was ensured by using the flash-filament technique.

For a given beam intensity and the appropriate range of temperature, thermodynamic considerations show that complete dissociation of cesium iodide should occur on the hot tungsten surface. Partial dissociation of cesium chloride was expected for the same range of temperatures and identical beam intensities. This should result in a reduced ionization efficiency and more complex desorption kinetics of cesium ions produced from cesium chloride beams. This expectation was not experimentally fulfilled. No difference in the positive ionization efficiencies of any of the three beam materials was detected. The kinetics of desorption were always first order. 
Increasing the work function of tungsten to $5 \mathrm{eV}$ in the thermodynamic estimates'cannot ensure complete dissociation of cesium chloride. The suggestion was made ${ }^{47}$ that the thermal equilibration assumed to exist on the surface was influenced by a competing reaction. If a sufficiently rapid combination of adsorbed chlorine with surface-tungsten atoms occurred over the temperature range used, the resulting shift in the cesium chloride dissociative equilibrium could account for the anomalously high ionization efficiency observed.

The mean residence times obtained using the cesium beam were slightly larger than those measured with the cesium iodide beam. However, the values for $E_{i}$ and $\tau_{i}^{0}$ agreed within the estimated probable error. For cesium chloride incident on tungsten, $\tau_{i}^{0}$ was considerably less and the desorption energy about $0.2 \mathrm{eV}$ higher than the values obtained for cesium-ion desorption using cesium or cesium iodide beams.

When beams of the same material were intercepted by a rhenium surface, cesium iodide behaved anomalously. The preexponential term, $\tau_{i}^{0}$, increased markedly, and the desorption energy of cesium ions for the cesium iodide beam was less than that obtained for cesium and cesium chloride beams.

With the exception of cesium iodide on rhenium, the desorption energy always decreased by about $0.5 \mathrm{eV}$, and $\tau_{i}^{0}$ increased about $a$. factor of 100 when either the tungsten or rhenium surfaces were allowed to become contaminated with the background gases. The thickness and insulating properties of the contaminant layer can qualitatively account for the decrease in the desorption energy. The large increase in $\tau_{i}^{0}$ implies a very large change in the shape as well as in the position of the minimum in the potential-energy curve describing the ion-surface interaction. The cesium iodide-rhenium data showed essentially no effect of surface exposure to the residual vacuum gases.

Hughes and Levinstein ${ }^{50}$ observed no significant differences' in the values of the mean residence times for positive rubidium ions on tungsten for beam particles of rubidium metal, of rubidium chloride, and of. rubidium iodide. They likewise used a pulsed-beam technique.

Kaminsky ${ }^{25}$ combined modulated atomic and/or molecular. beams with phase-sensitive mass-spectrometric detection. This provided an opportunity to adequately check the composition and purity of the incident beam. In addition, the temperature dependence of the surface-ionization degree, $\alpha$, can be measured and a value obtained for the effective work function.

When the incident beam on tungsten consisted predominantly of atomic sodium, the variation in the sodium-ion mean adsorption lifetime with temperature obeyed the expression 


$$
\tau_{i}=\left[(0.85 \pm 0.05) 10^{-13} \mathrm{sec}\right] \exp \frac{2.69 \pm 0.03 \mathrm{eV}}{\mathrm{kT}}
$$

for $1200^{\circ} \mathrm{K} \leq \mathrm{T} \leq 1400^{\circ} \mathrm{K}$.

For sodium chloride incident on tungsten, the mean adsorp... tion lifetime is given by

$$
\tau_{i}=\left[(0.20 \pm 0.05) 10^{-12} \mathrm{sec}\right] \exp \frac{2.55 \pm 0.03 \mathrm{eV}}{\mathrm{kT}}
$$

for $1180^{\circ} \mathrm{K} \leq \mathrm{T} \leq 140.0^{\circ} \mathrm{K}$.

When sodium chloride is the beam material, the value of $\tau_{i}^{0}$ increases and the value of the desorption energy decreases. Using incident beams of neutral cesium and cesium chloride, Scheer and Fine ${ }^{47}$ observed just the opposite effect for cesium-ion desorption. This is not unexpected, since the degree of ionization, $\alpha$, varies with temperature in opposite direction for cesium and sodium systems on tungsten. For tungsten surfaces contaminated with background gases, a marked decrease in the value of the mean residence times with corresponding much larger values of $\tau_{i}^{0}$ and a remarkably smaller desorption energy was observed.

The enhanced emission of sodium ions for sodium chloride beams was again attributed to competing reactions of chlorine with the tungsten surface. In all the aforementioned experiments, the incident-beam flux was kept low (about $10^{11}$ particles $\mathrm{cm}^{-2} \mathrm{sec}^{-1}$ ), so that only a small chlorine surface coverage $\left(\theta \cong 3 \times 10^{-3}\right.$ for $\mathrm{NaCl}$ particles $)$ is expected. A more drastic effect should therefore be expected for higher intensities of the incident. beam.

E. Thermodynamic Considerations ${ }^{103}$

Consider the equilibrium

$$
\mathrm{MX}(\mathrm{g})=\dot{M}(\mathrm{~g})+\mathrm{X}(\mathrm{g})
$$

where the equilibrium constant, $K$, is given by

$$
K=\frac{\mathrm{p}_{M} \mathrm{p}_{\mathrm{X}}}{\mathrm{P}_{\mathrm{MX}}} .
$$

For a beam of MX equilibrating with a surface at $T^{\circ} \mathrm{K}$, the pressure of the ith constituent in dynes $\mathrm{cm}^{-2}$ is given by

$$
p_{i}=z_{i}\left(2 \pi m_{i} k T\right)^{1 / 2}
$$


where $Z_{i}$ is the flux of the ith constituent after equilibration with the surface. From stoichiometry," $\vdots$. .

$$
Z_{M}=Z_{X} ; Z_{M X}=Z-Z_{M}
$$

where $\mathrm{Z}$ is the beam intensity of $\mathrm{MX}$ impinging on the surface. If $\mathrm{x}$ is the fraction of MX molecules that dissociate, the equilibrium constant can be written

$$
K=\frac{x^{2}}{1-x} Z(2 \pi u k T)^{1 / 2}
$$

where $u$ is the reduced mass of MX.

From thermodynamics,

$$
-\mathrm{RT} \ln \mathrm{K}=\Delta \mathrm{G}_{\mathrm{T}}^{0}=\Delta \mathrm{H}_{\mathrm{T}}^{0}-\mathrm{T} \Delta \mathrm{S}_{\mathrm{T}}^{0}=\mathrm{T} \Delta \mathrm{fef}+\Delta \mathrm{H}_{298}^{0}
$$

where

$$
\Delta f e f=\frac{\left(G_{T}^{0}-H_{298}^{0}\right)_{P}}{T}-\frac{\left(G_{T}^{0}-H_{298}^{0}\right)_{R}}{T}
$$

is the difference of the free-energy function of products and reactants. For values pertaining to $0^{\circ} \mathrm{K}$, one can use

$$
\frac{\mathrm{G}_{\mathrm{T}}^{0}-\mathrm{H}_{2,98}^{0}}{\mathrm{~T}}=\frac{\mathrm{G}_{\mathrm{T}}^{0}-\mathrm{H}_{0}^{0}}{\mathrm{~T}}-\frac{\mathrm{H}_{2,98}^{0}-\mathrm{H}_{0}^{0}}{\mathrm{~T}} .
$$

Another useful expression is

$$
\frac{G_{T}^{0}-H_{298}^{0}}{T}=\frac{H_{T}^{0}-H_{298}^{0}}{i}-\left(S_{T}^{0}-S_{298}^{0}\right)-S_{2,98}^{0} \text {. }
$$

The excellent summaries of heat content and entropy data that have been published ${ }^{104-106}$ are organized for easy calculation of the $298^{\circ} \mathrm{K}$ freeenergy function by Eq. 3.70 .

Other comprehensive tabulations of thermodynamic properties over wide ranges of temperature are available for the elements, ${ }^{107,108}$ : for metals, ${ }^{109}$ for ions, ${ }^{110}$ and for compounds. ${ }^{100,111}$ Current papers containing thermodynamic data are summarized yearly. ${ }^{112}$ The JANAF Tables ${ }^{100}$ present information on molecular parameters in addition to the thermodynamic functions of selected elements and compounds.

From the thermodynamic data, the equilibrium constant may be calculated from which the degree of dissociation may be estimated as a function of temperature and incident-beam intensity. 
Assuming a uniform work function, three independent quasi-equilibria are in effect for the systems under consideration. The first is

$$
M X(g)=M(g)+X(g)
$$

with

$$
\mathrm{K}_{1}=\frac{\mathrm{P}_{\mathrm{M}^{\mathrm{P}}}}{\mathrm{p}_{\mathrm{MX}}}=\frac{\mathrm{Z}_{\mathrm{M}^{\mathrm{Z}}} \mathrm{Z}_{\mathrm{X}}}{\mathrm{Z}_{\mathrm{MX}}}(2 \pi u k \mathrm{~T})^{1 / 2}
$$

A fraction $\beta_{M}$ of $M$ is ionized on the surface. The associated equilibrium is

$$
M(g)=M^{+}(g)+e^{-(g)},
$$

with

$$
K_{2}=\frac{p_{M^{+}} e}{p_{M}}=\frac{Z_{M^{+}} Z_{e}}{Z_{M}}\left(2 \pi m e^{k T}\right)^{1 / 2},
$$

where $\mathrm{Z}_{\mathrm{e}}$ denotes the flux of electrons due to thermionic emission. A third independent equilibrium is associated with thermionic emission of electrons and is represented by

$$
e^{-}(\text {F.S. })=e^{-}(g) \text {, }
$$

with

$$
K_{3}=\frac{p_{e}}{p_{e}(\text { F.S. })}=\frac{Z_{e}\left(2 \pi m_{e} k T\right)^{1 / 2}}{p_{e}(\text { F.S. })}
$$

where $e^{-}$(F.S.) denotes the electrons in the Fermi Sea of the metal.

The fluxes are related by the following constraints:

$$
\begin{aligned}
& Z_{M^{+}}+Z_{M^{+}}=Z_{X}=Z-Z_{M X^{\prime}} \\
& \frac{Z_{M^{+}}}{Z_{X}}=\frac{Z_{M^{+}}}{Z_{M^{+}}+Z_{M^{+}}}=\beta_{M^{\prime}}
\end{aligned}
$$

and

$$
Z_{e}=I_{e} \frac{N_{0}}{F}=\frac{I_{e}}{e},
$$


where $\mathrm{F}$ is the Faraday equivalent, $\mathrm{N}_{0}$ is Avogadro's number, and $\mathrm{e}$ is the electron charge in coulombs. The electron current, $I_{e}$, is given by the familiar Richardson-Dushman equation for thermionic emission

$$
I_{e}=A T^{2} \exp \left(-\frac{\phi}{k T}\right)
$$

where

$$
\mathrm{A}=\frac{4 \pi \mathrm{m}_{\mathrm{e}} \mathrm{ek}^{2}}{\mathrm{~h}^{3}}=120 \mathrm{~A} \mathrm{~cm}^{-2}{ }^{\circ} \mathrm{K}^{-2}
$$

The value of A varies experimentally with conditions and material used.

Thermionic emission of electrons is treated as an independent process occurring simultaneously with dissociation of the incident beam and subsequent surface ionization of the metal atom on the uniform workfunction surface. It is evident, nevertheless, that the partial pressure of the electron gas influences the other partial pressures.

In terms of the incident-beam intensity, $Z$, where $f$ is the fraction of MX dissociated,

$$
\begin{aligned}
& Z_{M X}=Z-Z_{X}=Z-Z f=Z(1-f), \\
& Z_{X}=Z f \\
& Z_{M^{+}}=Z_{X_{M}^{\beta}}=Z_{f} \beta_{M^{\circ}}
\end{aligned}
$$

and

$$
Z_{M}=Z_{X}-Z_{M^{+}}=Z_{f}\left(1-\beta_{M}\right)
$$

$$
\}
$$

Equations $3.63^{\prime}$ and 3.72 then give

$$
K_{1}=\frac{Z f^{2}(1-\beta M)}{1-f}(2 \pi u k T)^{1 / 2}
$$

and

$$
K_{2}=\frac{\beta_{M}}{1-\beta_{M}}\left(2 \pi m_{\left.e^{k T}\right)^{1 / 2}}=\alpha_{L} Z_{e}\left(2 \pi m e^{k T}\right)^{1 / 2}\right.
$$

For incident beams on uniform work-function surfaces, Eq. 3.81 is sufficient for calculating the degree of dissociation, $f$. The thermodynamic data used 
in calculating the equilibrium constants were taken from the JANAF Tables. ${ }^{100}$ Table $V$ gives values of the fraction of lithium fluoride dissociation under a variety of conditions for uniform work-function surfaces.

TABLE V. Variation in Fraction $f$ of LiF Dissociated as a Function of Temperature for Different Work Functions, $\phi$, and Incident-beam Intensities, $\mathrm{Z}$

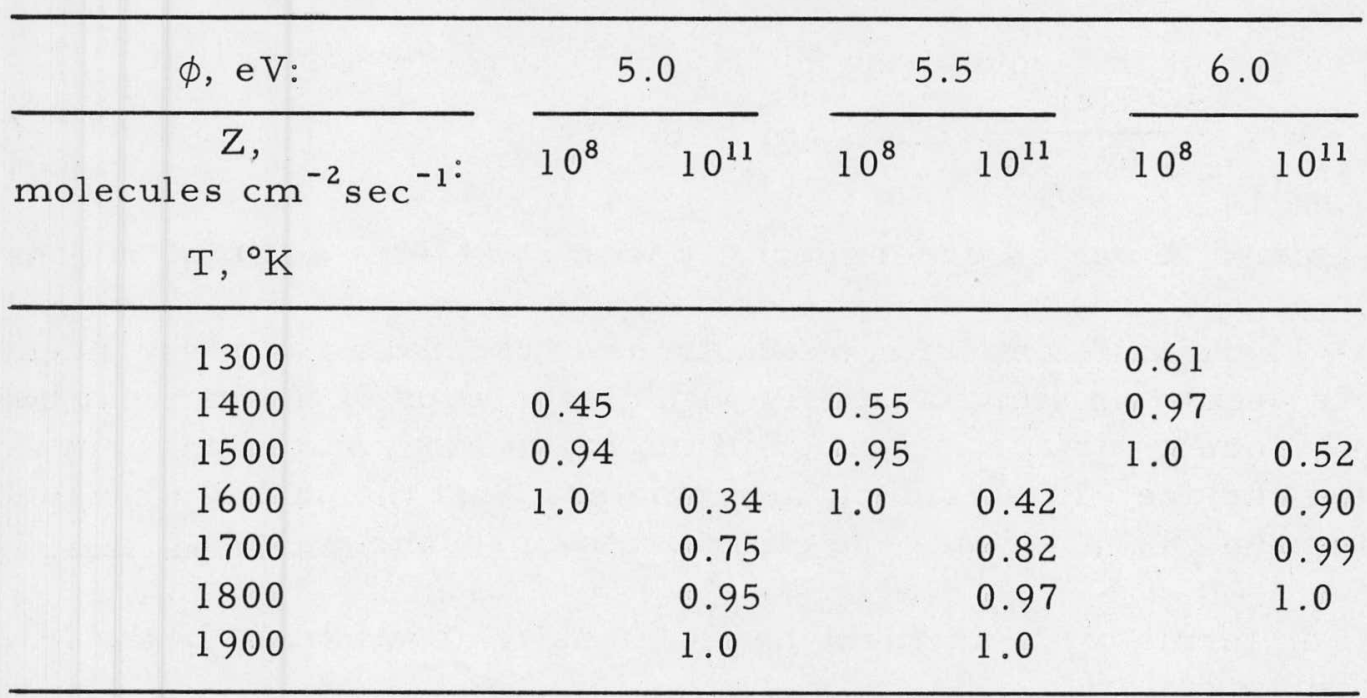

For a beam of $\operatorname{LiF}\left(Z=10^{11}\right)$ equilibrating with a surface of uniform work function $(\phi \leq 5.5 \mathrm{eV})$, essentially complete dissociation occurs above $1800^{\circ} \mathrm{K}$. Increasing the uniform work function to $6.0 \mathrm{eV}$ gives complete dissociation at $1700^{\circ} \mathrm{K}$. Since $\mathrm{LiF}$ has the largest dissociation energy of the lithium halides, the other lithium halides must be completely dissociated at lower surface temperatures for the same beam intensities. For lower beam intensities, complete dissociation occurs at lower temperatures.

For polycrystalline surfaces, electron emission arises predominantly from the crystal faces of low work function, whereas ion emission occurs predominantly from those crystal faces of high work function. For thermodynamic equilibrium to prevail, the ratio $Z_{M+} / Z_{M}=\alpha_{L}$ in Eq. 3.82 must decrease due to the enhanced electron emission from the low-work-function patches. This leads to a decrease in the fraction $\mathrm{f}$ of $\mathrm{MX}$ dissociated. If, however, the emission of electrons is suppressed by the application of a suitable potential to the emitting polycrystalline filament, and this is assumed to have a negligible effect on the surface ionization properties, Eq. 3.81 may still be used to estimate the fraction of MX dissociated. The applicable work function in this instance would be the effective work function for surface ionization measured experimentally.

In the temperature region of incomplete dissociation of MX molecules, Eq. 3.80 may be rearranged to give a value of $\beta_{M X}$ in terms of $f$, the fraction dissociated. Thus 


$$
\mathrm{Z}_{\mathrm{M}^{+}}+\mathrm{z}_{\mathrm{M}}=\mathrm{Zf}
$$

or

$$
\frac{\mathrm{Z}_{\mathrm{M}^{+}}}{\mathrm{Z}}=\frac{\alpha}{1+\alpha} \mathrm{f}=\beta_{\mathrm{M}^{\mathrm{f}}}=\beta_{\mathrm{MX}}
$$

Comparison of this equation with Eqs. 3.54 and 3.55 shows that

$$
f=\left(1+\frac{P_{M X}}{k_{1}}\right)^{-1}
$$

or

$$
f=\sqrt{\frac{1+\alpha}{x_{1} Z}}
$$

depending on the relative values of $\mathrm{P}_{\mathrm{MX}}, \mathrm{k}_{1}$, and $\mathrm{x}_{1}$, and provides a check for the validity of the assumptions used.

F. Influence of Polycrystalline Surfaces

The Saha-Langmuir surface-ionization equation is valid only for surfaces with a uniform work function, such as single crystal surfaces. Polycrystalline surfaces, by contrast, consist of patches of single crystal faces with different local work functions, ${ }^{27,28,80,85,113-116}$ which give rise to different emission intensities.

The principal contribution to the thermionic-emission current is made by faces with a low work function; here the effective thermionic work function of a polycrystalline surface, $\phi_{\mathrm{e}^{*}}^{*}$ approaches the minimum local values of the surface work function, $\phi_{\text {min. }}$. The principal contribution to the ion current through positive surface ionization of atoms is made by faces with a high work function. In this case, the effective surface-ionization work function of a polycrystalline surface $\phi_{I}^{*}$ approaches the maximum local values of the surface work function, $\phi_{\max }$. The difference, $\phi_{I}^{*}-\phi_{\mathrm{e}}^{*}$, serves as a measure of the degree of contrast of the emitter surface with respect to thermionic emission and surface ionization.

The emission from the total polycrystalline surface may be described by the introduction of an effective work function, $\phi_{I}^{*}$, and the effective degree of ionization, $\alpha^{*}$, represented by

$$
\alpha^{*}=\left(\frac{Z_{+}}{Z_{0}}\right)_{\text {total surface }}=\frac{g_{+}}{g_{0}} \exp \frac{\phi_{I}^{*}-I}{k T} .
$$


Weiershausen ${ }^{75}$ considered two possibilities in the calculation of $\alpha^{*}$ and $\phi_{I}^{*}$ from the local work functions, $\phi_{\mathrm{n}}$, of the various patches and their relative fractions, $f_{n}$ of the total surface.

Individual patches emit independently of each other according to the local work functions, $\phi_{\mathrm{n}}$. If $\alpha_{\mathrm{n}}<<1$, then the following simplified equation is obtained: 85

$$
\alpha *=\frac{g_{+}}{g_{0}} e^{-I / k T} \cdot \sum_{n} f_{n} e^{\phi_{n} / k T} .
$$

If Eq. 3.87 is compared with Eq. 3.86, the effective work function is given by

$$
\phi_{I}^{*}=k T \ln \sum_{n} f_{n} e^{\phi_{n} / k T}
$$

which is greater than the work function averaged arithmetically over the total surface,

$$
\bar{\phi}=\sum_{\mathrm{n}} \mathrm{f}_{\mathrm{n}} \phi_{\mathrm{n}}
$$

Individual exposed crystallographic planes no longer emit independently of each other, because of the electric fields existing between these patches of different work functions. These patch fields equalize the difference in work functions. At a distance $x$ from the surface, which is of the order of magnitude of the mean patch diameter, the potential over all patches is equal to the potential that would exist at the same distance over a homogeneous metal surface with the mean work function $\bar{\phi}$. The patch field retards the ions emitted by patches with a higher work function, $\phi_{h}$ in comparison with those emitted by patches with a mean work function $\bar{\phi}$.

Ions emitted from patches with a work function $\phi_{I}<\bar{\phi}$ are accelerated, although this has no effect on the desorption probability of the ion, since these ions have first to overcome a potential barrier, $\triangle_{\ell}$, higher than the potential at the distance $x$. Since $\bar{\phi}-\Delta_{l}=\phi_{l}$, the local work function $\phi_{l}$ is used. The effective work function then is given by

$$
\phi_{I}^{*}=k T \ln \left[e^{\bar{\phi} / k T} \cdot \sum_{h} f_{h}+\sum_{\ell} f_{\ell} e^{\phi_{\ell} / k T}\right]
$$

where $\sum_{h}$ is extended over all patches with work functions greater than $\bar{\phi}_{\text {, }}$ and the summation $\sum_{l}$ is extended over all patches with a work function lower $\operatorname{than} \bar{\phi}$. 
In contrast to the previous case, the temperature relation of the effective work function is negligibly small, amounting to less than $0.1 \mathrm{eV}$ between 1000 and $2500^{\circ} \mathrm{K}$. There is also the effect of surface diffusion, ${ }^{117}$ which could condition a further approach of $\Phi_{I}^{*}$ to $\bar{\phi}$, since the patch field favors the transport of ions to patches with a local work function greater than $\bar{\phi}$. From these patches, however, an emission according to $\bar{\phi}$ will take place.

G. Flash Desorption

For completeness, a mathematical analysis of flash desorption follows. ${ }^{58,118,119}$ The rate of change of the number of molecules $\mathrm{dn} / \mathrm{dt}$ in the system of volume $\mathrm{V}$ is given by

$$
\frac{\mathrm{dn}}{\mathrm{dt}}=\frac{\mathrm{Vdp}}{\mathrm{kTdt}}=\mathrm{L}_{\mathrm{w}} \frac{\mathrm{A}_{0}\left(\mathrm{p}-\mathrm{p}^{\prime}\right)+\mathrm{A}_{\mathrm{w}} \mathrm{p}}{(2 \pi \mathrm{mkT})^{1 / 2}},
$$

where $p$ is the system pressure, $L_{w}$ the desorption rate from the system walls, $A_{w}$ the effective adsorption area of the walls, $\mathrm{p}^{\prime}$ the pressure at the throat of the pump. $A_{0}$ the effective area of the pumping orifice, $m$ the molecular mass, $\mathrm{k}$ the Boltzmann constant, and $\mathrm{T}$ the system temperature. For simplicity, the effect of ion-gauge pumping may be included in the term $\mathrm{A}_{\mathrm{w}}$. In a mass-spectrometer vacuum system with the principal residual gases of $\mathrm{H}_{2} \mathrm{O}, \mathrm{N}_{2}, \mathrm{CO}, \mathrm{O}_{2}$, and $\mathrm{CO}_{2}$ present, the rate of change of the total number of molecules is given by

$$
\frac{d n_{T}}{d t}=\sum_{i} \frac{d n_{i}}{d t}=\sum_{i} \frac{V d p_{i}}{k T d t}=\sum_{i} L_{w_{i}}-\sum \frac{A_{0 i}\left(p_{i}-p_{i}^{l}\right)+A_{w i} p_{i}}{\left(2 \pi m_{i} k T\right)^{1 / 2}} .
$$

At the steady-state or equilibrium-base pressure $\mathrm{p}=\mathrm{p}_{\mathrm{eq}} \mathrm{q}^{\circ} \mathrm{dn} / \mathrm{dt}=0$, $\mathrm{dp} / \mathrm{dt}=0$, and Eq. 3.91 may be rewritten in the form

$$
\frac{\mathrm{dn}}{\mathrm{dt}}=\frac{\mathrm{Vdp}}{\mathrm{kTdt}}=\frac{\left(\mathrm{A}_{0}+\mathrm{A}_{\mathrm{w}}\right)\left(\mathrm{p}_{\mathrm{eq}}-\mathrm{p}\right)}{(2 \pi \mathrm{mkT})^{1 / 2}}=0
$$

or

$$
\frac{d p}{d t}=\frac{p-p_{e q}}{\frac{V}{A_{0}+A_{w}}\left(\frac{2 \pi m}{k t}\right)^{1 / 2}}=\frac{p-p_{e q}}{\tau}
$$

where

$$
\tau=\frac{V}{A_{0}+A_{W}}\left(\frac{2 \pi m}{k T}\right)^{1 / 2}=\frac{V}{S}
$$


is the characteristic pumping time of the system, and $S$ is the pumping speed in volume units per second. If a gas is allowed to flow into the system at a constant rate, a new steady-state pressure is attained. When the flow is stopped, the system pressure decreases to the base-equilibrium pressure, and the characteristic pumping time of the system at a pressure $p$ for the particular gas in question may be obtained. This follows directly by integration of Eq. 3.93 to give

$$
\ln \left(p-p_{e q}\right)=-\frac{t}{\tau}+C
$$

and $\tau$ is the slope of the curve of $\ln \left(p-p_{e q}\right)$ versus $t$. It is assumed that $\tau$ is independent of pressure; i.e., $A_{w}$, the effective adsorption area of the walls, is independent of pressure for the linear relationship, Eq. 3.95, to hold.

We now introduce a metal filament into the system and allow adsorption and desorption to occur as the filament temperature is raisedor lowered. Equation 3.93 then becomes

$$
-\frac{\mathrm{dp}}{\mathrm{dt}}=\frac{\mathrm{p}-\mathrm{peq}}{\tau}-\frac{\mathrm{kT}}{\mathrm{V}}\left[\mathrm{L}_{\mathrm{m}}-\frac{\mathrm{sAp}}{(2 \pi \mathrm{mkT})^{1 / 2}}\right]
$$

where $\mathrm{L}_{m}$ is the desorption rate from the metal filament, $A$ is the area of the filament surface, and $s$ is the sticking coefficient for the gas-metal system.

The desorption process is considered first. If the metal surface is heated to a high temperature for a period long with respect to the

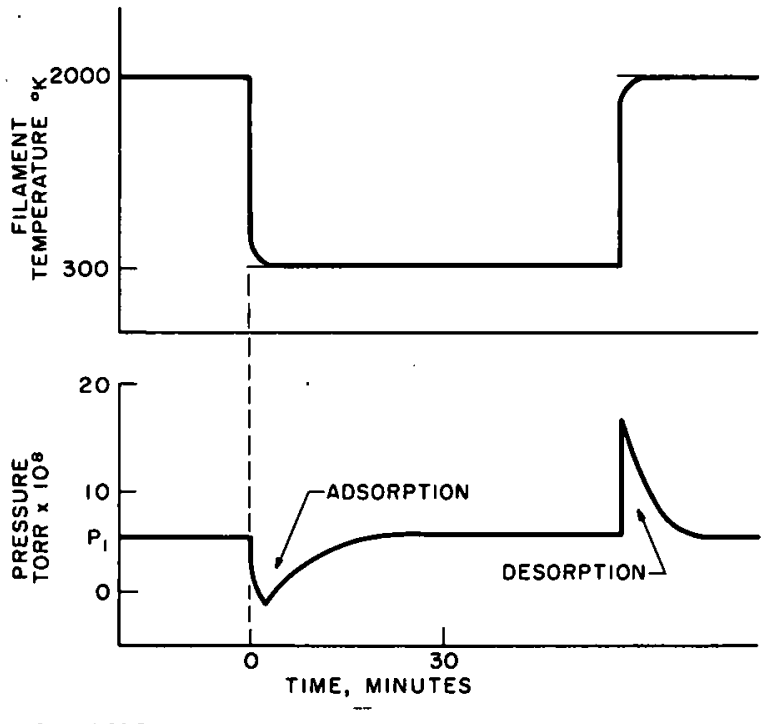

$121-5503$

Fig. 4. Schematic Diagram of the Temporal Behavior of a Filament Surface Temperature and $G$ as Pressure desorption time, the steady state is attained where $\mathrm{p}=\mathrm{p}_{\text {eq }}$; hence, $\mathrm{dp} / \mathrm{dt}=0$, and

$L_{m}=\frac{s A p}{(2 \pi m k T)^{1 / 2}}=0$

since there are no molecules left on the filament surface to desorb. (See Fig. 4.) During the latter part of the falling-off portion of the desorption curve, $\mathrm{L}_{\mathrm{m}}$ and $\mathrm{s}$ are. small and Eq. 3.96 may be approximated by Eq. 3.93. Again, a characteristic pumping time may be estimated to adjudge the effect, if . any, of gas desorption from the walls of the system on filament heating. 
The instantaneous rate of desorption per unit area, $L_{m} / A=d \sigma / d t$, is, from Eq. 3.96,

$$
\frac{A k T}{V} \frac{d \sigma}{d t}=\frac{d p}{d t}+\frac{p-P_{e q}}{\tau}+\frac{A k T s p}{V(2 \pi m k T)^{1 / 2}}
$$

During the early portion of the heating cycle, the readsorption of chemisorbed gases depends on the population of the various adsorbed phases, the pumping speed, and the duration of the desorption cycle. Readsorption is trivial unless the pumping speed is very slow and the desorption cycle long.

The separate chemisorbed phases are usually observed to fill up in decreasing order of their energies and desorbed in increasing order of their energies. As one particular phase is desorbing, the higher-energy phases, which have not yet desorbed, are almost fully populated, and thus readsorption into these phases is unlikely. For short desorption cycles, Eq. 3.98 becomes

$$
\frac{\operatorname{AkT}}{\mathrm{V}} \frac{\mathrm{d} \sigma}{\mathrm{dt}}=\frac{\mathrm{dp}}{\mathrm{dt}}+\frac{\mathrm{p}-\mathrm{p}_{\mathrm{eq}}}{\tau}
$$

Two conditions may be realized with respect to the pumping speed. For small pumping speeds $(\tau \rightarrow \infty)$, the desorption rate is proportional to the first derivative of the pressure with time. This condition can be approximated in practice when the duration of desorption sweep is made short compared with the characteristic pumping time $\tau$. With chemically active gases, this condition can be approached with very fast sweep rates $\left(>10^{3} \mathrm{o} / \mathrm{sec}\right)$.

Alternatively, for very high pumping speeds $(\tau \rightarrow 0)$,

$$
\frac{\mathrm{p}-\mathrm{P}_{\text {eq }}}{\tau} \rightarrow \frac{\mathrm{AkT}}{\mathrm{V}} \frac{\mathrm{d} \sigma}{\mathrm{dt}}
$$

thus the desorption rate is proportional to pressure.

The clean metal surface is now cooled to ambient temperature. The desorption rate becomes vanishingly small, and, from Eq. 3.98, the rate of change of the adsorbed species surface concentration do/dt is given by

$$
A \frac{d \sigma}{d t}=\frac{s A p}{(2 \pi m k T)^{1 / 2}}=\frac{V}{k t}\left[\frac{p_{e q}-p}{\tau}-\frac{d p}{d t}\right] .
$$

The expression for calculating the sticking coefficient from an experimental $p-t$ curve at any time $t$ during adsorption is therefore 


$$
s=\frac{V}{A}\left(\frac{2 \pi m}{k T}\right)^{1 / 2}\left[\frac{p_{e q}-p}{p \tau}-\frac{1}{p} \frac{d p}{d t}\right] .
$$

Equation 3.102 may be rewritten in the form.

$$
-\frac{d p}{d t}=\frac{p-p_{e q}}{\tau s}+\frac{s p}{\tau_{f}}
$$

to show that the rate of change of pressure is inversely proportional to the characteristic pumping time of the system, $\tau_{s}$, and of the filament, $\tau_{f}$.

Integration of Eq. 3.101 gives the surface concentration, $\sigma_{t}$, after a cooling. interval thas elapsed,

$$
\sigma_{t}=\frac{v}{A k T}\left[\frac{1}{\tau}\left(p_{e q} t-\int_{0}^{t} p d t\right)+p_{e q}-p\right] .
$$

The metal surface finally becomes saturated, and a low-temperature steady state is reached after a time $t_{0}$, and $p=p_{e q}$ once more. The steady-state surface coverage, $\sigma_{0}$, is then

$$
\sigma_{0}=\frac{V}{A k t}\left[\frac{1}{\tau}\left(p_{e q} t_{0}-\int_{0}^{t_{0}} p d t\right)\right]:
$$

The expression in parentheses in Eqs. 3.104 and 3.105 is the area between the experimental $p-t$ curve and the steady-state pressure peq after times $t$ and $t_{0}$ have elapsed. These areas can be measured directly from the pressure-time records, and Eqs. 3.102,3.104, and 3.105 may be used to calculate $s, \sigma_{t}$, and $\sigma_{0}$. Simplifying assumptions with respect to high and low pumping speeds may be invoked as outlined on page 53 for the desorption.cycle.

An alternate method for determining the surface concentration of adsorbed gas is to desorb the gas by flashing the filament at increasing intervals of adsorption time. The pressure burst is then proportional to the length of the previous cold interval, the arrival rate of molecules at the filament surface, and the integrated probability that an incident molecule will remain on the surface (the sticking probability). Provided that all sources and pumps in the system (other than the adsorbing filament) are constant in speed, the area under the desorption curve is equal to the area of the adsorption interval.

If the steady-state surface coverage, $\sigma_{0}$, is that for a monolayer, then the surface concentration $\sigma_{t}$ at a time $t$ is given by 


$$
\sigma_{t}=\theta \sigma_{0},
$$

where $\theta$ is the fraction of a monolayer covered. The relationship between surface coverage and the sticking coefficient is then obtained from Eq. 3.101, provided evaporation from the filament can be ignored. The result is

$$
\mathrm{A} \sigma_{0} \frac{\mathrm{d} \theta}{\mathrm{dt}}=\frac{\mathrm{sAp}}{(2 \pi \mathrm{mkT})^{1 / 2}}=\frac{\mathrm{V}}{\mathrm{kt}}\left(\frac{\mathrm{p}_{\mathrm{eq}}-\mathrm{p}}{\tau}-\frac{\mathrm{dp}}{\mathrm{dt}}\right) .
$$

Hagstrum ${ }^{30}$ used the term "monolayer" to denote the surface coverage at which the adsorption rate is observed to decrease from a relatively high value to a relatively low value. The time required to reach this condition from the atomically clean condition is termed the "monolayer adsorption time, $" t_{0}$.

The kinetic parameters of the desorption process may be obtained by representing the rate of evolution from a surface of area $A$ by an Arrhenius-type equation. Thus the rate of desorption from unit surface area may be written as

$$
-\frac{d \sigma}{d t}=\sigma^{x} \nu_{x} \exp \left(-\frac{\Delta H}{R T}\right)
$$

where $\mathbf{x}$. is the order of the desorption reaction, $\sigma$ is the surface concentration in molecules per square centimeter, $\nu_{x}$ is the preexponential factor, which may include an appropriate correction for surface roughness and to a first approximation is assumed independent of temperature, and $\Delta \mathrm{H}$ denotes the heat of activation for the desorption process; $\Delta H$ may or may not be independent of $\sigma$ and $T$. Equation 3.96 now becomes

$$
\sigma^{x_{\nu}} \nu_{x} \exp \left(-\frac{\Delta H}{R T}\right)-\frac{s p}{(2 \pi m k T)^{1 / 2}}=\frac{V}{A k T}\left(\frac{p-p_{e q}}{\tau}+\frac{d p}{d t}\right) .
$$

Again, during desorption, readsorption may be negligible and the stickingcoefficient term can be neglected. The kinetic parameters $\Delta H, \nu$ and $\mathrm{x}$ can then, in principle, be obtained from the pressure-time relationship. The arguments concerning duration of desorption cycle and pumping times are likewise pertinent.

If the activation energy for desorption is a function of the surface concentration, this removes the independence of concentration that holds for a simple first-order desorption. However, only the first-order evaporation terminates at the same time, regardless of initial conditions, and a distinction between first- and second-order reactions can be accomplished by this criterion. ${ }^{57}$ 
If one has a known variation of filament temperature with time, e.g., $T=T_{0}+\beta t$, the various adsorption and desorption parameters may be derived from the curve of desorption rate as a function of filament temperature.

We now introduce a flow of gas from a source other than the walls of the vacuum system. The appropriate equation now becomes (from $\mathrm{Eq} \cdot 3.96)$

$$
-\frac{\mathrm{dp}}{\mathrm{dt}}=\frac{\mathrm{p}-\mathrm{peq}}{\tau}-\frac{\mathrm{kT}}{\mathrm{V}}\left[\mathrm{L}_{\mathrm{m}}+\mathrm{L}_{\mathrm{o}}-\frac{\mathrm{sAp}}{(2 \pi \mathrm{mkT})^{1 / 2}}\right]
$$

where $L_{0}$ is the flow from a source in molecules per second. At some high temperature and at a time sufficiently long for complete desorption, a steady state is reached, $p_{1}>p_{e q}$, due to the increased pressure caused by the new source. Under these conditions,

$$
\frac{\mathrm{kT}}{\mathrm{V}} \mathrm{L}_{0}=\frac{\mathrm{p}_{1}-\mathrm{p}_{\mathrm{eq}}}{\tau}
$$

At any instant during the desorption cycle,

$$
-\frac{\mathrm{dp}}{\mathrm{dt}}=\frac{\mathrm{p}-\mathrm{p}_{\mathrm{l}}}{\tau}-\frac{\mathrm{kT}}{\mathrm{V}}\left[\mathrm{L}_{\mathrm{m}}-\frac{\mathrm{sAp}}{(2 \pi \mathrm{mkT})^{1 / 2}}\right]
$$

which is identical with Eq. 3.96 except for $p_{e q}$ being replaced by $p_{1}$ ( $>p_{e q}$ ) due to the added flow. All the above considerations apply in the present situation and will not be dealt with further.

H. Récent Developments

There are several illuminating theoretical studies of atom adsorption on dissimilar metal substrates. ${ }^{42,63,120,121}$ In these treatments, different bonding mechanisms for alkali adsorption on metals are proposed; yet all are capable of explaining the experimental data relevant to cesium adsorption on tungsten. ${ }^{17}$ These data consist of heats of adsorption and thermionic emission from cesiated surfaces of tungsten.

Since cesium is a large atom with an ionization potential less than that of the work function of tungsten, it is adsorbed on the metal surface, principally as an ion. The ion-metal interaction is described by the classical image force. The emission data correlate with a picture of a metal having an adsorbed dipole layer that lowers the electron work function of the surface. An extension of this view was developed by DeBoer ${ }^{122}$ and more recently by others. ${ }^{42,123,124}$ This picture also has been used to interpret potassium on tungsten data. ${ }^{64}$ 
Gyftopoulos and Levine ${ }^{120}$ used a chemical approach. They proposed that cesium is chemisorbed on the surface forming a partially ionicpartially covalent bond with four substrate atoms. They identified particular conduction-band electrons with particular ion cores. This cannot be totally correct, since the bond is actually formed between the atomic electrons of cesium and many conduction electrons of the metal.

A third approach resulted from an observation by Gurney. ${ }^{43}$ The interaction of an atom with a metal causes the valence level of the atom to broaden. This broadening results as a consequence of the finite lifetime of the atom during the metal-atom interaction and, thus, an uncertainty in the energy level according to the uncertainty principle. This could result in the formation of polar bonds between adsorbate and substrate, which need not necessarily be ionic in character. These ideas were further developed by Dobretzov. ${ }^{14}$

Gomer and associates ${ }^{62-65}$ advanced a criterion, based on Gurney's suggestion, for establishing the nature of the adsorbate-substrate bond as ionic, covalent, or polar metallic. If the ns atomic level of the alkali in question is split and broadened to the order of several electron volts on interacting with the substrate, overlapping occurs with the conduction band of the substrate leading to polar-metallic bonds. The authors suggested that potassium on tungsten must be essentially polar metallic. They found a large decrease in the adsorbate charge and the heat of adsorption with increasing potassium coverage of the polycrystalline and single crystal faces of tungsten. This was attributed to increased filling of the very broadened and split adsorbate level as the level is lowered by the decrease in work function.

Gadzuk ${ }^{121,125}$ showed that the interaction of the ns electron of the alkali atom with a metal is equivalent to a rearrangement collision. Using standard techniques for dealing with rearrangement processes and timedependent perturbation theory, he calculated the lifetime and the natural broadening of the perturbed ns level. For adsorbed alkalies, this perturbed level is less than $1 \mathrm{eV}$ in width, in accord with the level of $0.35 \mathrm{eV}$ suggested by Rasor and Warner ${ }^{42}$ and Gyftopoulos and Levine, ${ }^{120}$ and considerably sharper than advanced by Gomer and Swanson. ${ }^{63}$ This suggests the ionicbond picture for alkalies adsorbed on metals.

These bond types are represented schematically in Fig. 5 for the interacting atom-metal system at $0^{\circ} \mathrm{K} .{ }^{121}$ Figure 5 a depicts a cómpletely ionic bond with no overlap of the conduction band and the broadened atomic level of width $\Gamma$. Such a situation is required for the Rasor and Warner model. If the atom and metal have some overlap, a partially ionic-partially covalent bond is formed, as shown in Fig. 5b. In Fig. 5c, the shifted and broadened adsorbate level falls below the Fermi level, giving rise to the polar-metallic bond proposed by Gomer and Swanson. The resulting dipole 
would appear to be insufficient to account for the lowering of the work function when a partial monolayer is adsorbed on the metal surface.
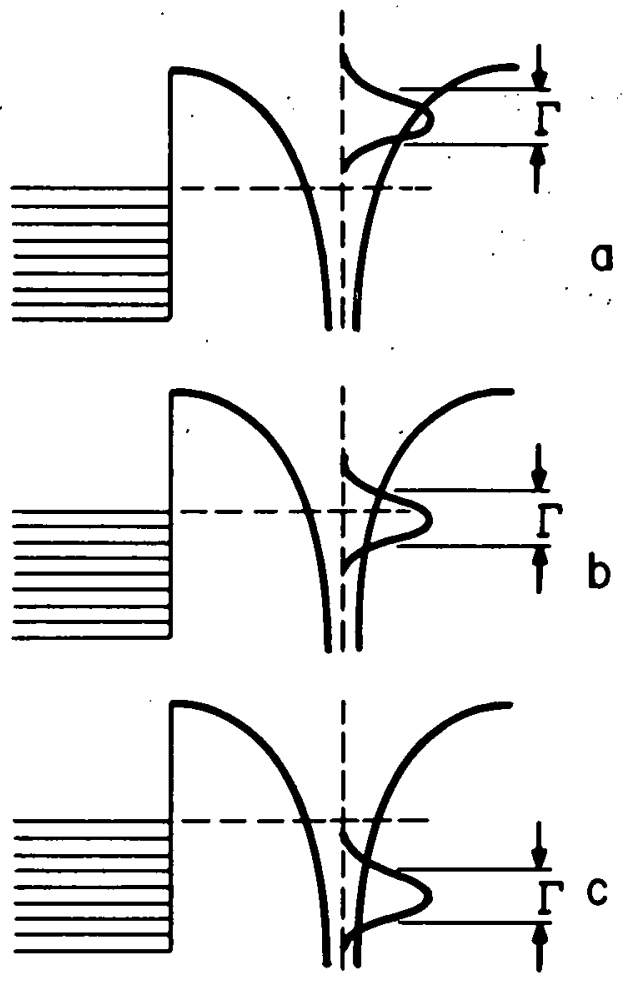

Fig. 5

Bond Types for Atom-Metal System at $0{ }^{\circ} \mathrm{K}$. a. Position of broadened level required for ionic bond, b. Position of broadened level required for partially ionic-partially covalent bond, and c. Position of broadened level required for polar-metallic bond.

As the temperature is raised, some electrons can be found at energies above the Fermi level. Hence, there will be a slight population of the atom-metal levels that have energies within the band of virtual atomic states. This is shown in Fig. 6, where the finite-temperature Fermi distribution determines the occupation probabilities. The population of these states reduces the dipole moment, giving rise to the thermaldepolarization effect mentioned by Rasor and Warner. ${ }^{42}$

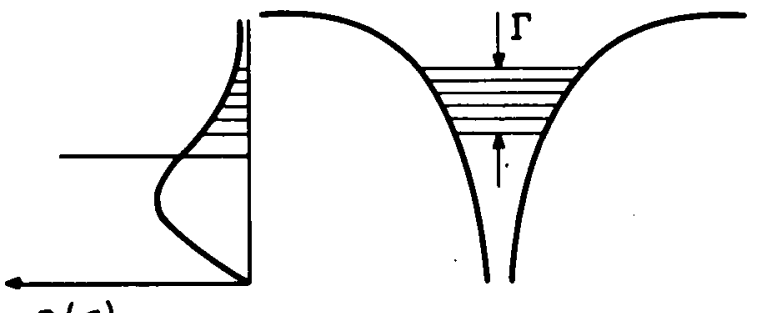

In any case, the picture of atom-metal interaction presented is overly simplified. The substrate structure does play a role apart from explicit electrostatic effects in determining adsorption properties. ${ }^{62,65}$ A more general approach is provided by the theory of surface states developed primarily for semiconductors.

Surface states are those electronic levels of a bounded crystalline solid whose wave functions have finite, nonvanishing values near a surface only. Intrinsic surface states owe their existence entirely to the presence of the surface that interrupts the periodic lattice. Tamm surface states ${ }^{126}$ 
are associated with metallic binding, since they are computed from a planewave approximation with a periodic potential. They can be described as the separation of energy levels above or below a single band. Schockley surface states $^{127}$ arise only when there are two separate volume bands and when the surface introduces a crossing of levels in these bands. This approach is appropriate for covalent binding, in which the lattice is viewed as a collection of weakly coupled atoms and the tight-binding approximation is applied. Levine and Mark proposed a model for ionic crystals. ${ }^{128}$

Extrinsic surface states are associated with the presence of any sort of flaw on the otherwise perfectly periodic surface, such as adsorbed ions or other layers of chemical origin, or structural imperfections. Probably the most complete treatment of a problem approaching a real situation is that due to Koutecky, ${ }^{129,130}$ who studied the chemisorption of hydrogen on the surface of a crystal with the diamond structure. A thorough treatment is beyond the scope of this discussion, particularly since the theory has not reached the stage where calculating forces or energies is feasible. General reviews of the significance and applications of the theory of surface states have been presented. ${ }^{130-137}$ 


\section{CHAP TER IV \\ EXPERIMENTAL STUDY}

The experimental study to be reported is concerned with some of the aspects of surface ionization reviewed in Chapter III. The principal effort is directed toward amplifying the chemical effects of anionic constituents in a.molecular beam on surface-ionization processes.

A solution of a lithium salt was evaporated onto a rhenium ribbon. This ribbon was then positioned about $8 \mathrm{~cm}$ above the ionizing electronbeam path within the ion source of the mass spectrometer. A second clean rhenium ribbon was positioned just below the path of the electron beam. The sample filament was heated electrically to evaporate the salt. The neutral species were collimated onto the second ribbon, and their molecular complexity was determined by electron-impact ionization and mass analysis. With the electron gun off, lithium ions were produced at the electrically heated second filament by surface ionization. The surfaceproduced ions were focused, and their intensity as a function of surface temperature and impingement rate measured mass-spectrometrically. A flash-filament technique was used to ensure an atomically clean surfaceionization filament. Temperatures were measured accurately by optical pyrometry and bridge techniques.

\section{A. Description of Apparatus}

The principal components of the apparatus used in this experimental study consisted of a modified Bendix time-of-flight mass spectrometer, source filaments, a Maxwell bridge, an optical pyrometer, a flash-filament circuit, and the associated recording equipment. These components are described in detail below.

\section{Time-of-Flight Mass Spectrometer}

A Bendix time-of-flight mas s sectrometer ${ }^{138-140}$ (Model.S12-107), capable of resolving a mass of 400 at one atomic-mass-unit separation was used. The spectrometer can theoretically take spectra up to 5000 atomic mass units; however, the resolving power deteriorates to the extent that resolution of masses 600 and 601 is impractical.

The principal advantages of this type of mass spectrometer are as follows:

1. A chemist's tool; does not require a specialist.

2. Continuous display of entire spectrum.

3. Any number of peaks simultaneously and continuously monitored. 


\section{One detector for all ions. \\ 5. Open structure; versatile. \\ 6. Gross optics. \\ 7. Rugged; easy maintenance.}

The principal disadvantages are its limited resolution and its abundance sensitivity. In the original design, ions were produced only during the $0.25 \%$ duty cycle.

The principle of mass separation in a time-of-flight spectrometer is that singly charged ions of different masses $m$ (in atomic mass units), when given the same energy by acceleration through the same potential difference, have velocities inversely proportional to $\sqrt{\mathrm{m}}$. Thus, for two species 1 and 2 , the relative times, $t$, required after acceleration to traverse a fixed field-free distance are related by the equation

$$
t_{1} / t_{2}=\sqrt{\frac{m_{1}}{m_{2}}}
$$

In the ion source of the mass spectrometer, the molecular beam from the sample filament is directed vertically into the ionization region, where it is intersected by a horizontal electron beam or the surface-ionization filament. The ions that result are accelerated electrostatically in a direction normal to the plane of molecular and electron beams. The beam of ionizing electrons can be applied for $0.25 \mu \mathrm{sec}$, at the end of which time the accelerating potential is applied for about $2 \mu \mathrm{sec}$ to withdraw the ions, and the cycle is repeated 10,000 times per second. The ion current is detected by an electron multiplier system and can be displayed as a function of drift time on an oscilloscope screen.

The ion current was applied to the vertical deflection plates of a Tektronix Model 545A oscilloscope equipped with a Model 1 Al twochannel preamplifier, whose horizontal sweep was triggered at the end of each accelerating pulse. Thus, the entire mass spectrum from mass 1 to 5000 , or any chosen portion thereof, could be displayed on the oscilloscope screen as ion-current intensity (voltage pulses) versus time of flight. Drift times of ions of interest were measured by comparing them with the residual-gas peaks, hydrocarbon-impurity peaks, or the isotopes of mercury present in the background spectrum. The mercury spectrum arises because a mercury diffusion pump was used to evacuate the system. Determination of the ionic mass followed from Eq. 4.1.

Two counting circuits operating from two auxiliary anodes at the detector can be gated electronically to measure the currents due to ions of any two chosen masses, the current being displayed on the control 
panel meter or recorded. Due to the relatively low sensitivity of the oscilloscope, peaks corresponding to species of low intensity were detected by recording on a Varian Model G-11A potentiometric strip-chart recorder. For recording of fast processes, a Honeywell Model $906 \mathrm{C} \mathrm{Visicorder} \mathrm{os-}$ cillograph with a response frequency of $240 \mathrm{~Hz}$ was used.

Two modifications to the spectrometer developed by Studier ${ }^{141}$ and by Studier and Haumann ${ }^{142}$ are used in this work. The first modification $^{141}$ leads to improved performance of the continuous-ion production mode of operation. The electron-ionizing beam traverses the ion-source region between the backing plate and the firstion-accelerating grid. The positive ions produced are confined by a potential well created by the electron-beam space charge. Every $100 \mu \mathrm{sec}$ the ions are drawn out of the source region and accelerated by application of a negative pulse to the first grid. This pulse destroys the potential well by deflecting the electron beam into the backing plate. To prevent unfocused ions from reaching the flight tube before the well is reformed, pulse-shaping techniques and directcurrent biases are used:

With the electron gun operating continuously, a factor of 300 increase in sensitivity over that of the pulsed mode of operation is attained with improved resolution and no decrease in the signal-to-noise ratio. An experimental sensitivity of $10^{-16}$ Torr:is feasible with this mode of operation. The same pulse-shaping technique can be used for focusing ions from heated-filament, surface-ionization sources.

The second modification ${ }^{142}$ is a blanking generator developed for use with the magnetic electron multiplier in the time-of-flight mass spectrometer. Electrons produced at the first cathode of the multiplier fail to reach the dynode strip for amplification when the potentials of the $\mathrm{Z}$ plate and cathode No. 2 are the same. Applying a $250-\mathrm{V}$ negative pulse of variable width to cathode No. 2 with respect to the $Z$ plate provides a movable window for viewing the desired portion of the spectrum. Blanking out superfluous portions of the mass spectrum minimizes nonlinearity in the electron multiplier, increases gain, and retards dirtying of the dynode strip.

The effect of the blanking circuit is illustrated in Fig. 7. The oscilloscope trace of the mercury spectrum on the left is enhanced with respect to the mercury spectrum on the right where no blanking of other mass peaks was used.

The resolution capabilities of the time- of-flight mass spectrometer are illustrated in Figs. 8 and 9. Figure 8 shows an oscilloscope trace of the isotopes of mercury. Figure 9 is a recorder trace of the same mercury spectrum. The mercury vapor present in the spectrometer ion source comes from the mercury used as the pumping fluid in the diffusion pump. 


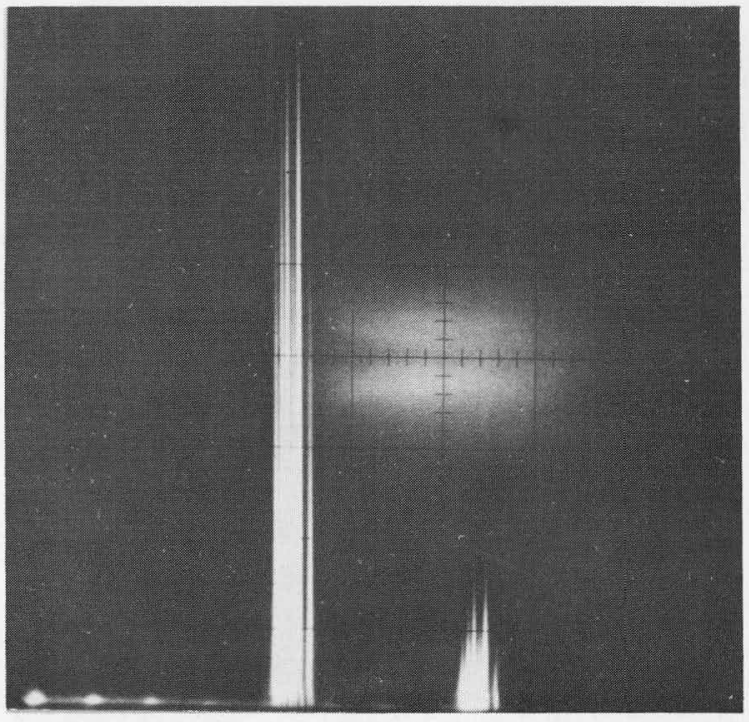

$121-2839$

Fig. 7. Operation of Blanking Generator

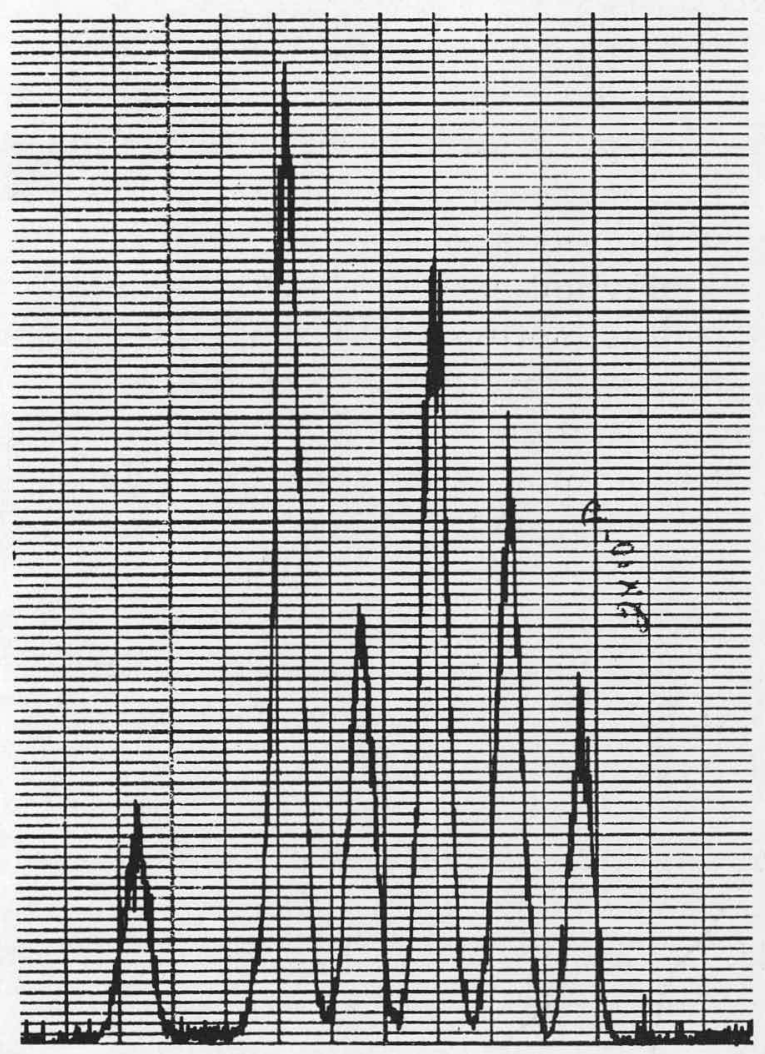

$120-7269$

Fig. 9. Recorder Scan of Mercury with Ion Source Tuned for Maximum Resolution

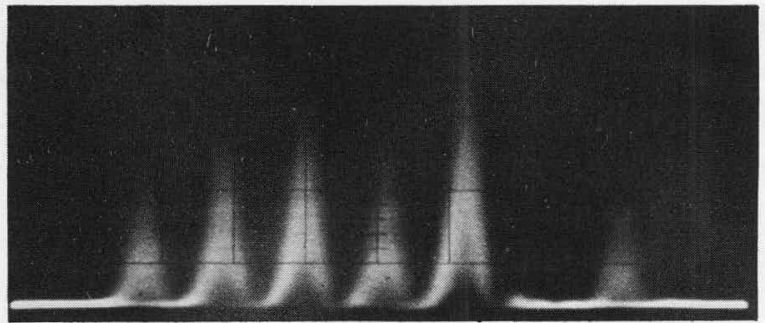

$120-7271$

Fig. 8. Mercury Spectrum

Rhenium filaments $2 \mathrm{~cm}$ long, $0.051 \mathrm{~cm}$ wide, and $1 \mathrm{mil}$ thick were bent into the shape of a square U. The legs, $1 \mathrm{~cm}$ apart, were spotwelded onto 60-mil-diam tungsten rods. The tungsten rods were connected to Kovar feedthroughs, with brass connectors forming a rigid filament support.

The relative positions of the filaments in the ion source are shown (later) in Fig. 21 , a schematic diagram of the arrangement for thermionic work-function measurements. In this diagram, the emitter filament is the sample filament and the collector filament is the surface-ionization filament when surface-ionization measurements are discussed.

The surface-ionization ribbon is placed between the backing plate and the first ion-accelerating grid of the ion source, $1 / 16$ in. below the 0.5-in.-diam hole centered in the first grid. The $1.5 \times 1.5$-sq-in. stainless steel backing plate and the first grid are spaced 0.25 in. apart. 
The sample filament containing the material to be evaporated is situated in the source housing above the ion source proper. Its distance from the surface-ionization filament is $9.05 \mathrm{~cm}$ in a vertical direction normal to the exposed area of the surface-ionization filament. A geometrical area of $0.051 \mathrm{sq} \mathrm{cm}$ of each filament is exposed to the other filament. The distance between the sample filament and the surface-ionization filament is sufficient to preclude radiation heating of the sample by the hot filament. This avoids extraneous evaporation, particularly from samples of relatively high volatility.

The fraction of the flux emitted by the sample filament that is intercepted by the surface-ionization filament is readily calculated for certain geometrical arrangements. Two conditions are required to simplify the calculation: (1) The areas of emission and interception are small with respect to their distance from one another, and (2) the areas are perpendicular to the normal joining their centers. The geometry factor, $G$, is then given by the ratio of the sample-filament area to the product of $\mathrm{pi}$ and the square of the distance between the filaments. For the given geometry, $\mathrm{G}=\mathrm{A}_{\mathrm{S}} / \pi \mathrm{L}^{2}=1.98 \times 10^{-4}$.

A combination adsorption shield and collimating slit of platinum is positioned with the slit $1 \mathrm{~cm}$ from the sample filament, as shown schematically in Fig. 21 (later). The preliminary experiments indicated that a large fraction of the species impinging on the surface-ionization filament came from reevaporation of the species adsorbed onto the walls of the backing plate and first grid. To minimize adsorption on the walls adjacent to the surface-ionization filament, a second collimating slit of stainless steel was installed $1.5 \mathrm{in.} \mathrm{from} \mathrm{the} \mathrm{sample} \mathrm{filament.} \mathrm{The} \mathrm{slit} \mathrm{size} \mathrm{was}$ $0.1 \times 1 \mathrm{sq} \mathrm{cm}$. With this arrangement, the sample beam sees only the surface-ionization filament.

The filaments and slits are optically aligned by sighting through an optical window in the flange supporting the surface-ionization filament. This optical window is also used for optical-pyrometer temperature measurements of the filament.

The electron-ionizing beam has a rectangular cross-sectional area of $0.030 \times 0.200 \mathrm{sq}$ in. whose center is $5 / 16 \mathrm{in}$. above the surfaceionization filament and $0.07 \mathrm{in}$. from the backing plate. The electron beam is directed perpendicular to the sample beam. The neutral species and their molecular complexity in the sample beam can be examined by electron-impact ionization. The presence of the surface-ionization filament does not interfere with the electron-gun mode of operation.

When sample molecules strike the hot surface-ionization filament, a fraction of them are dissociated and ionized. The surfaceionization filament is heated electrically with alternating current. A variable bias can be applied through the center tap of the filament 
transformer to retard electron emission from the surface-ionization filament. This bias, combined with pulse-shaping techniques, permits focusing the surface-produced ions, even though the temperature of the ionizing filament may be as high as $2500^{\circ} \mathrm{K}$.

When the resistance of the variable resistor installed between the backing plate and first grid of the ion source for focusing purposes approached zero ohms, the surface-ionization current increased and then disappeared. This was attributed to an approach of zero electric field between these two electrodes, thereby increasing the fraction of ions collected. The disappearance of the ion current is caused by a defocusing effect. The first grid has a positive potential of about $7 \mathrm{~V}$ in the nonpulsed condition.

The variable resistor between the backing plate and the first grid was removed. A battery-resistor combination was installed to provide a variable bias on the backing plate, independent of the first grid. When the backing-plate bias equaled the positive potential on the first grid, the surface-ionization current was a maximum. This maximum current varied markedly with the bias on the surface-ionization filament. When the filament bias was positive with respect to the backing plate and the first-grid electrodes, the current decreased. The maximum current was obtained when the filament bias was zero to a negative $0.5 \mathrm{~V}$ with respect to the other electrodes. Maintenance of the

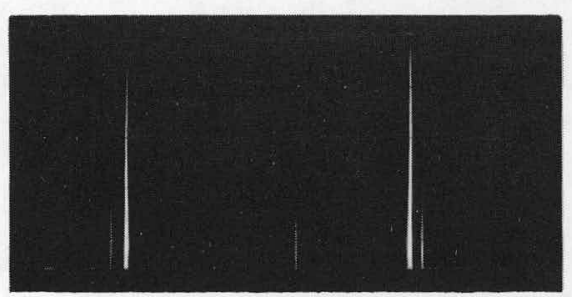

$120-7268$

Fig. 10. Spectra of Alkali Metals $\mathrm{Li}(6,7), \mathrm{Na}(23)$, and K $(39,41)$ from Surface Ionization on Rhenium filament bias at 0.5 to $0.7 \mathrm{~V}$ less than the backing plate and the first grid obviated the necessity for a temperature correction to the surface-ionization current.

Figure 10 is an oscilloscope trace of the spectra of the alkali metals lithium, sodium, and potassium produced by surface ionization from a rhenium filament. The spectrum of sodium and potassium arises from impurity alkali in the rhenium ribbon before complete bakeout.

The heating and flashing controls for the surface-ionization filament is described in Section A.4 of this chapter. Electrical heating of the sample filament is through an ac bridge network. This permits a sufficiently accurate measurement of the sample temperature as shown in Section 3 below. From the temperature and related thermodynamic data, the flux of particles leaving the sample filament can be calculated.

\section{Temperature Measurements}

The four principal methods for measuring temperatures in the range of room temperature to $3000^{\circ} \mathrm{C}$ are optical pyrometry, power 
dissipation, electron flow in a retarding field, and resistance thermometry. The first three methods involve Planck's radiation law, the Stefan-Boltzmann law, and the Boltzmann distribution, respectively. These laws are theoretically valid, and their application has been well established. The procedure that directly measures the velocity distribution of electrons is regarded as absolute. The measurement of temperature by optical pyrometry and power dissipation involves making a number of semiempirical assumptions when using the corresponding theoretical expression.

On the other hand, relationship between the resistance and temperature is empirically derived. For tungsten, Jones and Langmuir ${ }^{143}$ and Forsyth and Worthing ${ }^{144}$ found the empirical relationship

$$
R / R_{0}=\left(T / T_{0}\right)^{1.2}
$$

to hold accurately above $600^{\circ} \mathrm{K}$, where $\mathrm{R}_{0}$ and $\mathrm{T}_{0}$ are the reference resis tance and temperature, respectively, and $\mathrm{R}$ is the resistance at temperature $\mathrm{T}^{\circ} \mathrm{K}$ with $\mathrm{T}>\mathrm{T}_{0}$. This relationship holds only for tungsten filaments of circular cross section. Hill, Lecchini, and Pethica ${ }^{145}$ found that for polycrystalline ribbons of tungsten the value of the exponent is approximately 1.4. They ascribed the differences in the observed behavior between wires and ribbons to lattice defects and dislocations.

These results point out the necessity of a calibration for each ribbon used and voids the use of other calibration tables for calculating the temperature from a measured resistance.

The following sections describe the calibration and measurement of temperatures, using resistance thermometry for the sample filament and the optical-pyrometry method for the hot filament. Resistance thermometry is used below about $800^{\circ} \mathrm{C}$, the lower limit of temperature for optical pyrometry.

a. Resistance Thermometry. The ultimate purpose of resistance thermometry is to obtain the number of atoms or molecules impinging on a hot ribbon some distance away from the source. The source of these molecules is a metal filament containing a sample of the material under investigation. From the thermodynamic properties of the material at a measured temperature, the vapor pressure (and hence the flux of particles arriving at the hot filament) may be obtained.

(1) Calibration of Sample Filament. The resistivity of rhenium varies with the condition of the material and its temperature and is sensitive to purity. In general, cold working increases and annealing decreases the resistivity. For wires of different sizes, the resistance, $\mathrm{R}$, increases with the length and diminishes as the cross-sectional area becomes larger. These facts may be described by the equation 


$$
\mathrm{R}=\rho \frac{l}{\mathrm{~A}},
$$

where $l$ is the length in centimeters, $A$ is the cross-sectional area in square centimeters, and $\rho$ is the resistivity in ohm-centimeters and depends on the material of the conductor.

The resistivity of a substance (and hence the resistance of a wire or ribbon of that substance) depends also upon the temperature. The relationship between the resistance and the temperature may be represented in a purely empirical fashion by a power series of the form

$$
R_{T}=R_{r}\left[1+\alpha\left(T-T_{r}\right)+\beta\left(T-T_{r}\right)^{2}+\ldots\right] \text {, }
$$

where $R_{T}$ is the resistance at the temperature $T^{\circ} K$, and $R_{r}$ is the resistance at the temperature of reference, $\operatorname{Tr}$. The numerical values of the coefficients depend upon the reference temperature. In general, for a three-term series, the constants $\alpha$ and $\beta$ are determined from the values of $R_{T}$ at three reproducible temperatures to which a fixed numerical value is assigned based on the International Practical Temperature Scale. ${ }^{146}$

In these experiments, however, high sample-filament temperatures were determined by an accurately calibrated optical pyrometer, and the corresponding resistance values were measured. A roomtemperature reference value and the corresponding reference resistance were also measured using an accurate standard glass thermometer. The coefficients, $\alpha$ and $\beta$ in Eq. 4.4 were then calculated using the room temperature and two selected high temperatures as fixed points. The errors assigned to the coefficients were determined from the errors in the readings of the bridge resistance values.

The fixed points are not exactly fixed, but have also an error associated with the reading of the temperature by optical pyrometry. Under these conditions, the errors assigned to the temperature coefficients were calculated from the errors in both the resistance and temperature values. The results are shown in Table VI.

TABLE VI. Values and Errors of the Temperature Coefficients in the Power Series Relating Temperature to Resistance

\begin{tabular}{llc}
\hline & \multicolumn{2}{c}{ Standard Error } \\
\cline { 2 - 3 } Temperature Coefficients & Fixed $\mathrm{T}$ & Error in T \\
\hline$\alpha=2.817 \times 10^{-3}{ }^{\circ} \mathrm{K}^{-1}$ & 0.010 & 0.039 \\
$\beta=-5.45 \times 10^{-7}{ }^{\circ} \mathrm{K}^{-2}$ & 0.10 & 0.41 \\
\hline
\end{tabular}

a The temperatures used in determining the numerical values of the temperature coefficients were $294.3 \pm 0.1,1155 \pm 2$, and $1296 \pm 2^{\circ} \mathrm{K}$, with bridge-resistance values of $5.70 \pm 0.005,17.22 \pm$ 0.005 , and $18.67 \pm 0.005 \mathrm{ohms}$, respectively. 
A calibration curve of bridge resistance versus temperature was then constructed, based on the values of the temperature coefficients and the reference resistance value. From Eq. 4.4, letting $\mathrm{T}-\mathrm{T}_{\mathrm{r}}=\Delta \mathrm{T}$ and $\left(\mathrm{R}_{\mathrm{T}}-\mathrm{R}_{\mathrm{r}}\right) / \mathrm{R}_{\mathrm{r}}=\mathrm{K}$,

$$
\Delta \mathrm{T}=\frac{-\alpha+\sqrt{\alpha^{2}+4 \beta \mathrm{K}}}{2 \beta}=(2.5845-\sqrt{6.680-1.835 \mathrm{~K}}) 10^{3} .
$$

The calculated values of the temperature obtained for various values of bridge resistance are shown in Table VII. The calibration curve (with the estimated limits of error) is shown in Fig. 11.

TABLE VII. Temperature vs Bridge Resistance

\begin{tabular}{|c|c|c|c|c|}
\hline $\mathrm{R}_{\mathrm{T}}$, ohms & $\mathrm{K}$ & $\Delta \mathrm{T},{ }^{\circ} \mathrm{K}$ & $\mathrm{T},{ }^{\circ} \mathrm{K}$, calc & $\mathrm{T},{ }^{\circ} \mathrm{K}$, measured ${ }^{\mathrm{a}}$ \\
\hline 19.35 & 2.395 & 1073 & 1367 & 1365 \\
\hline $18.67^{\mathrm{b}}$ & 2.275 & 1002 & 1296 & 12.96 \\
\hline 18.38 & 2.225 & 973 & 1267 & 1269 \\
\hline 18.00 & 2.158 & 936 & 1230 & 1230 \\
\hline 17.78 & 2.119 & 914 & 1208 & 1210 \\
\hline $17.22^{b}$ & 2.021 & 861 & 1155 & 1155 \\
\hline 17.00 & 1.982 & 841 & 1135 & \\
\hline 16.00 & 1.807 & 751 & 1045 & \\
\hline 15.00 & 1.632 & 665 & 959 & \\
\hline 14.00 & 1.456 & 583 & 877 & \\
\hline 13.00 & 1.281 & 504 & 798 & \\
\hline 12.00 & 1.105 & 428 & 722 & \\
\hline 11.00 & 0.930 & 355 & 649 & \\
\hline 10.00 & 0.754 & 284 & 578 & \\
\hline 9.00 & 0.579 & 215 & 509 & \\
\hline 8.00 & 0.404 & 148 & 442 & \\
\hline 7.00 & 0.228 & 83 & 377 & \\
\hline 6.00 & 0.053 & 19 & 313 & \\
\hline $5.70 \mathrm{~b}$ & & & & 294.3 \\
\hline
\end{tabular}

${ }^{a}$ By optical pyrometry.

b Calibration point. 


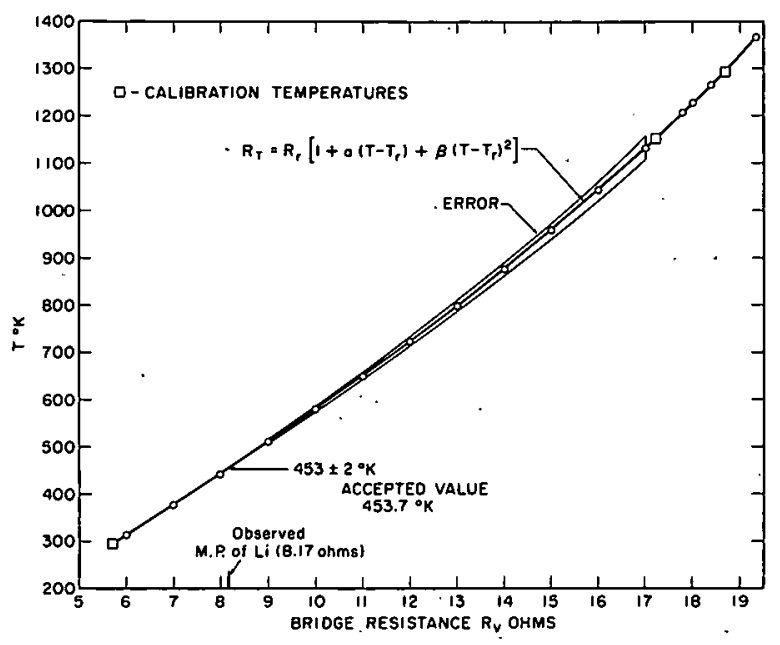

$121-5491$

Fig. 11. Temperature vs Bridge Resistance
In general, an error of $1 \%$ in the temperature will contribute little to the error in the determination of the vapor pressure, since the error in the heat of sublimation or vaporization is greater than this.

The suitability of the threeterm power-series equation relating temperature to bridge-resistance values was checked by measuring the bridge resistance at the point where a sample of lithium metal on the sample filament was observed to melt. The sample size was about $10 \mu \mathrm{g}$ and covered approximately the center half of the effective area

of the sample filament. Using focused magnifying optics permitted the point of melting to be observed. From the measured resistance value, the corresponding temperature obtained was compared with the melting point of lithium, $453.7^{\circ} \mathrm{K} .{ }^{100}$ These results also are shown in Fig. 11.

The room-temperature reference value for the resistance of rhenium may be compared with published values. Sims, Craighead, and Jaffee ${ }^{147}$. determined the resistivity of annealed rhenium wire to be $\rho=19.14 \pm 0.02 \times 10^{-6} \mathrm{ohm}-\mathrm{cm}$. Caldwel1 ${ }^{148}$ gave a table of values for the resistivity of rhenium wire in circular mil ohms per foot (ohms/cmf) at various temperatures, reproduced in Table VIII. These values were converted to resistivity in ohm-centimeters by multiplying by $1.6624 \mathrm{x}$ $10^{-7}$ ohm-cm per circular mil ohm per foot.

TABLE VIII. Resistivity of Rhenium, from Caldwel1 ${ }^{140}$

\begin{tabular}{|c|c|c|c|c|c|}
\hline $\mathrm{t},{ }^{\circ} \mathrm{C}$ & $\rho$, ohms/cmf & $\rho \times 10^{6}$, ohm-cm & $t,{ }^{\circ} \mathrm{C}$ & $\rho$, ohms/cmf & $\rho \times 10^{6}$, ohm-cm \\
\hline 20 & 116 & 19.28 & 800 & 403 & 66.99 \\
\hline 200 & 197 & 32.75 & 1000 & 460 & 76.47 \\
\hline 400 & 280 & 46.55 & 1200 & 505 & 83.95 \\
\hline 600 & 349 & 58.02 & & & \\
\hline
\end{tabular}

For a rhenium ribbon $2 \mathrm{~cm}$ long and of $0.02 \mathrm{x}$ $0.001-\mathrm{sq}$-in. cross section,

$$
R=1.55 \times 10^{4} \rho \text { ohms. }
$$

If $19.28 \times 10^{-6} \mathrm{ohm}-\mathrm{cm}$ is used for the resistivity of rhenium at room temperature, the resistance of the filament, $R_{f}$, will be 
0.299 ohm.. Multiplication by the bridge ratio gives a value of 6.05 ohms. The measured bridge resistance for a rhenium ribbon of this approximate size was $5.70 \mathrm{ohms}$, which includes a nearly negligible contribution from the copper leads amounting to $0.002 \mathrm{ohm}$ or a bridge-resistance value of $0.04 \mathrm{ohm}$. (The copper leads consisted of $2 \mathrm{ft}$ of $\mathrm{B}+\mathrm{S}$ Gauge No. 10 copper wire with a resistance of $0.001 \mathrm{ohm} / \mathrm{ft}$.)

The rhenium ribbons are welded to thick tungsten feedthroughs. As the filament is heated, a temperature gradient exists between the tungsten binding posts and the portion of the ribbon near the posts. Since the gradient changes with temperature, this precludes the use of any established tables of resistance versus temperature, but does not inviolate a direct calibration. No detectable temperature gradient was observed in the center half of the rhenium ribbon at high temperatures.

The measured resistance, and hence the temperature of the effective portion of the rhenium ribbon, is assumed to be unaffected when a salt or another metal is adsorbed or placed on the ribbon. Suhrmann ${ }^{149}$ showed that a change in resistance of thin metal films due to adsorbed gases will be of measurable magnitude, provided the adsorbing layer is not thicker than 100 to 1000 times the thickness of the adsorbed layer undergoing electronic interaction. The thickness of the adsorbed layer undergoing electronic interaction may be estimated from the following considerations.

The adsorption of alkali metal atoms on metallic surfaces lowers the electron work function of the adsorbing metal surface on account of the high polarizability of the atoms. With increasing coverage, the work function decreases to a minimum somewhat below that for monatomic covering, $\theta=1$. Then the work function increases to a value for the pure foreign metal itself. This is reached when about five atom layers of the adsorbed metal have been deposited, ${ }^{150}$ indicating that further increasing the adsorbed film thickness leads to no further electronic interaction. Using a radius of $1 \AA$ for lithium (halfway between the ionic and metallic radii of 0.6 and $1.52 \AA$, respectively) yields an adsorbed thickness of approximately $10 \AA$.

To obtain a measurable change in resistance of the order of $1 \%$ requires an adsorbent metal film thickness of no more than $10^{-4} \mathrm{~cm}$. The actual rhenium metal ribbon thickness is about $1 \mathrm{mil}$ or $2.5 \times 10^{-3} \mathrm{~cm}$. The corresponding change in the resistance of the rhenium ribbon will be less than the error inherent in the measuring system used. This agrees' with the results obtained in the temperature measurement of the melting point of lithium described on page 69. For salts deposited on the rhenium ribbon, the electronic interaction is decreased, due to the smaller effective thickness of the salt layer. 
Table VIII and Eq. 4.6 suggest that a measuring system capable of detecting a change of about 0.001 ohm per degree in the filament resistance over a range of $1000^{\circ}$ is required. Such a system is described in Section (2) below.

\section{(2) Bridge Circuitry}

(a) Introduction. Various methods for measuring resistances by bridge circuitry are available. ${ }^{151,152}$ Alternating current bridge methods for measuring thermometer resistance have certain basic advantages. One of these is the elimination of the influence of thermoelectric emfs, since these do not affect an ac null-detection system. Another advantage is the practicability of setting up and maintaining very precise voltage ratios with inductive devices whose parameters can be calculated. In effect, a more sensitive ac amplifier can be made more easily than a dc amplifier, because there is not the annoying.problem of "zero drift" to be overcome.

These advantages are available at a premium, however. If the resistance leads are long and/or coiled, inductive effects are certain to be present. A resistance standard is required for comparison, and the ac properties of this element are critical. Stray capacitance of the transformers coupling a bridge to a detector and/or power supply must be controlled. It may be necessary to provide some means of reducing the effects of stray impedance between the bridge elements and ground.

If there is reactance in one of the bridge arms (e.g., in the unknown element), there is no adjustment of the standard resistance that will yield a true null across the bridge. This is because the reactance in the unknown causes a phase shift with respect to the standard side of the bridge which cannot be balanced out by the adjustment of pure resistances. A minimum will be observed rather than a null, but the minimum does not occur at the same setting of the standard that the purely resistive unknown would give. To find the true value of the resistance in a.resistive-reactive component, the reactive part of the impedance, as well as the resistive, must be balanced.

The oscilloscope rotating-trace method of null indication in ac bridges for measuring complex impedances has a.definite advantage ${ }^{153}$. One essential point is that the horizontal deflection voltage need not necessarily originate directly from the bridge diagonal, but can be obtained from the voltage source in other ways. Phase shifts occur so that the pattern.on the oscilloscope screen is no longer a simple trace but a more or less open ellipse. The vertical amplifier may also cause a phase shift. If considerable phase correction is necessary, antiphaseshifting networks can be incorporated simultaneously for both the $\mathrm{x}$ and $y$. deflection. 
For example; RC phase-shifting networks. in the out-of-balance voltage: lead to the vertical amplifier and in the deflectionvoltage lead to the horizontal amplifier may be used. The output voltage across the resistor in the RC network leads that of the input voltage. The output voltage across the capacitor lags behind the input voltage to the RC network.

The networks must be so rated that, when comparing impedances without phase difference, a simple trace will again appear on the screen. According to the ratio $\mathrm{R}: 1 / \omega \mathrm{C}$, the phase shift, when $R=1 / \omega C$, will be $45^{\circ}$. The phase difference obtainable with both networks will be $9.0^{\circ}$. At the same time, the voltage at the output of each network will fall to $\sim 0.7$. of its input value. Once the correction has been made, complex impedances in bridge circuits can be measured as follows.

Since the out-of-balance voltage is complex, it consists of two voltage vectors corresponding to the different types of impedances. The pattern on the oscilloscope screen when the bridge is off balance will not be a simple trace, but an ellipse. If the variableresistance arm of the bridge is operated, the resistive bridge balance can be restored. The ellipse rotates on the screento a position with the foci on the horizontal axis. But beçause of the reactant component in the bridge circuit, there will still be a residual voltage to deal with. Since the vector of this voltage is at right angles. to the resistive voltage, the ellipse will remain open, even when the bridge is resistively balanced. Only, when the reactive component is adjusted to a null value will the ellipse collapse to the null line.

Note that the "bridge balance" and "phase balance" are now, in principle, completely. independent of each other. In this procedure, the influence of each balance can be seen separately. Thus, when the bridge is unbalanced, the phase balance may first be adjusted to collapse the ellipse, and then the resistive component of the bridge can be balanced to rotate the line to a horizontal null.

This is a unique advantage compared with all other methods used.for measurements of this kind, which always require alternate adjustments of bridge balance and phase balance until a minimum is reached.

The ac bridge employed in this work for the measurement of resistance and hence of the temperature uses the principles of the Maxwell bridge. ${ }^{154}$ Figure 12 shows the circuitry used with the associated power supply and null detection. 


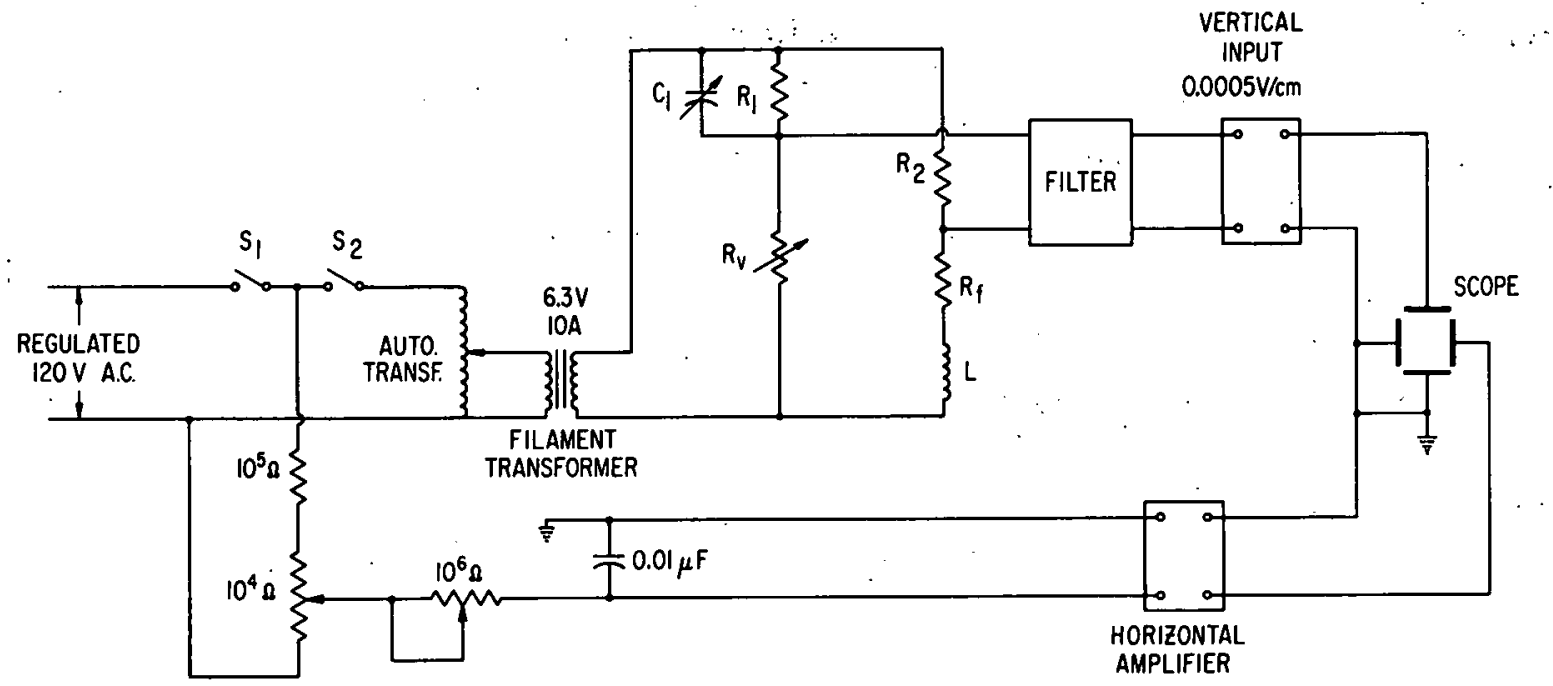

$121-5498$

Fig. 12. Maxwell Bridge and Associated Null-detection Circuitry

(b) Mathematical Analysis of the Maxwell Bridge.

The current $I_{1}$ flows through the fixed resistor, $R_{1}$, and the variable decade resistor, $R_{V}$; the current $I_{2}$ flows through the fixed resistor $R_{2}$ and the filament resistance $R_{f}$. When the bridge is balanced,

$$
\overline{\mathrm{I}}_{1} \overline{\mathrm{Z}}_{1}=\overline{\mathrm{I}}_{2} \overline{\mathrm{Z}}_{2}
$$

and

$$
\bar{I}_{1} \bar{Z}_{v}=\bar{I}_{2} \bar{Z}_{f}
$$

Therefore

$$
\overline{\mathrm{z}}_{\mathrm{f}}=\frac{\overline{\mathrm{z}}_{2} \overline{\mathrm{Z}}_{\mathrm{v}}}{\overline{\mathrm{Z}}_{1}}
$$

where $\bar{Z}$ is the vector impedance of the bridge arms. The impedance $\bar{Z}_{1}$ is composed of a parallel combination of resistance $R_{1}$ and capacitance $\mathrm{C}_{1}$. The admittance $\overline{\mathrm{Y}}_{1}$ of this combination is given by

$$
\overline{\mathrm{Y}}_{1}=\frac{1}{\overline{\mathrm{Z}}_{1}}=\frac{1}{\mathrm{R}_{1}}+\frac{1}{\overline{\mathrm{X}}_{\mathrm{C}_{1}}}
$$

where $\overline{\mathrm{X}}_{\mathrm{C}_{1}}$ is the vector reactance.

In complex notation,

$$
\bar{X}_{C_{1}}=-j X_{C_{1}}=-\frac{j}{\omega C_{1}},
$$


where $j=\sqrt{-1}$, and the admittance is now represented as

$$
\bar{Y}_{1}=\frac{1}{R_{1}}+j \omega C_{1}
$$

and from Eq. 4.9,

$$
\bar{Z}_{f}=R_{2} R_{v}\left(\frac{1}{R_{1}}+j \omega C_{i}\right)
$$

The vector impedance of the filament arm of the

bridge is given also by

$$
\bar{Z}_{f}=R_{f}+\bar{X}_{L}=R_{f}+j X_{L}=R_{f}+j \omega L
$$

where $X_{L}$ is the inductive reactance, and $L$ is the inductance induced in the filament arm of the bridge on passing a current through it. If Eqs. 4.13 and 4.14 are equated, the resistive condition of balance is

$$
R_{f}=\frac{R_{2} R_{Y}}{R_{1}}
$$

and the inductive condition of balance is

$$
L=R_{2} R_{V} C_{1}
$$

Notice that the conditions of balance do not contain a frequency term. Theoretically, then, this type of bridge could be operated from a generator of any waveform, or even from pulses or switching transients. In the Maxwell bridge, balancing the resistive component of the bridge also acts in a major way to balance the inductive component of the bridge. The final balance of the inductive component can then be accomplished with a suitable capacitor value:

So far, only sinusoidal voltage measurements have been considered. Occasionally measurements have to be made with voltages containing harmonics, e.g., using a test voltage taken directly from the mains. without filtering. Measurements are still possible here, whereas with other methods of null indication no satisfactory results could be produced. Moreover, in distortion measurements using bridge circuits, the harmonics can thus be separated from the fundamentals.

(c) Bridge Sensitivity. The minimum deflection coefficient of the available oscilloscope is $0.5 \mathrm{mV} / \mathrm{cm}$, and the mean diameter of the trace is $0.5 \mathrm{~mm}$. A change in the position of the extremity of the null indication due to an out-of-balance voltage of $0.025 \mathrm{mV}$ is still readable on the scireen. 
The decade resistance arm of the bridge can be set to within $\pm 0.005 \mathrm{ohm}$ of the null condition. Table IX gives, for an essentially infinite load resistance, the maximum out-of-balance voltage, $E_{L}$, obtained for an imbalance of \pm 0.005 ohm in $R_{V}$, at three values of $R_{V}$. The bridge input peak-to-peak voltages, $E_{p p}$, were determined from oscilloscope measurements. The calculated results indicate a detection system of sufficient sensitivity.

TABLE IX. Out-of-balance Voltage Expected for an Imbalance of $\pm 0.005 \mathrm{ohm}$ in $R_{V}$ at Various Values of $R_{V}$

\begin{tabular}{rrrr}
\hline $\mathrm{T},{ }^{\circ} \mathrm{K}$ & $\mathrm{R}_{\mathrm{V}}$, ohms & $\mathrm{E}_{\mathrm{pp}}, \mathrm{V}$ & $\mathrm{E}_{\mathrm{L}, \mathrm{mV}}$ \\
\hline 441 & 8.00 & 0.336 & 0.050 \\
880 & 13.00 & 1.45 & 0.140 \\
1180 & 17.50 & 2.45 & 0.160 \\
\hline
\end{tabular}

(d) Illustration of Null Detection. The horizontal deflection factor of the oscilloscope was continuously variable from 0.2 to $15 \mathrm{~V} / \mathrm{cm}$ and could be phase-shifted. The vertical-input deflection factor was $0.5 \mathrm{mV} / \mathrm{cm}$. Figure 13 shows an unbalanced and a null condition of the bridge. At relatively high temperatures, the $180-\mathrm{Hz}$ source becomes

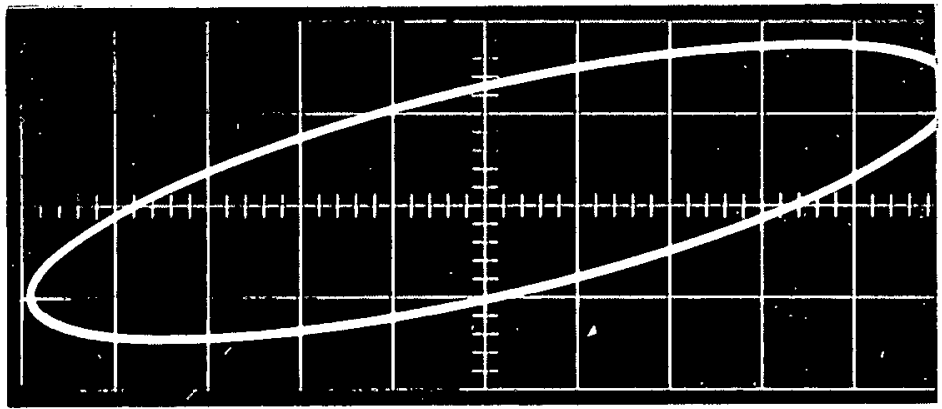

a

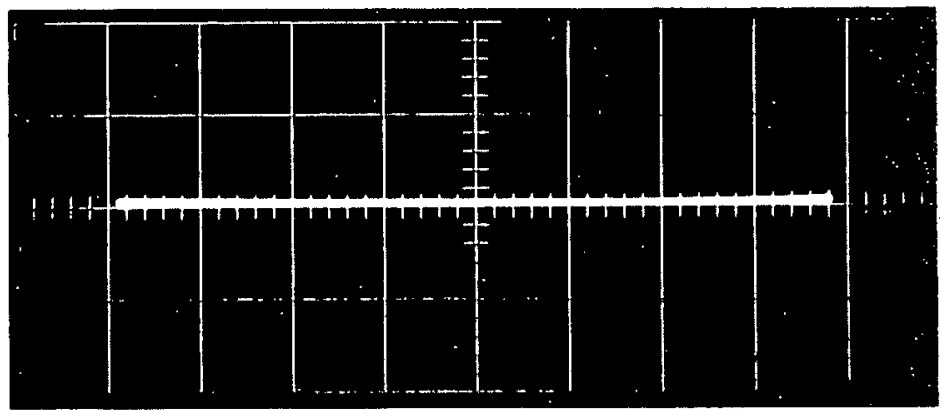

b

$121-5492$

Fig. 13. Illustration of Bridge Balancing; a. Unbalanced and b. At null 
pronounced. This tends to distort the null position. However, varying the phase of the horizontal deflection voltage enables a convenient display to be obtained. This is illustrated in the Lissajous figure of Fig. 14 which shows a frequency ratio of 3:1 for the vertical deflected voltage frequency to the horizontal deflected voltage frequency at a phase difference of $90^{\circ}$, with the $60-\mathrm{Hz}$ vertical component nulled out.

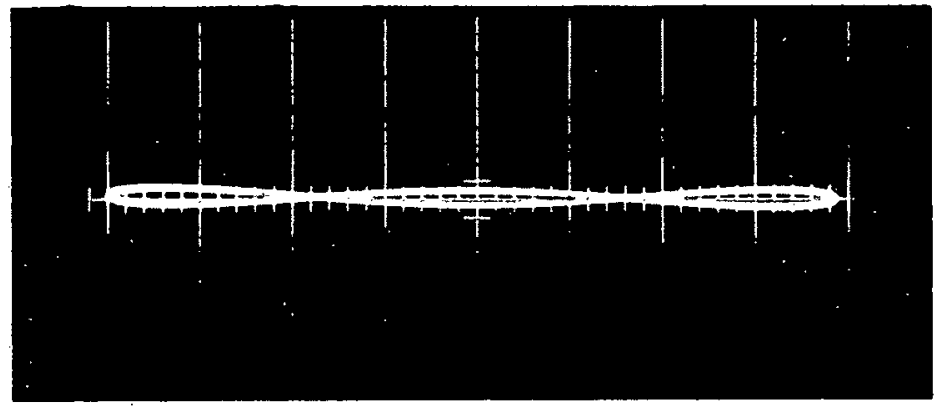

$121-5486$

Fig. 14. Bridge Null Condition with a $180-\mathrm{Hz}$ Harmonic Present

(e) Calibration of Resistors. In addition to the errors in the null-point determination, discussed in Section (d) above, other sources of errors are (1) inaccuracies in the standard and variable resistances, and (2) the change in resistance values with time. The power loss in the various resistors must be kept at a safe value.

At the null condition of Fig. 13,

$$
R_{f}=R_{V} \frac{R_{2}}{R_{1}}
$$

where $R_{f}$ is the rhenium ribbon resistance, $R_{1}$ and $R_{2}$ are the standard resistances, and $R_{V}$ is the variable-resistance arm of the bridge. A precision resistor of $1 \mathrm{ohm}$, accurate to $0.002 \%$ was used to check a Model RN-1 Industrial Instrument Company Wheatstone bridge for accuracy. Resistor $R_{1}$, a 10-ohm precision resistor, was measured and found to have a value of $10.010 \pm 0.005$ ohms. Resistor $R_{2}$, consisting of five 0.1 -ohm precision. resistors mounted on a copper block, measured $0.4945 \pm$ $0.0005 \mathrm{ohm}$. The resistance ratio, $\mathrm{R}_{2} / \mathrm{R}_{1}$, therefore, has a value of $0.0494 \pm 0.00005$, an accuracy of $0.1 \%$.

The variable resistor, $R_{v}$, is a Hallcross No. 817-B Decade Resistance Box, graduated in steps of 0.01 to $1111.1 \mathrm{ohms.A}$ calibration curve of the variable resistance setting to the measured value as determined by the Wheatstone bridge was obtained. The dial settings were low by a maximum of $0.5 \%$, the calibrated values of the dial setting being accurate to within $0.05 \%$. Under these conditions, the errors in the determination of the temperatures are but little affected by the errors in the resistances. 
The variable capacitor is an Industrial Instrument Company Decade Capacitor Box, graduated in steps of 0.001 up to $1.111 \mu \mathrm{F}$, and was used in parallel with a fixed capacitor of $1 \mu \mathrm{F}$.

b. Optical Pyrometry. In the study of the surface ionization of a beam of particles striking a hot filament, the variation in the ions produced as a function of temperature is required. The temperature of incandescent bodies can be measured with a dissappearing-filament optical pyrometer. Only a brief description of the procedure for calibrating an optical pyrometer is given. For a more detailed discussion, several sources are available. $155=157$

(1) Calibration. Due to differences in eye sensitivity among humans, each user must perform his own calibration to obtain the ultimate precision possible with the instrument. A Leeds and Northrup disappearing-filament optical pyrometer (Model 8622C, Serial No. 778054), the freezing temperature of copper, a blackbody, and rotating sectored discs were used to realize the temperature scale from about 800 to $2500^{\circ} \mathrm{C}$.

With a blackbody immersed in copper at its freezing temperature $\left(1083^{\circ} \mathrm{C}\right)$, the electrical current through the tungsten filament of the pyrometer is adjusted until the filament image "disappears" against the background of the blackbody. This establishes one fixed point or reference temperature, which may be expressed in terms of a current or a position on the scale of the pyrometer, from which other temperatures may be determined. The actual fixed point was established by taking the average of several readings of the freezing-point plateau in the cooling curve for copper.

The blackbody is next heated to some unknown temperature, $T_{1}$, such that when measured through a rotating sectored disc of transmissivity $\tau$, by rematching the filament, $T_{1}$ is the fixed point within a negligible small deviation. The rotating sectored disc is now removed, and the current through the pyrometer filament is readjusted to another match, with the blackbody remaining at $T_{1}$. This reading is then compared with the true temperature calculated from the relationship.

$$
\frac{1}{\mathrm{~T}_{1}}-\frac{1}{\mathrm{~T}}=\frac{\lambda_{\mathrm{e}}}{\mathrm{C}_{2}} \ln \tau,
$$

where $\mathrm{T}$ is the reference temperature as measured with the sectored disc, $\lambda_{e}$ is the effective wavelength of the transmitted light, $\tau$ is the transmissivity of the sectored disc, and $\mathrm{C}_{2}$ is the second radiation constant, equal to $1.4380 \mathrm{~cm}$ per degree. The true temperature, $\mathrm{T}_{1}$, is calculated from Eq. 4.17 to which the temperature, as measured without the sectored disc, is to be compared. This establishes the second point, $T_{1}$, on the temperature or pyrometer scale. The: procedure is repeated using rotating sectored discs of different transmissivities at other blackbody temperatures 
to establish the $\mathrm{L}$ scale of the pyrometer. The $\mathrm{H}$ scale is then calibrated from the $L$ scale and the $\mathrm{xH}$ scale from the $\mathrm{H}$ scale. In all instances, the pyrometer readings were the average of five determinations.

The form of Eq. 4.17 is based on the following considerations: The functional form of Planck's equation expressing the flux density of radiation of a blackbody is given by

$$
J(\lambda, t)=\frac{C_{1}}{\lambda^{5}\left[\exp \left(\frac{C_{2}}{\lambda T}\right)-1\right]},
$$

where $J(\lambda, T)$ is the flux density at the wavelength $\lambda$ and temperature $T$, $\mathrm{C}_{1}(=8 \pi \mathrm{hc})$ is the first radiation constant, and $\mathrm{C}_{2}(=\mathrm{hc} / \mathrm{k})$ is the second. The symbols $h, c$, and $k$ are Planck's constant, the velocity of light, and Boltzmann's constant, respectively.

When a match is obtained, the integral luminosity at the eye of the image of the blackbody is equal to that of the filament. Under these conditions, the matching equation is 157

$$
\int \mathrm{V}(\lambda) \operatorname{IF}(\lambda) \operatorname{OJ}(\lambda, T) \mathrm{d} \lambda=\int \mathrm{V}(\lambda) \operatorname{IF}(\lambda) \mathrm{E}\left(\lambda, \mathrm{S}_{0}\right) \mathrm{d} \lambda
$$

where $V(\lambda)$ is the transmissivity of the eye and $I, O$, and $F(\lambda)$ are the transmissivity of the eyepiece, the objective lens, and the red filter, respectively. The symbol $E$ is the flux density of the filament at the fixed point $S_{0}$, and $\lambda$ is the wavelength of the transmitted light. At the blackbody temperature $\mathrm{T}_{1}$ observed through a rotating sectored disc of transmissivity $\tau$, the pyrometer filament is again at the fixed $S_{0}$, within a small deviation, $\delta$. For this match,

$$
\tau \int \mathrm{V}(\lambda) \operatorname{IF}(\lambda) O J\left(\lambda, \mathrm{T}_{1}\right) \mathrm{d} \lambda=\int \mathrm{V}(\lambda) \operatorname{IF}(\lambda) \mathrm{E}\left(\lambda, \mathrm{S}_{0}+\delta\right) \mathrm{d} \lambda
$$

For a negligible deviation, $\delta$, and since $I$ and $O$ are independent of wavelength,

$$
\int \mathrm{V}(\lambda) F(\lambda) \mathrm{J}(\lambda, \mathrm{T}) \mathrm{d} \lambda=\tau \int \mathrm{V}(\lambda) \mathrm{F}(\lambda) \mathrm{J}\left(\lambda, \mathrm{T}_{1}\right) \mathrm{d} \lambda(\delta \rightarrow O)
$$

In practice, it is simpler and adequate below $3000^{\circ} \mathrm{K}$ to use Wien's equation and to define an effective wavelength $\lambda_{e}$ such that

$$
\frac{\int V(\lambda) F(\lambda) J(\lambda, T) d \lambda}{\int V(\lambda) F(\lambda) J\left(\lambda, T_{1}\right) d \lambda}=\exp \left[-\frac{C_{2}}{\lambda_{e}}\left(\frac{1}{T}-\frac{1}{T_{1}}\right)\right]
$$

From this definition, Eq. 4.21 can be written in the form of Eq. 4.17.

The effective wavelength, $\lambda_{e}\left(T, T_{1}\right)$, of the transmitted light defined by Eq. 4.22 may be obtained for any arbitrarily selected 
temperatures. However, this is cumbersome, and the situation can be simplified by calculating values of the effective wavelength as the two temperatures approach each other. A convenient relationship ${ }^{155-157}$ defining a limiting effective wavelength, $\lambda_{\ell}$, is

$$
\lambda_{\mathrm{e}}\left(\frac{1}{\mathrm{~T}}, \cdots \frac{1}{\mathrm{~T}_{1}}\right)=\lambda_{\ell \mathrm{e}}\left[\frac{1}{2}\left(\frac{1}{\mathrm{~T}_{1}}+\frac{1}{\mathrm{~T}}\right)\right] .
$$

The values. for the limiting effective wavelength were arrived at in the following manner: The transmissivities of the red filter and the range screens were determined on a Cary Model 14 Spectrophotometer over a wavelength region of 600-700 $\mathrm{m} \mu$. The wavelength scale of the spectrophotometer was standardized against the spectral lines of mercury. With these data and the eye sensitivity values as given by Judd ${ }^{158}$ for the fully light-adapted eye, the limiting effective wavelengths versus temperature for all three ranges of the optical pyrometer were computed.

This was easily accomplished using a FORTRAN program written for the IBM 1620 computer. The machine integrations were done at wavelength intervals of $2.5 \mathrm{~m} \mu$ over small temperature intervals. The results gave the limiting effective wavelength corresponding to the reciprocal temperature that is the mean of the reciprocal of the high and low temperatures. The effective wavelength is then obtained directly from a curve relating the limiting effective wavelength to the mean of the reciprocal of the high and low temperatures.

The red filter inserted between the filament and the eyepiece of the pyrometer transmits only for wavelengths above about $650 \mathrm{~m} \mu$. Since the spectral sensitivity of the human eye decreases rapidly in the region where the filter transmissivity increases rapidly, the flux densities observed are confined to a rather narrow interval of wavelengths. Under these conditions, the small variation in the emissivity of the tungsten filament does not cause its color to be significantly different from that of the blackbody.

(2) Corrections. The temperature of a rhenium filament in the vacuum system of the mass spectrometer had to be measured. A correction due to the adsorption of radiation by the optical system used to view the rhenium filament was required. A further correction for the emissivity of the rhenium ribbon was also required, since the ribbon is not a blackbody radiator. These corrections to the observed temperatures were determined as follows.

The corrections for the transmissivity of the optical window-prism combination were obtained by measuring the same blackbody temperature with and without the combination. The correction factor, A, was determined from the relationship 


$$
\frac{1}{\mathrm{~T}}-\frac{1}{\mathrm{~T}_{\mathrm{A}}}=\frac{\lambda_{\mathrm{e}}}{\mathrm{C}_{2}} \ln T=-\mathrm{A}
$$

where $\tau$ is the transmissivity of the window-prism combination, $T$ is the true temperature, and $\mathrm{T}_{\mathrm{A}}$ is the apparent temperature when viewed through the combination: The constant, A, was determined to be $(1.633 \pm$ $0.087) 10^{-5}$ per degree Kelvin. This value was used throughout the investigation to make corrections for the transmissivity of the window and the prism. A shutter was used, when appropriate, between the ribbon and the window to be sure that the window did not become coated with condensed rhenium or other vapors and thus change its' radiation adsorption characteristics.

Marple ${ }^{159}$ determined the spectral emissivity of rhenium at a wavelength of $0.65 \mu$. The emis sivity varied from 0.417 to 0.395 over a range of 1537 to $2772^{\circ} \mathrm{C}$. For an emissivity of $\in(\lambda, T)$, the true temperature may be determined from

$$
\frac{1}{T}-\frac{1}{T_{A}}=\frac{\lambda}{C_{2}} \ln [\epsilon(\lambda, T)]
$$

where the symbols have been previously defined.

The emissivity data were extrapolated to $800^{\circ} \mathrm{C}$. A small $(1 \%)$ correction was made to convert the wavelength to the effective wavelength. A standard deviation of \pm 0.015 in the emissivity was used throughout, based on the error analysis of Marple. ${ }^{159}$ The data were replotted to give values of the emissivities at the effective wavelength for arbitrary apparent temperatures.

The total corrections to be applied to the pyrometer readings to obtain the true temperature consisted of the following steps:

1. Pyrometer-reading correction for blackbody calibration.

2. Adsorption correction for window-prism combination.

3. Emissivity correction at temperature determined after step 2 .

For ease of obtaining the true temperature, the observed pyrometer reading was then plotted against the true temperature. An error analysis by means of the law of propagation of errors ${ }^{160}$ was made on the true temperature. The standard deviation in the true temperature amounts to \pm 2 and $\pm 18^{\circ} \mathrm{K}$ at 1100 and $2600^{\circ} \mathrm{K}$, respectively. 
4. Flash-desorption Circuitry

Figure 15 shows the circuitry used to flash the surfaceionization filament to a high temperature automatically. When the threeway switch, $S_{3}$, is in position 1 , timer $T_{1}$ is running and the temperature of the filament is controlled by the setting of variable transformer 1 . When this timer has run its course, switch $S_{1}$ is closed actuating relays $\mathrm{K}_{3}$ and $\mathrm{K}_{1}$. Switch $\mathrm{K}_{3 \mathrm{~B}}$ starts timer $\mathrm{T}_{2}$. Switch $\mathrm{K}_{1} \mathrm{C}$ resets timer $\mathrm{T}_{1}$ to zero opening switch $S_{1}$. At the same time, the filament current is controlled by the setting of variable transformer 2, usually the flashing current. Timer $T_{2}$ controls the duration of the flash. When timer $T_{2}$ has run its course, switch $S_{2}$ is opened, relays $K_{1}$ and $K_{3}$ are deactuated, timer $T_{2}$ is reset to zero, closing switch $S_{2}$, and the first timer begins the cycle over again.
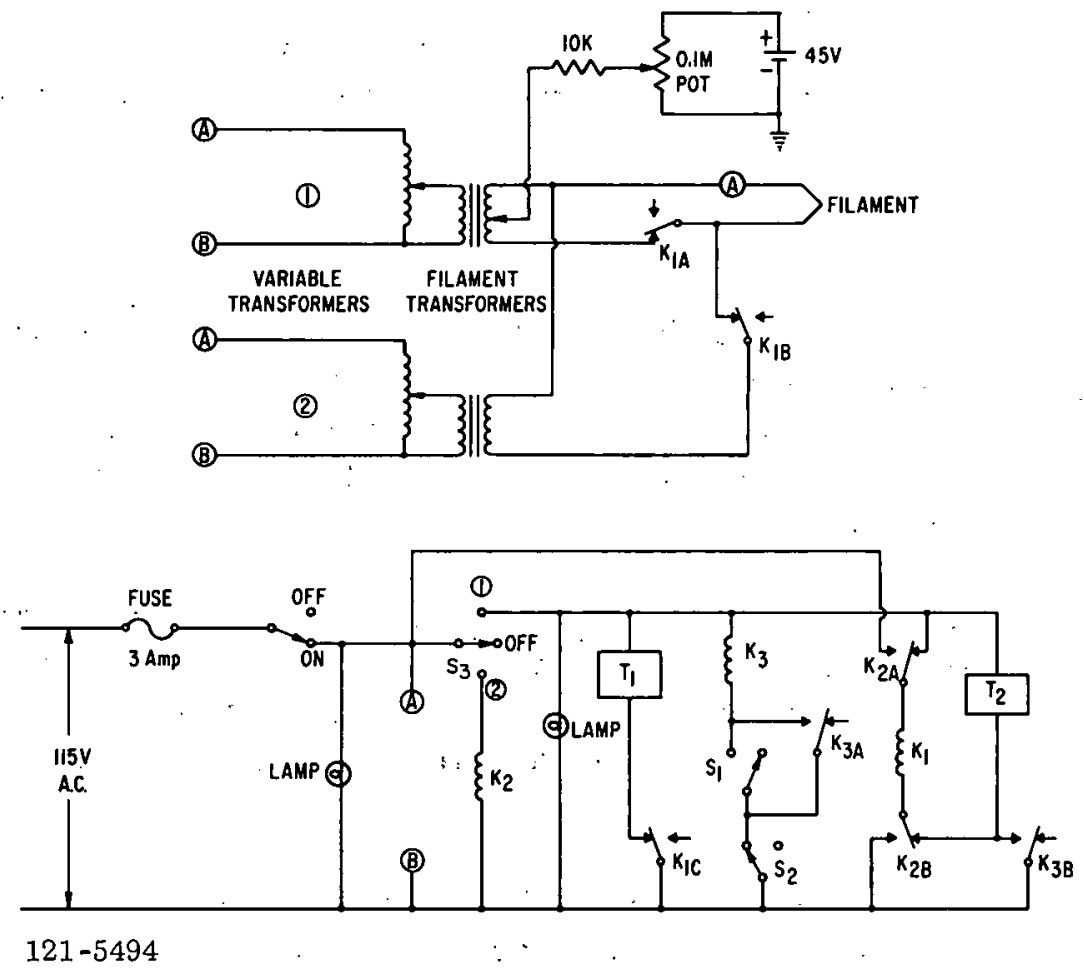

Fig. 15. Circuitry for Flashing Filament

When switch $\mathrm{S}_{3}$ is in the OFF position, the timing circuitry is isolated, but the filament supply can be regulated by variable transformer 1 . When switch $S_{3}$ is in position'2, relay $K_{2}$ actuates relay $K_{1}$ and the filament current supply is now regulated by variable transformer 2 .

The variable transformers (Staco Powerstats, Type V) supply 0.$120 \mathrm{~V}$ ac to the filament transformer (Knight, Type 6-K-34-VBR). The filament transformers supply up to $6.3 \mathrm{~V}, 10 \mathrm{~A}$, to the filaments. A bias of 0 to $\pm 45 \mathrm{~V}$ dc with respect to ground can be applied to the filament through the center tap at the output of the filament transformers. The time-delay timers 
(Industrial Timer Corp. Type CSF-5M) have a range of 0-5 min delay. Relay $K_{2}$ (Potter and Brumfield Type $M R-11 \mathrm{~A}$ ) is a double-pole, doublethrow, medium-duty power relay. $K_{1}$ and $K_{3}$ are Potter and Brumfield general-purpose relays $\left(K_{1}: 3 P D T\right.$ Type KRPl4AN; $K_{3}$ : DPDT Type KRPl IAN) with a maximum time of switching of $15 \mathrm{msec}$ when actuated.

\section{B. Experimental Prelude}

The behavior and interpretation of the surface-ionization properties of rhenium-salt systems depend in large part on an adequate experimental approach. Reasons for the choice of rhenium as the surfaceionization substrate are given here. The preparation and treatment of the rhenium filaments prior to their use as surface-ionization sources is described. The metal surface is further characterized by definitive experimental studies on its adsorptive properties and work-function values. The preparation and choice of lithium compounds are described with respect to chemical purity.

\section{Materials Section}

The physical and chemical properties ${ }^{161}$ of rhenium that make rhenium an ideal surface-ionization emitter are discussed here. The bakeout procedure for preparing atømically clean rhenium filaments is described. The lithium salts used are characterized by the level of impurities present in the salt.

a. Characteristics of Rhenium. Of the refractory metals, rhenium has the second highest melting point, $3180^{\circ} \mathrm{C}$, and next to platinum, has the highest work function. Rhenium has a hexagonal-close-packed crystal lattice with the following parameters: ${ }^{147} a_{0}=2.760 \pm 0.001 \AA$, $c_{0}=4.458 \pm 0.001 \AA$.

Nearly all recrystallized metals with a body-centeredcubic lattice, like tungsten and molybdenum, suffer an abrupt transition from a relatively ductile state at high temperatures to a brittle state at lower temperatures. The transition takes place within a narrow temperature interval. Rhenium metal possesses considerable ductility at all temperatures. ${ }^{147}$ Rhenium wires can be subjected to prolonged heating above the recrystallization temperatures without subsequent mechanical failure when cooled to low temperatures.

The electrical specific resistance of rhenium, which is about four times that of tungsten, is useful for maintaining the power dissipated by the emitter and for keeping the supply voltage constant.

Rhenium is characterized by its relatively low chemical activity, compared to tungsten, tantalum, or molybdenum. Among the 
chemical properties of rhenium, the most,interesting are its reactions with oxygen, nitrogen, water vapors, and carbon-containing compounds, which are usually present in the composition of residual gases in mass-spectrometer sources. Rhenium does not form carbides, but carbon diffuses into rhenium at high temperatures, forming a solid solution. Rhenium filaments can be used successfully in carbon atmospheres at high temperatures. ${ }^{162}$

Rhenium does not form nitrides when heated in nitrogencontaining atmospheres as does tungsten. ${ }^{162,163}$ In oxygen, rhenium reacts weakly compared to tungsten. ${ }^{8,164}$ At $1450^{\circ} \mathrm{K}$, the emis sion properties of rhenium deteriorate at an oxygen pressure of $10^{-6}$ Torr, but this occurs for tungsten at an oxygen pressure of $10^{-8}$ Torr.

Weiershausen ${ }^{165}$ studied the dependence of the work function of polycrystalline rhenium on the surface concentration of oxygen. He found that the surface work function increased a maximum of $1.75 \mathrm{eV}$ at near-monolayer coverage.

Rhenium is considerably more resistant than tungsten to reactions of water vapor. Reactions of the water cycle lead to erosion of metallic emitters and thus impair the stability of their operation and shorten their lives. At a water-vapor pressure of about $10^{-4}$ Tor $r$ and a temperature near $2000^{\circ} \mathrm{K}$, rhenium erodes 20 times slower than tungsten. ${ }^{162}$.

Several gaseous rhenium oxides are volatilized from rhenium surfaces on which nitrates have been deposited. ${ }^{166}$ Volatilization occurs below $500^{\circ} \mathrm{C}$.

The suitability of rhenium as a surface-ionization emitter of optimum properties having been established, the choice of the alkali metal salts was considered. For systems with low ionization potentials and high work functions, the efficiency of surface ionization is so great as to swamp out all other effects. For low work function and high ionizationpotential systems, the efficiency is so low that successful detection and analysis become difficult. This is illustrated in Fig. 16 where the fraction ionized, $\beta$, or the ratio of ions produced to atoms reaching the surface, given by

$$
\beta=\frac{Z_{+}}{Z_{+}+Z_{0}}=\frac{\alpha}{l+\alpha}=\left(1+\frac{g_{0}}{g_{+}} \exp \frac{I-\phi}{k T}\right)^{-1} .
$$

is plotted against temperature as a function of the parameter $\Delta=I-\phi$ in electron volts for alkali-metal atoms for which $g_{0} / g_{+}=2$. The difficulty in the surface ionization of lithium ( $I=5.39 \mathrm{eV})$ on tungsten $(\phi=4.52 \mathrm{eV})$, for which $\Delta$ is highly positive, is apparent from Fig. 16. Indeed, any contamination of the surface that alters the effective work function by a fraction of an electron, volt will greatly affect the fraction ionized. 


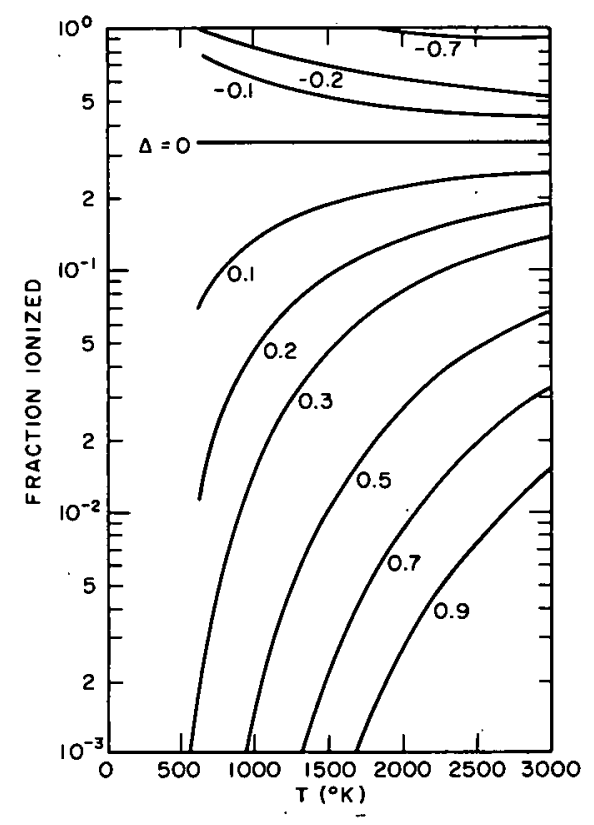

$121-5502$

Fig. 16. Fraction Ionized vs Temperature for the Alkali Metals as a Function of the Parameter $\Delta=\mathrm{I}-\phi$
For polycrystalline rhenium, the effective surface-ionization work function is but slightly less than the ionization potential. of lithium. ${ }^{161}$ Therefore, the systems of lithium and lithium salts on polycrystalline rhenium appear as a possible successful compromise in this study.

b. Preparation of Rhenium Filaments. Rhenium of high purity (99.99\%) was cut in the form of a ribbon $0.05 \mathrm{~cm}$ wide and $2 \mathrm{~cm}$ long from 1-mil-thick sheets obtained from Chase Brass and Copper Company, Waterbury, Connecticut. The principal impurities given by the manufacturer (in parts per million) are tungsten (15), carbon (5.0), molybdenum (4.0), iron (3.0), potassium (1.0), oxygen (0.5), silica (0.5), hydrogen (0.2), nitrogen (0.2), and sodium (0.1). Lesser amounts of other metals are present. The lithium impurity is not given.

The ribbon was first cleaned by boiling successively in carbon tetrachloride, acetone, and ethyl alcohol. The ribbon then was outgassed in a Pyrex
vacuum system by slowly raising the temperature to $2700^{\circ} \mathrm{K}$ while the total gas pressure was kept below $5 \times 10^{-6}$ Torr. Prebaking was continued for $20 \mathrm{~min}$, principally to remove the major portions of the occluded alkali metals in the rhenium filament. Several filaments were prepared in this manner.

After being spotwelded onto 60-mil tungsten rods previously connected to Kovar feedthroughs, the rhenium ribbon was further outgassed for several hours at greater than $2400^{\circ} \mathrm{K}$ in the ion source of the Bendix time-of-flight mass spectrometer. Filament baking continued overnight at $2000^{\circ} \mathrm{K}$. This heating also served to bake out the ion-source region. Alternating current was used for heating, since a direct current produces a roughened surface. ${ }^{167,168}$

c: Sample Preparation. Reagent-grade lithium chloride, lithium bromide, lithium nitrate, and lithium sulfate were separately weighed and dissolved in distilled water to give stock solutions of $\mathrm{l} g$ of salt per $100 \mathrm{ml}$ of solution. The concentration of the halide salts is approximate, due to their hydroscopic nature.

Lithium iodide was prepared by reacting a weighed amount of lithium carbonate with a $5 \%$ excess of hydriodic acid. The solution was evaporated to dryness and warmed to remove the excess hydriodic acid. 
The $1.5 \%$ hypophosphorus acid used as a preservative in hydriodic acid solutions was decomposed on heating. The lithium iodide was dissolved in distilled water to give a concentration of $1 \mathrm{~g}$ of salt per $100 \mathrm{ml}$ of solution.

The chemicals were obtained from J. T. Baker Chemical Company, Phillipsburg, New Jersey, and used without further purification. The principal impurities present in the halides (in weight percent) were insolubles (0.01), sulfate $(0.01)$, calcium (0.015), and barium (0.003). In addition, potassium (0.1) and sodium (0.2) were present in the lithium carbonate used to make the lithium iodide. Other impurities were present at less than $0.001 \%$.

The lithium sulfate and lithium nitrate contained insolubles (0.005), sodium (0.01), and other impurities (0.005). The level and nature of the impurities present in the lithium salts were not sufficient to affect the surface-ionization studies reported in Chapter V.

An aliquot of a particular solution was transferred to the sample filament using a micropipette and evaporated in air by slight electrical heating of the filament. The filament was then placed in the ion source as described in Section IV.A.2. In most cases, the sample size was of the order of $1 \mathrm{mg}$ of salt.

Lithium metal of greater than $99 \mathrm{a} / 0{ }^{7} \mathrm{Li}$ was melted directly onto a clean rhenium ribbon in an argon atmosphere and served as a source for atomic lithium.

\section{Preliminary Procedures}

In this section, the rhenium metal surface is characterized by definitive experimental studies on adsorptive properties and workfunction measurements. The results are discussed with respect to the experimental values of sticking coefficients and work functions obtained by other workers.

a. Flash Desorption of Residual Gases. The number of adsorbed residual gas atoms on the rhenium surface-ionization filament for various coeling times was calculated conveniently by the flash-filament method. The filament was heated rapidly, and the pressure increase in a well-defined volume caused by the desorbed gas was measured. If the filament of area A reaches its desorption temperature in a time short compared to the time required by the system to pump away the desorbed gas, the number of desorbed. molecules per square centimeter is obtained from Eq. 3.104 as

$$
\sigma_{\mathrm{t}}=\frac{\mathrm{V}}{\mathrm{AkT}}(\mathrm{p}-\mathrm{peq})=\sigma_{0} \theta
$$


where $\sigma_{t}$ is the surface concentration after a cooling time $t$, and the other symbols were defined previously.

The rate at which the coverage increases with the length of the adsorption period yields the sticking probability s, since, from Eq. 3.101 ,

$$
s=\frac{(2 \pi \mathrm{mk} T)^{1 / 2}}{\because P_{e q}} \frac{d \sigma_{t}}{d t}=\frac{1}{Z} \frac{d \sigma_{t}}{d t}
$$

where $Z$ is the flux of molecules in the gas phase striking the filament in molecules $\mathrm{cm}^{-2} \mathrm{sec}^{-1}$. Using Eq. 3.104 in Eq. 3.101, we obtain

$$
s=\frac{V}{A k T Z} \frac{d \Delta p}{d t}
$$

If $\Delta p$ and $p$ are measured as a function of the adsorption interval $t$, the sticking probability can be calculated as a function of the coverage for a particular adsorption temperature of the ribbon. In the following experiments, the ion current of a particular species was taken to be proportional to its pressure.

The principal mass peak of the residual gases in the mass spectrometer is at 28 atomic mass units and consists mainly of molecular nitrogen together with some carbon monoxide. If the effect of carbon monoxide is ignored for the moment, the pressure of the molecular nitrogen is related to the molecular ion current I by

$$
\mathrm{p} .=\mathrm{KTI} \text {, }
$$

with $K$, the mass-spectrometer sensitivity constant, given by

$$
K=\frac{k}{E Q I(e) \ell \gamma} \text { dynes } \mathrm{cm}^{-2} \mathrm{~A}^{-1}{ }^{\circ} \mathrm{K}^{-1} \text {, }
$$

where $\mathrm{k}$ is Boltzmann's constant, $\mathrm{E}$ is the efficiency of ion collection, $Q$ is the absolute cross section for ionization in square centimeters, I(e) is the electron-beam current in amperes, $l$ is the active path length of electrons in centimeters, and $\gamma$ is the gain of the electron multiplier.

tained from

The coverage due to species of mass 28 can then be ob-

$$
\sigma_{t}=\frac{\mathrm{V}}{\mathrm{Ak}} \mathrm{K} \Delta \mathrm{I}
$$


and the sticking coefficient from

$$
s=\frac{V}{A k T} \frac{(2 \pi m k T)^{1 / 2}}{I_{e q}} \frac{d \Delta I}{d t}=\frac{V K d \Delta I}{A k Z d t}
$$

using Eq. 4.27 in Eqs. 3.104 and 3:102.

In the preliminary experiments, the rhenium filament was situated between the backing plate and the first ion-accelerating grid of the mass-spectrometer ion source. These grids were $1 / 4$ in. apart. When the filament was flashed, the molecular-nitrogen ion current was approximately two orders of magnitude greater than could be accounted for, based on filament size. This was due in large measure to desorption of gas from the accelerating electrodes.

The rhenium filament was therefore placed in the 4 -in.-diam cylindrical cavity above the ion source proper, $8 \mathrm{~cm}$ from the electronbeam path. This minimized gas desorption from the surrounding walls on flashing.

The mass-spectrometer constant, K, was determined as follows: Rhenium was used as the standard for calibration purposes. The recent definitive determination of the vapor pressure of rhenium by Plante and Szwarc ${ }^{169}$ relates the vapor pressure to the temperature by the equation

$$
4.5756 \log \mathrm{p}(\mathrm{atm})=31.86-\frac{180200}{\mathrm{~T}}
$$

in the range of $2300-3050^{\circ} \mathrm{K}$.

From the measured temperature of the rhenium filament, the vapor pressure of rhenium and the consequent flux of rhenium particles leaving the filament were calculated. The fraction of the flux intercepted by the electron beam was determined from the known relative geometry and areas of the filament and the electron beam using the cosine distribution law. The geometry factor, $G$, was calculated to be $2.41 \times 10^{-4}$. From the pressure of rhenium atoms in the electron beam and the measured ion current of the rhenium isotope ions ${ }^{185} \mathrm{Re}(37.07 \%)$ and ${ }^{187} \mathrm{Re}$ $(62.93 \%)$, the spectrometer constant was calculated by Eq. 4.27. Thus the effective pressure of rhenium, $\mathrm{p}_{e}$, in the electron-beam volume is

$$
\mathrm{p}_{\mathrm{e}}=\frac{2 \mathrm{G}}{3 \pi} \mathrm{p}_{\mathrm{f}}=\mathrm{KTI}
$$


where $p_{f}$ is the pressure at the filament determined from the measured filament temperature. With the filament at $2747 \pm 17^{\circ} \mathrm{K}$ and a total measured ion current of $7.5 \pm 0.1 \times 10^{-10} \mathrm{~A}$, the spectrometer constant is

$$
\mathrm{K}=1.06 \pm 0.50 \text { dynes } \mathrm{cm}^{-2} \mathrm{~A}^{-1}{ }^{\circ} \mathrm{K}^{-1} \text {. }
$$

The large standard error in the spectrometer constant arises from the error in the determination of the pressure. The errors inherent in the enthalpy and temperature determinations give rise to a $25 \%$ error in the pressure.

The ion current due to mass 28 was measured with the rhenium surface-ionization filament at room temperature. The pressure of mass 28 in the electron-impact ionization beam (and in the total volume of the mass spectrometer) is related to the ion current by

$$
\mathrm{p}_{28}=\mathrm{K}_{28} \mathrm{~T}_{28} \mathrm{I}_{28}
$$

The spectrometer constant for mass 28 is related to that for rhenium by the expression

$$
K_{28}=\frac{K_{R e} Q_{R e} \dot{\gamma}_{R e}}{Q_{28} \gamma_{28}} .
$$

The relative ionization cross sections were obtained from $\mathrm{Mann}^{170}$ and Harrison et al. ${ }^{171}$ with $Q_{\mathrm{Re}}=7.25 \times 10^{-16} \mathrm{~cm}^{2}, Q_{\mathrm{N}_{2}}=2.81 \times 10^{-16} \mathrm{~cm}^{2}$, and $Q_{C O}=3.04 \times 10^{-16} \mathrm{~cm}^{2}$. The ionization cross sections for molecular nitrogen and carbon monoxide are not too different, and since most of the species contributing to the mass 28 peak are molecular nitrogen, the cross section for molecular-nitrogen ionization was used. Inghram and Hayden ${ }^{5}$ discussed the number of secondary electrons emitted per incident ion at the electron multiplier detector for various ion masses and energies. A rough approximation for the $\gamma_{\mathrm{Re}} / \gamma_{28}$ ratio may be obtained from the square root of the mass dependence. ${ }^{5,172}$ At an ion-acceleration potential of $3000 \mathrm{~V}, \gamma_{\mathrm{Re}} / \gamma_{28} \cong 0.4$. The spectrometer constant for molecular nitrogen is then $1.09 \pm 0.52$ dynes $\mathrm{cm}^{-2} \mathrm{Am}^{-1}{ }^{\circ} \mathrm{K}^{-1}$. For a measured molecular ion current of $2.1 \times 10^{-8} \mathrm{~A}$ for mass 28, the partial pressure for molecular nitrogen is calculated to be $6.9 \pm 3.5 \times 10^{-6}$ dynes $/ \mathrm{cm}^{2}$.

The spectrometer constant for molecular nitrogen is checked by bleeding in nitrogen gas until a steady-state flow is attained. The Bayard-Alpert hot-cathode ionization gauge gave a reading of $1.7 \mathrm{x}$ $10^{-6}$ Torr. With the electron-multiplier gain reduced by a factor of 100 to avoid multiplier saturation, a mass 28 current of $7.8 \times 10^{-8} \mathrm{~A}$ was recorded. These readings correspond to an approximate spectrometer constant of $\mathrm{K}=0.97$ dynes $\mathrm{cm}^{-2} \mathrm{~A}^{-1}{ }^{\circ} \mathrm{K}^{-1}$. 
The spectrometer constant for rhenium was determined for a steady-state, one-way flux of rhenium atoms through the electron ionizing beam and then used to determine the mass 28 spectrometer constant for an isotropic flux of mass 28 particles. However, for highaccelerating potentials, there is no discrimination due to velocity components perpendicular to the acceleration potential of the spectrometer. The measured ion currents correspond to the effective number density of particles within the electron-beam volume. Once ionized, the ions are held in the potential well of the electron beam until pulsed out of the ion source.

The two most important variables that are incorporated in the spectrometer constant in Eq. 4.28 are the electron multiplier gain, $\gamma$, and the electron-beam current, $I(e)$. The latter is measured by an electron trap meter and can be monitored. The multiplier gain can be adjusted, within limits.

The ribbon was heated to $2400^{\circ} \mathrm{K}$ for $30 \mathrm{~min}$ to remove any adsorbed gases. The filament was cooled, and after a certain adsorption interval, the filament was suddenly flashed to $2150^{\circ} \mathrm{K}$. The change in the mass 28 current was measured by the analog system of the mass spectrometer. The analog voltage output was observed as a trace on the oscilloscope screen or recorded on a Honeywell Visacorder Model $906 \mathrm{C}$ recorder with a frequency response of $200 \mathrm{~Hz}$. The multiplier gain was reduced by a measured factor, when required to avoid saturation of the electron multiplier.

The flashing and cooling intervals were taken either from the Varian Type G-llA potentiometric recorder traces of the pressure pulses detected by a hot-cathode ion gauge (Veeco, RG-75) or directly from the Honeywell recorder tracings of the mass 28 current.

When the flash occurs, the concentration of adsorbed particles at the rhenium surface changes, changing the partial pressure of the gas. At high rates of adsorbent heating, the rate of change of the surface concentration is small on the falling-off portion of the desorption curve. This affords another means of determining the characteristic pumping time of the system walls and diffusion pump. The pumping action by the filament is so small with respect to the system pumping that it is not detected by observations on this portion of the trace. This is borne out by observing that, within experimental error, the decay time after flashing and during the flash is the same.

It is desirable to flash the filament more than once at the end of a cold interval and the beginning of another. A single short flash may allow readsorption of part of the desorbed gas. Too long a series of flashes, or a single long flash, results in a rise in ambient temperature, 
causing slight degassing of the surrounding metal parts. Flashing to $2150^{\circ} \mathrm{K}$ removed all nitrogen from the filament, as evidenced by fact that increasing the temperature above this value produced no increase in ion current. The flash duration in these experiments was approximately $3 \mathrm{sec}$.

The variation in resistance or temperature with respect to the time of heating or cooling can be observed conveniently on an oscilloscope screen adapting a circuitry presented by Hagstrum. ${ }^{30}$ The vertical deflection was made proportional to the bridge unbalance (i.e., resistance) using the ac bridge circuitry previously described in Section IV.A.3.a(2). A typical display is shown in Fig. 17. The maximum temperature $\left(2150^{\circ} \mathrm{K}\right)$ was reached in less than $0.4 \mathrm{sec}$, with an initial rapid exponential rise. Figure 18 is a typical oscilloscope trace of the mass 28 ion-current pulse during time of flashing with the filament between the ion-accelerating electrodes. During desorption the heating rate was fast enough to coalesce the peaks arising from different states or binding energies of nitrogen on rhenium as well as from the walls. The characteristic pumping time of the system may be obtained from Fig. 19. Air was allowed to flow into the system at a constant rate. A new steady-state pressure, and hence mass 28 ion current, was attained. When the flow was stopped, the mass 28 ion current was decreased to the base steady-state current. The characteristic pumping time obtained from Eq. 3.95 or from the initial slope of the currenttime curve was $\tau=0.6 \mathrm{sec}$. The ion current dropped to within $1 \%$ of the steady-state value for no flow in about $7 \mathrm{sec}$.

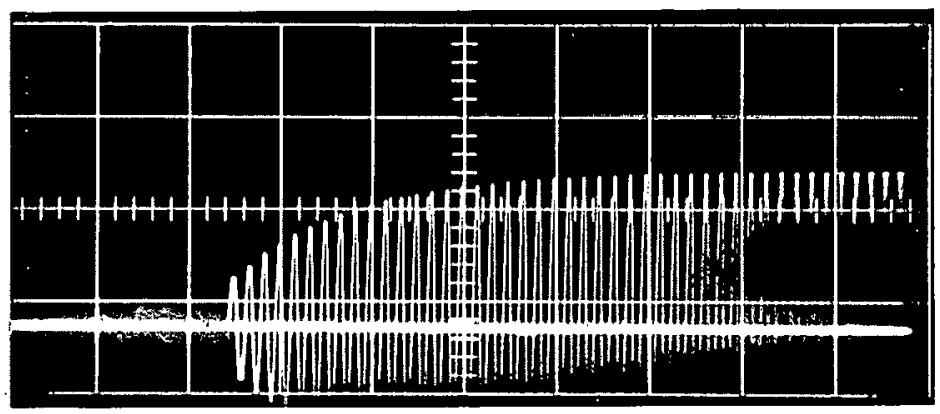

Fig. 17

Variation in Bridge Unbalance during Flashing. Horizontal deflection: $0.1 \mathrm{sec} / \mathrm{cm}$; vertical deflection: $0.5 \mathrm{~V} / \mathrm{cm}$

Fig. 18

Mass 28 Voltage Pulse during Flashing. Horizontal deflection: $50 \mathrm{msec} / \mathrm{cm}$; vertical deflection: $5 \mathrm{~V} / \mathrm{cm}$.

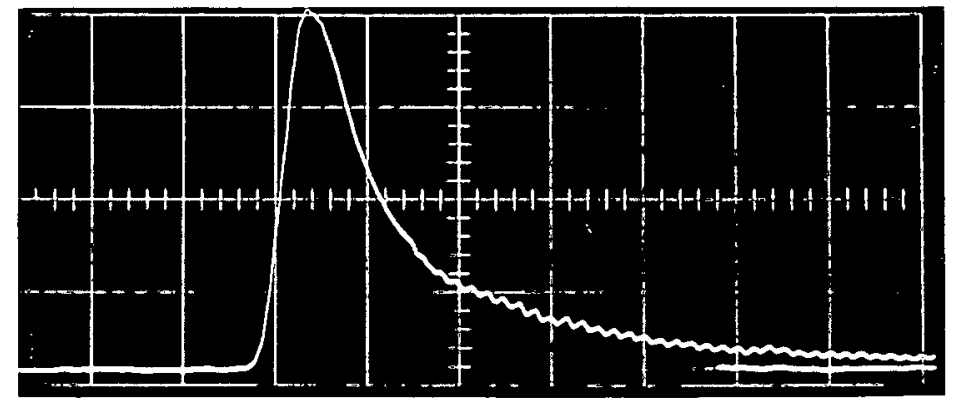




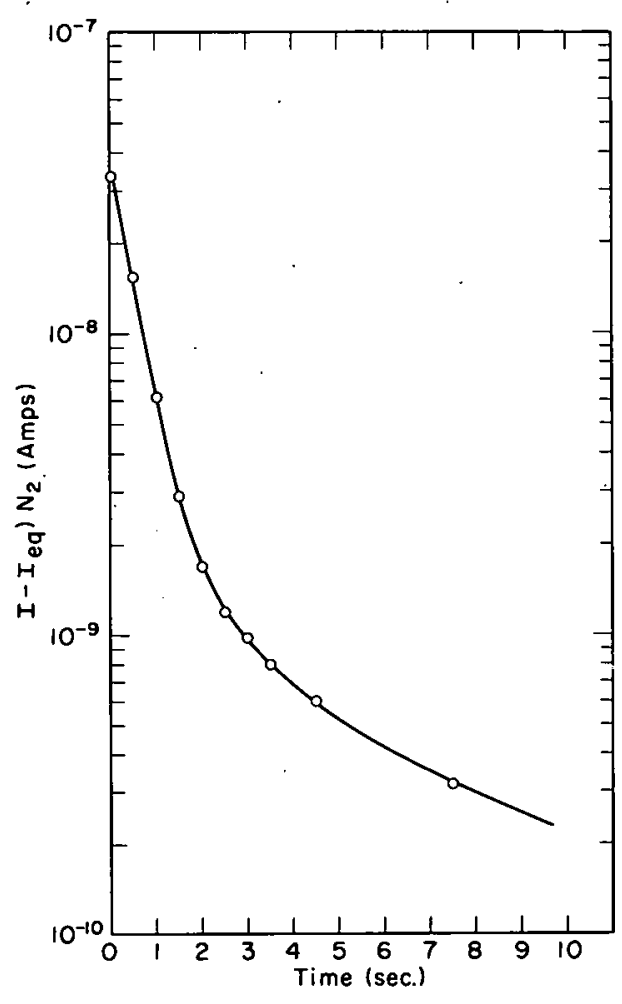

$121-5517$

Fig. 19. Drop in $\mathrm{N}_{2}^{+}$Ion Current vs Time after Flow Stoppage
These results illustrate that the desorption temperature of the filament is reached in a time short with respect to the time required by the system to pump away the desorbed gas. The simplified expression, Eq. 4.29, can therefore be used.

The desorption measurements were carried out as follows: The total pressure in the mass spectrometer was $8.6 \times 10^{-9}$ Torr, as measured by the Bayard-Alpert gauge. A constant flow of molecular nitrogen was valved into the mass spectrometer. The steady-state mass 28 ion current measured was $7 \times 10^{-9} \mathrm{~A}$, with a reduction of 100 in the multiplier gain. This corresponds to a molecular nitrogen pressure of $2.3 \mathrm{x}$ $10^{-4}$ dynes $/ \mathrm{cm}^{2}\left(1.7 \times 10^{-7}\right.$ Torr $)$. The hotcathode ion gauge read $1.6 \times 10^{-7}$ Torr.

Figure 20 shows the variation of the ion-current pulse height with cooling time. Each point represents an average of three current readings for a given cold interval. For cooling intervals of $15 \mathrm{sec}$

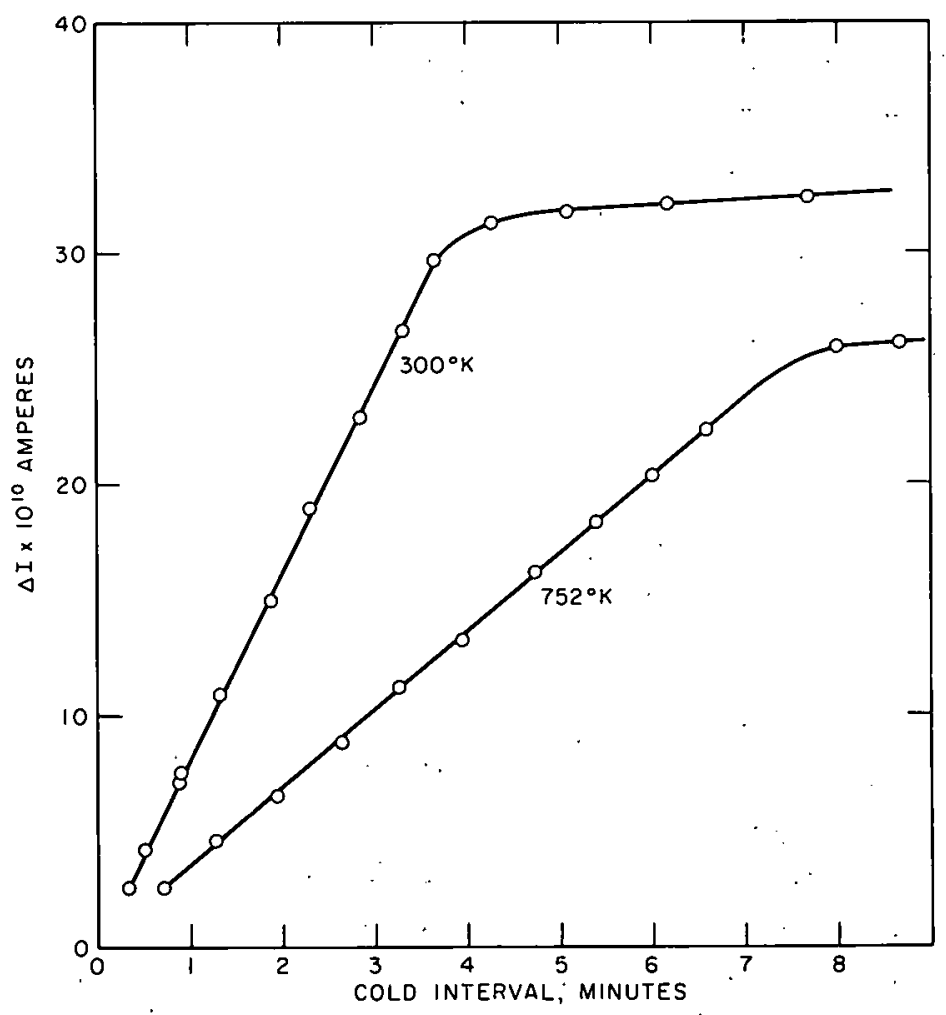

121-5501

Fig. 20. Mass 28 Ion-current Pulse Height vs Cooling Time 
or less, the error is as much as $100 \%$ due to the small difference, $\Delta I$, between relatively large numbers. For cooling intervals greater than $1 \mathrm{~min}$, the deviation from the average $\Delta \mathrm{I}$ is of the order of $\pm 3 \%$ 。

The nitrogen desorption curve for the filament at $752^{\circ} \mathrm{K}$ during the cold interval was obtained by a different method. After an adsorption interval, the gas flow was stopped. Within $10^{-}$sec, the mass 28 ion current had decreased to a pointwhere, on flashing, a sufficient deflection on the recorder could be measured. The cycle was repeated by resumption of the gas flow. The steady-state gas flow was maintained at a mass 28 ion current of $2.3 \pm 0.2 \times 10^{-8} \mathrm{~A}$, corresponding to a pressure of $7.52 \pm 0.65 \times 10^{-4}$ dynes $/ \mathrm{cm}^{2}$. After the filament was cleaned, another adsorption interval was recorded, the gas flow stopped, and flash desorption initiated.

The ion-current pulse height versus cooling time for the filament at $752^{\circ} \mathrm{K}$ is shown in Fig. 20. The change in slope is typically considered to be the point where monolayer coverage occurs.

During the interval between gas-flow stoppage and flashing, adsorption of nitrogen onto the filament continued, but at a reduced rate. This added adsorption was considered negligible at adsorption intervals of greater than 1 min. However, the initial sticking coefficient calculated was slightly low. The sticking coefficient was calculated to be 0.008 and 0.001 with the adsorbing filament at room temperature and $752^{\circ} \mathrm{K}$, respectively. The estimated error is a factor of two.

It has been shown ${ }^{173}$ that anomalous results may be obtained from flash-filament experiments if no account is taken of the possibility that other residual gases play a part in the observed adsorptiondesorption phenomena. The relatively low residual-gas pressure and insignificant variations in the ion currents from other gases during flash desorption suggest no anomalies.

At various times during the experiment, the mass 14 ion was monitored. This ion arises from fragmentation of the nitrogen molecule during electron bombardment. The ratio of the mass 28 to mass 14 ion current was essentially. constant; showing that the phenomenon observed is characteristic of molecular nitrogen.

Table $X$ gives the sticking coefficients and the saturation surface coverage for various gases on clean polycrystalline rhenium wires: The sticking coefficient for nitrogen on rhenium is less than that on tungsten by more than an order of magnitude at comparable conditions of temperature and pressure. The sticking coefficients decrease markedly. at high surface concentrations and increasing temperature of the substrate. For nitrogen on rhenium, the sticking coefficient is less than 0.0001 at the 
saturation surface coverage. The quantitative differences in the values for nitrogen on rhenium listed in Table $X$ can be accounted for by the variations in the polycrystalline wires used.

TABLE X. Sticking Probability and Saturation Surface Coverage of Gases on Polycrystalline Rhenium Filaments

\begin{tabular}{lcccc}
\hline Gas & Sticking Coefficient & $\begin{array}{c}\text { Saturation Surface } \\
\text { Coverage, } \\
\text { molecules } / \mathrm{cm}^{2}\end{array}$ & $\begin{array}{c}\text { Atom Ratio } \\
\text { Gas } / \text { Rhenium }\end{array}$ & Reference \\
\hline $\mathrm{N}_{2}$ & $0.008\left(300^{\circ} \mathrm{K}\right)$ & $1.2 \times 10^{14}$ & $1 / 6$ & This work \\
$\mathrm{N}_{2}$ & $0.001\left(752^{\circ} \mathrm{K}\right)$ & $0.98 \times 10^{14}$ & $1 / 10$ & 118 \\
$\mathrm{~N}_{2}$ & $0.009\left(300^{\circ} \mathrm{K}\right)$ & $7 \times 10^{13}$ & $1 / 5$ & 174,175 \\
& $0.0024\left(291^{\circ} \mathrm{K}\right)$ & $1.5 \times 10^{14}$ & & \\
$\mathrm{CO}$ & $0.001\left(720^{\circ} \mathrm{K}\right)$ & $1.0 \times 10^{14}$ & $3 / 4$ & 176,175 \\
$\mathrm{H}_{2}$ & $0.0006\left(870^{\circ} \mathrm{K}\right)$ & $0.4 \times 10^{14}$ & & 177 \\
\hline
\end{tabular}

Utsugi and Gomer ${ }^{178}$ determined the number of surface atoms present per square centimeter for the hexagonal-close-packed structure of rhenium to be $6.6 \times 10^{14}$. Taylor ${ }^{179}$ gave a value of $6.9 \mathrm{x}$ $10^{14}$ atoms $/ \mathrm{cm}^{2}$. The atom ratios in Table $\mathrm{X}$ were obtained using a rhenium surface atom concentration of $7 \times 10^{14}$ atoms $/ \mathrm{cm}^{2}$.

The constancy of the sticking coefficient during the initial stages of adsorption and its dependence on temperature can be qualitatively accounted for in terms of a precursor adsorption state, perhaps consisting of physically adsorbed molecules. ${ }^{118}$ These may either desorb or pass over to the final chemisorbed state, and the decrease in sticking probability with increasing temperature arises because desorption becomes more probable at higher temperatures. The initial constancy of the sticking coefficient can be explained by the fact that molecules may migrate over the surface to vacant sites.

As with tungsten, there are several binding states for the various gases with rhenium. A strongly bound $\beta$ state desorbs above $1000^{\circ} \mathrm{K}$. This high-temperature $\beta$ state is about $8 \%$ populated before the lower binding $\alpha$ state begins to fill. ${ }^{118,174}$ The $\alpha$ state desorbs below about $500^{\circ} \mathrm{K}$. For hydrogen on rhenium, both binding states are desorbed below $600^{\circ} \mathrm{K}$. The assignment of different binding states to different crystal faces has not been confirmed experimentally.

It has been suggested ${ }^{174}$ that the desorption kinetics of the $\beta$ state from rhenium seems to have an order greater than unity. This is 
based on the observation that the time for the desorption maximum to be reached decreased monatomically by $15 \%$ when the coverage altered from 6 to $60 \%$. Such a trend was not evident in the experiments reported here. If adsorption is molecular to the precursor state, with subsequent activated dissociative chemisorption of nitrogen atoms leading to molecular evaporation by association on heating, the process would obey second-order kinetics. Further details are contained in a review by Ehrlich ${ }^{4}$ and the references in Table $\mathrm{X}$.

Flash desorption of oxygen on metals has generally proved unsuccessful for obtaining precise surface coverage and sticking-coefficient data. At high coverage, the desorbed species contain appreciable metal oxides. In addition, carbon monoxide is evolved, due to the presence of carbon impurities. Ion masses corresponding to rhenium, $\mathrm{ReO}_{2}$, and $\mathrm{ReO}_{3}$ were observed on flashing the rhenium filament to $2700^{\circ} \mathrm{K}$ after a cooling period of about $30 \mathrm{~min}$ in a vacuum of $5 \times 10^{-9}$ Torr. Repeated flashings at short intervals evolved only rhenium.

Trapne $11^{40}$ briefly summarized the adsorption character istics of oxygen on metals. Chemisorption of oxygen is rapid on all metals except gold, implying a zero or low-activation energy for adsorption. Oxygen is adsorbed dissociatively and desorbs as atoms. On tungsten, oxygen evaporates as atoms at about $1600^{\circ} \mathrm{K}$. Slow oxygen uptake after fast chemisorption is due to the start of bulk oxidation, resulting in the breaking of metal-metal bonds. The metal surface is replenished, and the process can occur in vacuum at room temperature.

Oxygen chemisorption causes a surface dipole to form which is negative outwards, and this increases the work function. A mechanism such as metal surface replenishment would tend to offset the increase in work function. This was the case with the iron-oxygen system. ${ }^{180}$ However, the ionic character of surface bonds for oxygen on tungsten is of the order of $50 \%$ while for hydrogen, nitrogen, and carbon monoxide the ionic character of the surface bond is less than $10 \%$. It is therefore expected that oxygen adsorption will have a greater effect than other residual gases on the work function For oxygen-rhenium systems, the work-function change at a given ternperature is not expected to be as pronounced as with tungsten.

b. Thermionic Work Function. The rhenium ribbons used have a polycrystalline surface structure. Electron emission and surface ionization of atoms on metals with a polycrystalline surface structure have a number of distinctive features. ${ }^{14,20,80}$ Field-emission patterns from rhenium points show that various faces of rhenium single crystals have different work functions. ${ }^{164}$ The principal contribution to the thermionic current is made by faces with a low work function. The effective thermionic work function of a polycrystalline surface, $\phi_{\mathrm{e}^{*}}^{*}$ approaches the minimum local values of the surface work function, $\phi_{\min }$. 
The quantity $\phi_{e}^{*}$ may be somewhat different for various structures of the same material. However, measurements of thermionic electron emission, made under conditions in which the current is collected from an area considerably greater than the size of individual faces on the surface, indicate that standard heat treatment results in similar surface structure. This explains the nearly identical values of the thermionic constants for emitters of similar shapes reported in different works.

The experimental determination of the thermionic work function of the polycrystalline rhenium ribbon to be used in the surface ionization studies follows. The filament ribbon was prepared as previously described.

For polycrystalline emitters consisting of a collection of small areas $S_{i}$ with work functions $\phi_{i}$, the electron emission current is given by

$$
I=\sum A T^{2} S_{i} \exp \left(-\frac{\phi_{i}}{k T}\right)
$$

where $A$ is the thermal-emission constant in $\mathrm{A} \mathrm{cm}^{-2}{ }^{\circ} \mathrm{K}^{-2}$, and $\mathrm{T}$ is the surface temperature of the emitter. If the Richardson plot is linear,

$$
I=A^{*} T^{2} S \exp \left(-\frac{\phi_{e}^{*}}{k T}\right)
$$

where $A^{*}$ and $\phi_{\mathrm{e}}^{*}$ are the effective emission constants for the polycrystalline surface, and $S$ is the total area of the emitting surface.

The experimental arrangement within the ion source of the mass spectrometer is shown schematically in Fig. 21. Alternating current was. used to heat the filament. Its temperature was measured by optical pyrometry. A measured dc bias with respect to the grounded platinum shield was applied by means of a $300-\mathrm{V}$ battery. The total emitted current was measured by recording the potential drop across a suitable resistor. The variation in the total electron current emitted at a given filament temperature with respect to the filament voltage established the saturation region of emission.

Some of the electrons were accelerated through a slit in the platinum shield and impinge on a collector ribbon of polycrystalline rhenium of similar shape and size as the emitting ribbon. The current from the impinging electrons was measured with an Applied Physics Corporation Model 30 vibrating-reed electrometer and recorded. A positive bias was applied to the collector ribbon to avoid reflection and secondary emission. The mass-spectrometer collimating magnets at the ion source were removed during this experiment. 


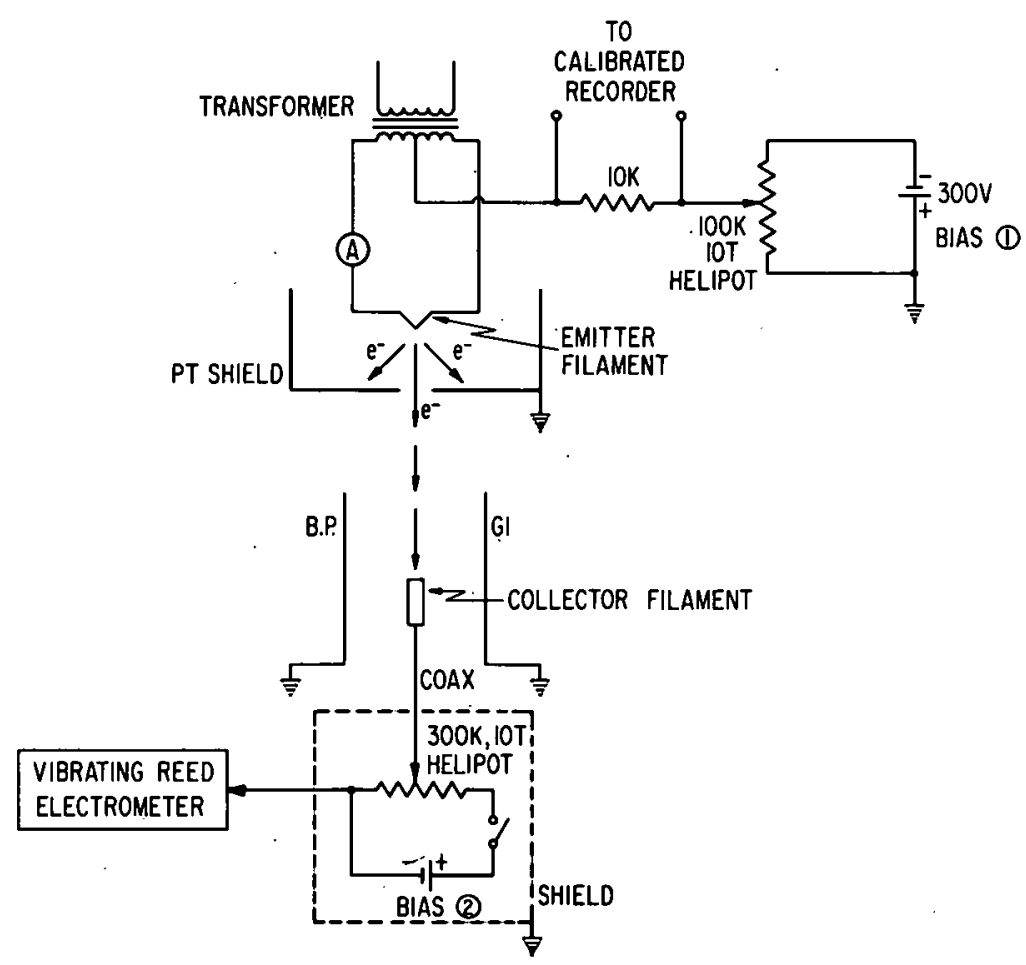

$121-5500$

Fig. 21. Schematic Diagram of Arrangement for Measurement of Thermionic WorkFunction

The emitter and collector filaments were spaced $9.05 \mathrm{~cm}$ apart each with an exposed area of $0.051 \mathrm{sq} \mathrm{cm}$. The fraction of the emitter electrons collected by the target was $1.98 \times 10^{-4}$. The slit in the platinum shield was approximately $0.5 \mathrm{~cm}$ away from the emitter filament.

The magnitude of the current density emitted depends upon the electric field at the cathode surface. The space-charge effect is due to the potential barrier at the surface of the metal. Electrons that escape have smaller kinetic energy after leaving the metal than they have inside the metal. As a result of the reduction in velocity, there is a general pileup of electrons just outside the metal surface, producing a space charge. The existence of the negative space charge causes an effective increase in the potential barrier. Sufficient cathode-anode voltage was supplied to ensure operation in the saturation emission region.

Since the applied electric field is an accelerating field, it aids the electrons in overcoming the image force at the surface of the metal and therefore lowers the work function. For the applied potentials used, the Schottky effect is negligible. For an accelerating potential of $300 \mathrm{~V}$, the work function is reduced by $0.006 \mathrm{eV}$, well within the error limit of the reported data.

The current was measured at the collector filament as a function of the emitter temperature. A least-squares fit of $\log \mathrm{I} / \mathrm{T}^{2}$ versus $5040 / \mathrm{T}$ was computed on an IBM 1620 computer over a range of $1650-2440^{\circ} \mathrm{K}$. 


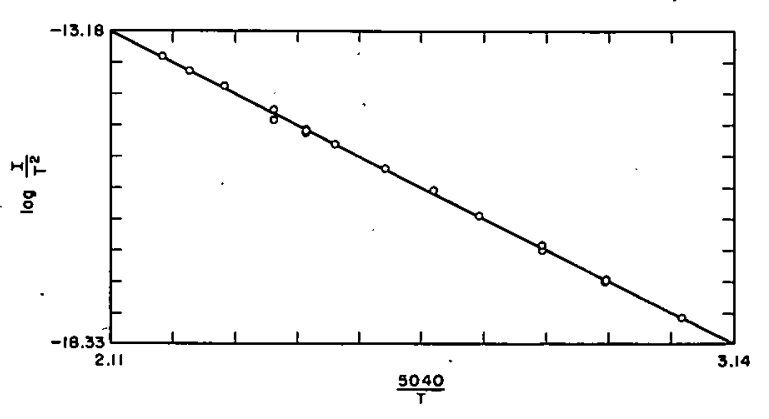

$121^{3}-5495$

Fig. 22. Thermionic Work Function of Rhenium
The result is shown in Fig. 22. The effective work function computed from the slope of the curve was $\phi_{\mathrm{e}}^{*}=$ $4.98 \pm 0.03 \mathrm{eV}$. The preexponential term, $A^{*} S G$, where $G$ is the geometry factor, was computed to be $20 \pm$ $5 \times 10^{-4}$, giving an effective thermalemission constant, $A^{*}$, of $150-$ $250 \mathrm{~A} \mathrm{~cm}^{-2}{ }^{\circ} \mathrm{K}^{-2}$. Table XI compares these results with those obtained by other workers. r

TABLE XI. Emission Constants of Rhenium

\begin{tabular}{lcc|ccc}
\hline$\phi_{\mathrm{e}}^{*}, \mathrm{eV}$ & $\mathrm{A}^{*}, \mathrm{~A} \mathrm{~cm}^{-2}{ }^{\circ} \mathrm{K}^{-2}$ & Reference & $\phi_{\mathrm{e}}^{*}, \mathrm{eV}$ & $\mathrm{A}^{*}, \mathrm{~A} \mathrm{~cm}^{-2}{ }^{\circ} \mathrm{K}^{-2}$ & Reference \\
\hline $4.98 \pm 0.03$ & $150-250$ & This work & $4.97 \pm 0.03$ & 120 & 183 \\
$4.85 \pm 0.05$ & $60 \pm 20$ & 181 & $4.96 \pm 0.05$ & $180-250$ & 184 \\
$4.96 \pm 0.05$ & - & 182 & & & \\
\hline
\end{tabular}

The first three rows refer to rhenium ribbons, and the last two rows to rhenium wires.

The quantitative variation in the thermionic work-function values is seen to be slight. This can easily be attributed to variations in the crystal structure of the rhenium ribbon or wire used by different experimenters.

The theoretical value of the thermal-emission constant,

Eq. 3.79 , is

$$
\mathrm{A}=\frac{4 \pi \mathrm{mek}^{2}}{\mathrm{~h}^{3}}=120 \mathrm{~A} \mathrm{~cm}^{-2}{ }^{\circ} \mathrm{K}^{-2}
$$

and contains only universal constants. Its value should be the same for all pure metals. This is not true experimentally.

Some reasons for the discrepancy can readily be invoked. The work function depends to a slight extent on temperature, since the ionic spacing of the metal lattice changes with temperature. A second reason has to do with the wave nature of the electron. Some of the electrons with surface-directed energies greater than the work function will not escape, but; rather, will be reflected back into the metal. This suggests that the experimentally determined emission-constant value should be less than the theoretical value. The largest effect on the value of the emission constant depends on the work function of the different crystal faces. The size and shape of the patches depend on the material, crystal size, and degree, of heat treatment. For rough surfaces, the true area can also be 
considerably larger than the geometrical area. From the above considerations, it is surprising that the experimentally determined thermal-emission constant is as uniform as it is.

\section{c. Surface-ionization Work Function}

This section describes the determination of the effective surface-ionization work function for lithium atoms on rhenium. The change in work function on adsorption of oxygen from the residual gases is also described. Chapter $\mathrm{V}$ is concerned with the surface ionization of lithium salt molecules.

Lithium metal of greater than $99 \%$ lithium isotope seven was melted directly onto a rhenium filament in an atmosphere of argon gas. The sample filament was placed in the ion source of the mass spectrometer, its position corresponding to that of the emitter filament shown in the schematic drawing of the ion source in Fig. 21. Also shown in the figure is the relative position of the surface-ionization filament. The geometry factor for the flux of particles leaving the sample filament and intercepted by the surface ionization filament was $1.98 \times 10^{-4}$. The temperature of the rhenium surface-ionization filament was measured by optical pyrometry.

From the thermodynamic properties of the sample, the vapor pressure and hence the flux of particles leaving the sample filament were determined. The thermodynamic properties were obtained from the JANAF Tables. ${ }^{100}$ Figure 23 gives the vapor pressure of lithium metal as

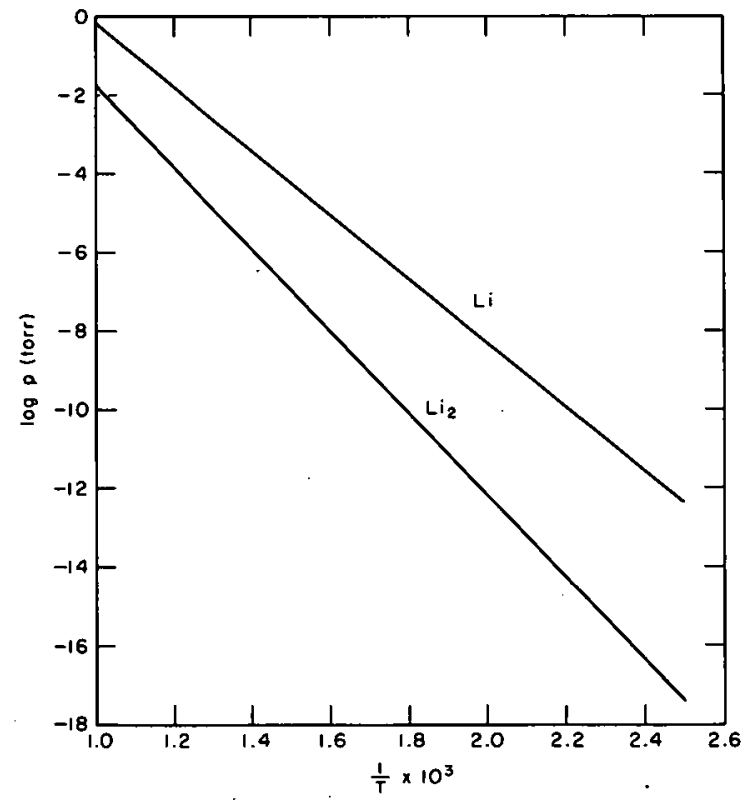

$121-5499$

Fig. 23. Vapor Pressure of Lithium Metal vs Reciprocal Temperature a function of the reciprocal temperature. The presence of dimeric lithium is negligible with respect to the monomeric species. The temperature of the sample filament was determined from Maxwell bridge resistance measurements as described in Section IV.A.3.a.

In preliminary experiments, the rhenium surface-ionization filament was heated. The background lithium present was determined by monitoring the lithium seven isotope ion current as a function of temperature. The result is presented in Fig. 24. The sharp rise in surfaceionization current was a result of boiling out lithium from the interior of the rhenium metal. Sodium, potassium, and aluminum showed 


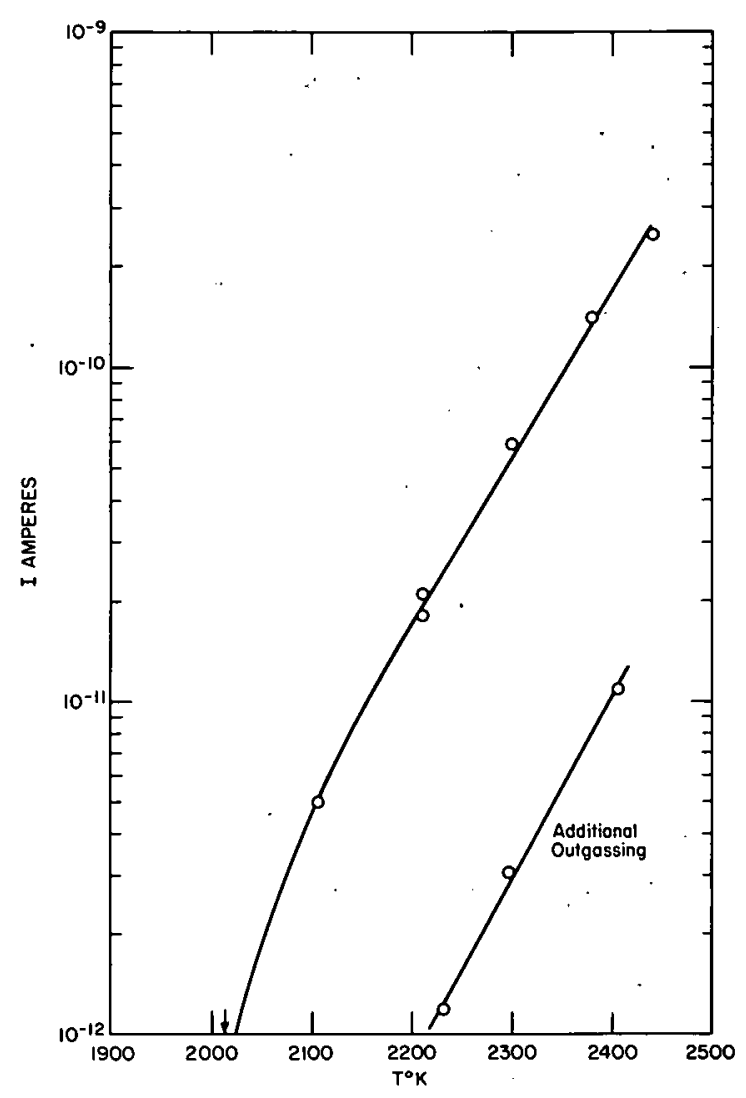

$121-5516$

Fig. 24. Background Lithium Ion Current from Rhenium Filament similar behavior. The potassium ion current was two orders of magnitude more intense than the lithium ion current.

The filament had previously been degassed and baked out to $2700^{\circ} \mathrm{K}$ in an auxiliary vacuum system. Additional heating of the filament in the ion source for several hours at $2400^{\circ} \mathrm{K}$, overnight at $2000-2100^{\circ} \mathrm{K}$, and $30 \mathrm{~min}$ at $2700^{\circ} \mathrm{K}$ reduced the background lithium ion current by more than a factor of ten (as shown in Fig. 24). This heating also markedly reduced the hydrocarbon background in the ion source.

The earlier experiments with uncollimated beams of lithium or lithium salts incident on a hot rhenium surface gave anomalous lithium ion currents that did not follow the SahaLangmuir equation. It was evident that the incident flux was changing due to thermal-radiation effects on the adjacent electrode walls. To

minimize the effect of adsorption on the electrode walls, a collimating slit was installed $1.5 \mathrm{in}$. from the sample filament. The slit size allowed the beam material to strike the surface-ionization filament, but not the adjacent electrodes.

Figure 25 shows the variation in the lithium surfaceionization current with temperature of the ionizing filament for the collimated lithium beam of constant flux $\left(5 \times 10^{11}\right.$ atoms $\left.\mathrm{cm}^{-2} \mathrm{sec}^{-1}\right)$. The plotted points represent the measured ion current within $10 \mathrm{sec}$ after flashing. The residual gas pressure was about $9 \times 10^{-9}$ Torr, as measured by the hot-cathode ionization gauge. The calculated curve corresponds to the Saha-Langmuir equation using a work function of $5.21 \mathrm{eV}$.

Below $1100^{\circ} \mathrm{K}$, the temperature was estimated from the setting of the filament Variac dial, previously calibrated against the resistance of the filament. The disparity between the calculated and observedion current with decreasing temperature is attributed to lithium adsorption on the filament, lowering the work function. No observable change in the ion current occurred after flashing. This suggests that adsorption equilibration occurred at a rate faster than the time constant of the measuring apparatus. 


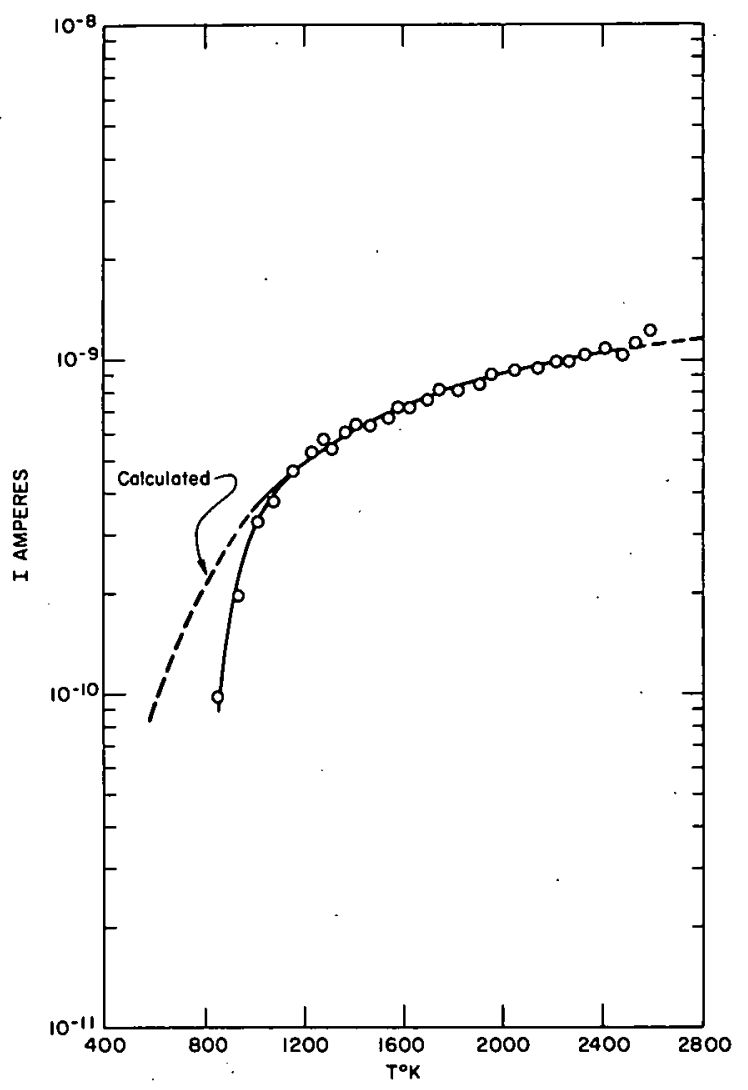

$121-5515$

Fig. 25. Temperature Dependence of ${ }^{7} \mathrm{Li}$ Ion Current
A confirmation of lithium adsorption was attempted by measuring the decrease in ion current below $1100^{\circ} \mathrm{K}$ as a function of the rate of evaporation of lithium from the sample filament. The results are inconclusive, due to the limited size of the sample. The sample was depleted before a definite trend was observed. This procedure has been used for other systems, for example, copper and silver atoms impinging on tungsten, verifying adsorption at low temperatures. ${ }^{185}$

Above $2500^{\circ} \mathrm{K}$, the ion current increased with temperature to a greater extent than predicted by the Saha-Langmuir equation. This is ascribed to an increased flux of lithium from adjacent electrodes.

The surface-ionization work function was determined in the following manner: The surfaceionization current is given by

$$
I=C A Z \beta \text {, }
$$

where $C$ is the detection efficiency of the mass spectrometer for the surface-ionization filament in ampere-seconds per atom, $Z$ is the flux impinging on the hot filament in atoms $\mathrm{cm}^{-2} \mathrm{sec}^{-1}$, $A$ is the area of the ionizing filament, and $\beta$ is the ionization coefficient. The ratio of the ion currents at two different temperatures can be used to obtain a value for the surface-ionization work function of the rhenium surface. Thus,

$$
\frac{I_{T_{1}}}{I_{T_{2}}}=\frac{\beta_{1}}{\beta_{2}}=\frac{1+2 \exp \frac{\Delta 11600}{T_{2}}}{1+2 \exp \frac{\Delta 11600}{T_{1}}}
$$

where $\Delta=I-\phi$. The value of $\Delta$ that best fits the curve drawn in the range of $1300-2400^{\circ} \mathrm{K}$ is $\Delta=0.18 \mathrm{eV}$ (dotted line in Fig. 25). This corresponds to a surface-ionization work-function value of $5.21 \mathrm{eV}$, using $5.39 \mathrm{eV}$ for the ionization potential of lithium atoms.

The effective surface-ionization work function for polycrystalline rhenium obtained by Werning ${ }^{7}$ was $5.17 \pm 0.02 \mathrm{eV}$ based on the 
surface ionization of strontium, and $5.21 \pm 0.02 \mathrm{eV}$ based on the surface ionization of barium. Zandberg and Tontegode ${ }^{8}$ obtained a value of $5.34 \cdot \mathrm{eV}$ for the effective surface-ionization work function of polycrystalline rhenium based on the surface ionization of lithium.

Direct comparison of effective surface-ionization work functions obtained by different experimentalists is difficult. The source and heat treatment of rhenium can alter the extent and type of patches obtained on a polycrystalline surface. Furthermore, the higher the ionization potential of the atom ionized the more the high-work-function patches contribute to the ionization current from polycrystalline surfaces.

The error in the work function is estimated as follows: The largest deviation in the measured current from the line drawn in Fig. 25 is $\pm 5 \%$. The deviation in the flux impinging on the hot filament was determined by measuring the surface-ionization current at the flashing temperature at intervals throughout the run. The standard deviation was $6 \%$. In addition, the sample filament temperature varied by no more than $R_{T}= \pm 0.02 \mathrm{ohm}$, corresponding to about $\pm 2^{\circ} \mathrm{K}$. The absolute value of the incident flux at $612^{\circ} \mathrm{K}$ was $4.88 \times 10^{11}$ atoms $\mathrm{cm}^{-2} \mathrm{sec}^{-1}$. The incident flux was not known to better than $\pm 25 \%$, due to the large error in the heat of formation from which the flux was determined. However, the constancy of the beam density is the critical factor.

from

The error in $\Delta$ at a given temperature may be calculated

$$
\sigma_{\Delta}=\sqrt{\left(\frac{\partial \Delta}{\partial I}\right)^{2} \cdot \sigma_{I}^{2}+\left(\frac{\partial \Delta}{\partial Z}\right)^{2} \cdot \sigma_{Z}^{2}+\left(\frac{\partial \Delta}{\partial T}\right)^{2} \sigma_{T}^{2}},
$$

where $\sigma_{I}, \sigma_{Z}$, and $\sigma_{T}$ are the standard errors in the ion current, flux, and surface-ionization filament temperature, respectively. At $1600^{\circ} \mathrm{K}$, the error in $\Delta$ is $\pm 0.01 \mathrm{eV}$. The corresponding error in the work function is likewise $\pm 0.0 \mathrm{l} \mathrm{eV}$, as suming no error in the ionization potential.

The difference between the effective surface-ionization work function ( $5.21 \mathrm{eV}$ ) and the effective thermionic work function ( $4.98 \mathrm{eV}$ ) gives a measure of the degree of contrast of the emitter surface. Patches with high work functions contribute more to the positive surface-ionization current than do low-work-function patches. For thermionic emission, the principal contribution to the electron current arises from low-workfunction patches.

d. Effect of Residual Gases on Work Function. The ion current obtained in the range of $1600-2400^{\circ} \mathrm{K}$ did not change on flashing the filament to $244 l^{\circ} \mathrm{K}$ and subsequent return to the temperature of interest. Below $1600^{\circ} \mathrm{K}$, the ion current obtained depended markedly on the residual-gas pressure. 


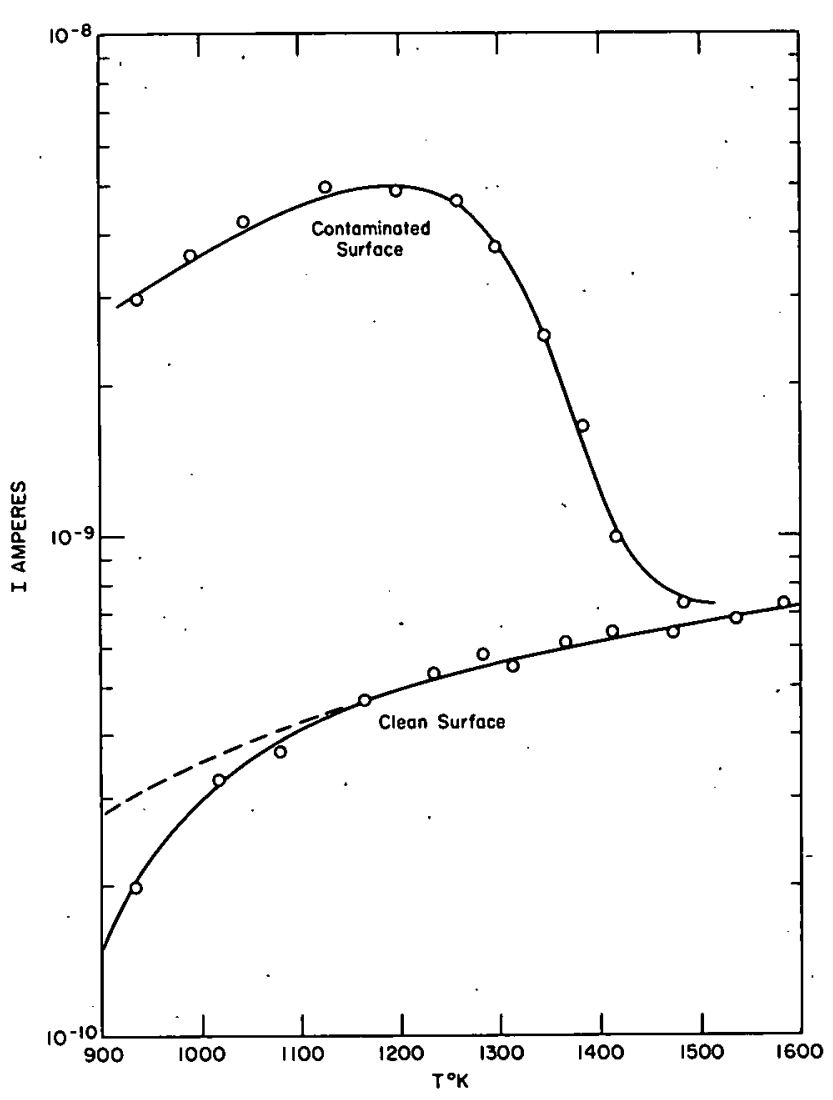

$121-5514$

Fig. 26. Temperature Dependence of Lithium Ion Current for Clean and Contaminated Surfaces
A steady-state flow of dry air was admitted to the ion source with the ion gauge reading $8.1 \mathrm{x}$ $10^{-7}$ Torr. Figure 26 shows the variation in the lithium surfaceionization current with temperature. Flashing the filament had negligible effect on the contaminatedsurface ion current obtained.

When the residual-gas pressure was lowered to $3.2 \mathrm{x}$ $10^{-8}$ Torr, the ion current immediately after flashing corresponded to the clean-surface ion current. The contaminated-surface equilibrium ion current was obtained within a few minutes after flashing. At the lowest pressure attainable, $4 \times 10^{-9}$ Torr, the time required to attain ioncurrent equilibration was of the order of hours at $1200^{\circ} \mathrm{K}$.

Electron-gun operation on the species desorbed from the contaminated surface on flashing showed high ion intensities of rhenium dioxide and rhenium trioxide. Other molecules containing lithium or rhenium were not observed. The increase in the ion intensities of residual gases such as nitrogen, carbon monoxide, and water likewise increased during flashing, due to desorption from adjacent electrodes.

The sticking coefficients of residual gases on rhenium (listed in Table X) suggest negligible adsorption of these gases at high temperatures. The principal adsorbing specie is therefore molecular oxygen, with some contribution from the oxygen present in water and carbon monoxide.

The observed lithium surface-ionization current can be rationalized as follows: At relatively high pressures of oxygen and for temperatures above $1600^{\circ} \mathrm{K}$, the equilibrium surface coverage is very low and is rapidly attained, resulting in negligible change in the work function. As the temperature decreases below $1600^{\circ} \mathrm{K}$, the equilibrium surface concentration of oxygen increases, equilibrium is attained rapidly, and the work function increases. When the oxygen pressure is reduced, the time required for the lithium surface-ionization current to attain equilibrium increases. 
Figure 27 shows the times required for the ion current to approach equilibrium after flashing for several temperatures in the range

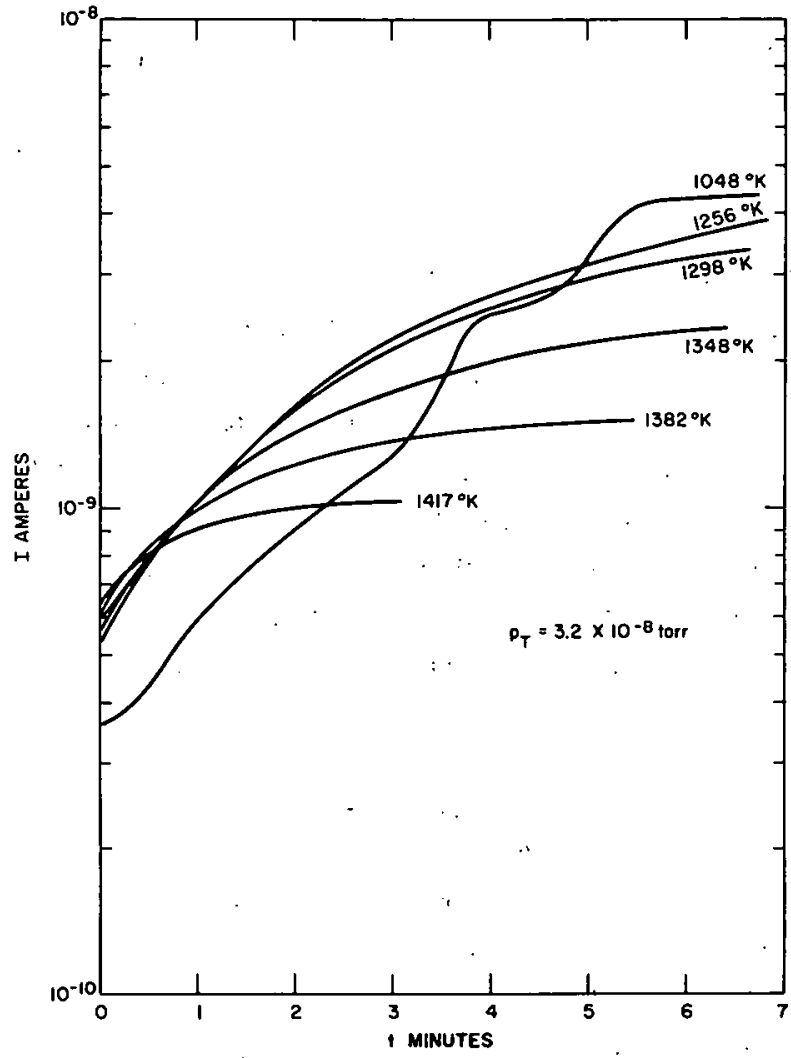

$121-5511$

Fig. 27. Approach of Lithium Ion Current to Equilibrium for Contaminated Surface of $1200-1450^{\circ} \mathrm{K}$. The total iongauge pressure was maintained at $3.2 \times 10^{-8}$ Torr. At a lower temperature $\left(1048^{\circ} \mathrm{K}\right)$, the currentversus-time curve shows considerable structure (Fig. 27). This suggests the superposition of adsorption curves corresponding to more than one type of adsorption center. It may also reflect competition for the adsorption sites between lithium and oxygen.

Above $1200^{\circ} \mathrm{K}$, the currentversus-time curves suggest Langmuir-type adsorption on a homogeneous surface. The desorption energy for oxygen on rhenium can be estimated from these curves using realistic assumptions.

The surface-ionization current obeying the Saha-Langmuir equation for a clean surface is given by

$$
I_{0}=C A Z \beta_{0}=\frac{C A Z}{1+2 \exp \frac{I-\phi}{k T}},
$$

where the symbols were previously defined. If the change in ion current for a contaminated surface is assumed to be due entirely to the change in work function, the appropriate expression is

$$
I=\operatorname{CAZ} \beta=\frac{\mathrm{CAZ}}{1+2 \exp \frac{I-(\phi+\Delta \phi)}{k T}} .
$$

From these two equations,

$$
\Delta \phi=\cdot \mathrm{kT} \cdot \ln \frac{\left(\mathrm{CAZ}-\mathrm{I}_{0}\right) \mathrm{I}}{(\mathrm{CAZ}-\mathrm{I}) \mathrm{I}_{0}}
$$


Chemisorbed atoms form dipoles on the surface of their adsorbents. The dipoles influence the work function of the metal, increasing the work function when the dipoles point with their negative poles away from the metal. The change in work function is given by

$$
\Delta \phi=\frac{\sigma \mathrm{u}}{\mathrm{E}_{0}}=\frac{\mathrm{u} \sigma_{0} \theta}{\mathrm{E}_{0}}=\Phi \theta
$$

where $\sigma$ is the surface coverage in atoms per square meter, $\sigma_{0}$ is the maximum number of atoms that can be adsorbed per square meter, $\theta$ is the degree of occupation, $u$ is the effective part of the dipole moment caused by chemisorption in coulomb-meters, and $E_{0}$ is, a constant with units of farads per meter. Since the field due to a collection of individual dipoles is negligible a few angstroms from the surface, Eq. 4.43 has some validity. It is assumed that the interaction of the adatoms can be neglected; i.e., $u$ is a constant. However, this is not quite correct, as the dipole moment is also a function of coverage, particularly at high coverage.

The rhenium surface is assumed to consist of patches of macrostructure with different emission properties. The surface can then be considered homogeneous (but not uniform), since emission from one kind of patch predominates over emission from other patches.

The increase in $\triangle \phi$ tends to a limiting value for each patch corresponding to the total coverage of the relevant type of adsorption center. Furthermore, in the chemisorption of oxygen, the metal has to provide the binding electrons, and a low value of the work function might favor this process, excluding steric factors.

For unrestricted adsorption and no dissociation, all molecules striking the surface stick, and the Langmuir adsorption isotherm may be written as

$$
\theta=\theta_{A} \frac{Z}{Z+K \exp \left(-\frac{E_{A}}{k T}\right)},
$$

where $\theta_{A}$ is the limiting value of coverage as $p \rightarrow \infty$ or $T \rightarrow 0$ for patches of type $A, Z$ is the flux of molecules of the adsorbate, $E_{A}$ is the desorption energy from patch $A$, and $K$ is a constant.

Oxygen adsorbs on metals with dissociation. ${ }^{40}$ For the adsorption process, the flux of molecules in the adsorption isotherm is replaced by $Z^{1 / 2}$. Furthermore, the Langmuir is otherm is valid only for a constant adsorption energy. Adsorption energies decrease with coverage. This can be rationalized by subdividing the surface into adsorbing centers 
of differing strengths and no mutual effects. Since the adsorption centers with high energies are occupied first, the adsorption energy drops as coverage increases.

tion is given by

For each type of adsorption center, the net rate of adsorp-

$$
\frac{\mathrm{d} \theta}{\mathrm{dt}}=\mathrm{k}_{1}(1-\theta)-\mathrm{k}_{2} \theta
$$

where $k_{1}=f(Z, T) / \sigma_{0}$ and is only slightly dependent on temperature. $r$ The rate constant,

$$
k_{2}=\frac{K}{\sigma_{0}} \exp \left(\frac{-E_{A}}{k T}\right)
$$

is markedly dependent on temperature, with $\mathrm{K}$ constant. The integrated form of the rate equation then gives the approach to equilibrium as

$$
\theta=\theta(Z, T)\left\{1-\exp \left[-\left(k_{1}+k_{2}\right) t\right]\right\}
$$

Combining this equation with Eqs. 4.42 and 4.43 gives

$$
\begin{aligned}
\Delta \phi= & k T \ln \frac{\left(\mathrm{CAZ}-\mathrm{I}_{0}\right) I}{(\mathrm{CAZ}-\mathrm{I}) \mathrm{I}_{0}}=\Phi \theta \\
= & \Phi \theta(\mathrm{Z}, \mathrm{T})\left\{1-\exp \left[-\left(\mathrm{k}_{1}+\mathrm{k}_{2}\right) \mathrm{t}\right]\right\} . \\
& \text { For any given temperature, } \\
\Delta \phi= & \Delta \phi_{\mathrm{M}}\left\{1-\exp \left[-\left(\mathrm{k}_{1}+\mathrm{k}_{2}\right) \mathrm{t}\right]\right\} .
\end{aligned}
$$

Since $\Delta \phi_{\mathrm{M}}$ and the variation of $\Delta \phi$ with time are known, $\mathrm{k}_{1}+\mathrm{k}_{2}$ can be obtained as a function of the temperature. It is apparent from Eq. 4.46 that at higher temperatures, $k_{2}>k_{1}$, while at lower temperatures, $k_{1}$ predominates. Figure 28 is a plot of $k_{1}+k_{2}$ versus temperature.

The slope at higher temperatures corresponds to a desorption energy for oxygen atoms of $E_{A}=4.12 \mathrm{eV}$. A second measure of $E_{A}$ can be obtained from the point where $k_{2}=k_{1}$. From Fig. $28, k_{2}=k_{1}$ at $1354^{\circ} \mathrm{K}$. The Polanyi-Wigner theory ${ }^{40}$ of desorption assumes that any particle possessing the requisite activation energy desorbs within the period of one vibration perpendicular to the surface. For a chemical bond, 


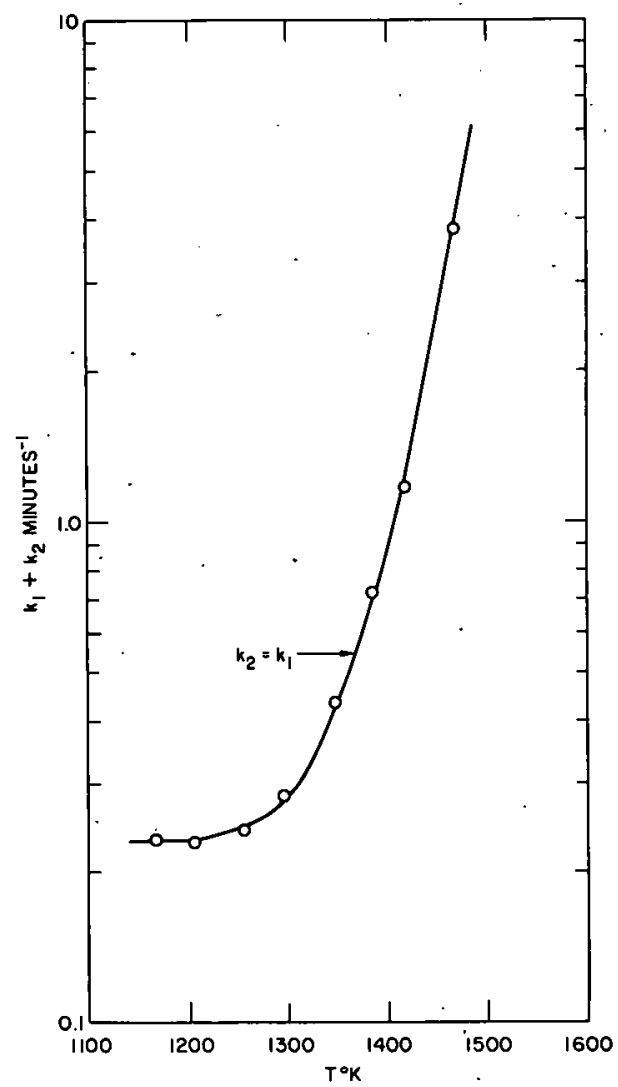

$121-5512$

Fig. 28. Temperature Dependence of Composité Rate Constants.

$$
\frac{K}{\sigma_{0}}=\nu \cong 10^{13} \mathrm{sec}^{-1}
$$

Using this result in Eq. 4.46 yields $E_{\dot{A}}=$ $4.13 \mathrm{eV}$.

An error analysis on the value obtained for $E_{A}$ is not warranted. Many assumptions of some validity were used to arrive at a desired result. A more involved analysis, taking into account the variation of $\mu, E_{A}$, and $K$ with coverage, could perhaps lead to a more exact description of the effect of oxygen adsorption on the work function of rhenium. This is beyond the scope of this report. It is also pertinent to question whether the term chemisorption is a fitting designation to apply to the adsorption of oxygen on metals. In the desorption process, oxygen is removed in large measure as the metal oxide.

The results presented illustrate a method of approach to obtain the desorption energy of gases on metals by surfaceionization techniques. Of more importance in surface-ionization work is the necessity for a clean system under highvacuum conditions. The validity of the Saha-Langmuir equation over a. wide range of temperature has been demonstrated for the lithium-rhenium system. This has not been obtained for other systems or by other investigators. 


\section{CHAPTER V}

\section{RESULTS AND DISCUSSION}

The surface ionization of molecular beams of lithium chloride, lithium bromide, lithium iodide, lithium sulfate, and lithium nitrate are discussed in this chapter. Beams of lithium salt impinge on a hot rhenium surface, and the temperature dependence of the lithium surface-ionization current is measured. The results are compared with those obtained for pure atomic beams of lithium atoms. Deviations are analyzed with respect to the effect of the anionic constituent in the molecular beam. These experiments provide a method for determining the dissociation energies of the gaseous molecules where conditions are favorable.

\section{A. The System Lithium Chloride on Rhenium}

Up to $1 \mathrm{mg}$ of lithium chloride (m.p. $883^{\circ} \mathrm{K}$ ) from an aqueous solution was evaporated onto a sample filament. The vapor pressure of lithium

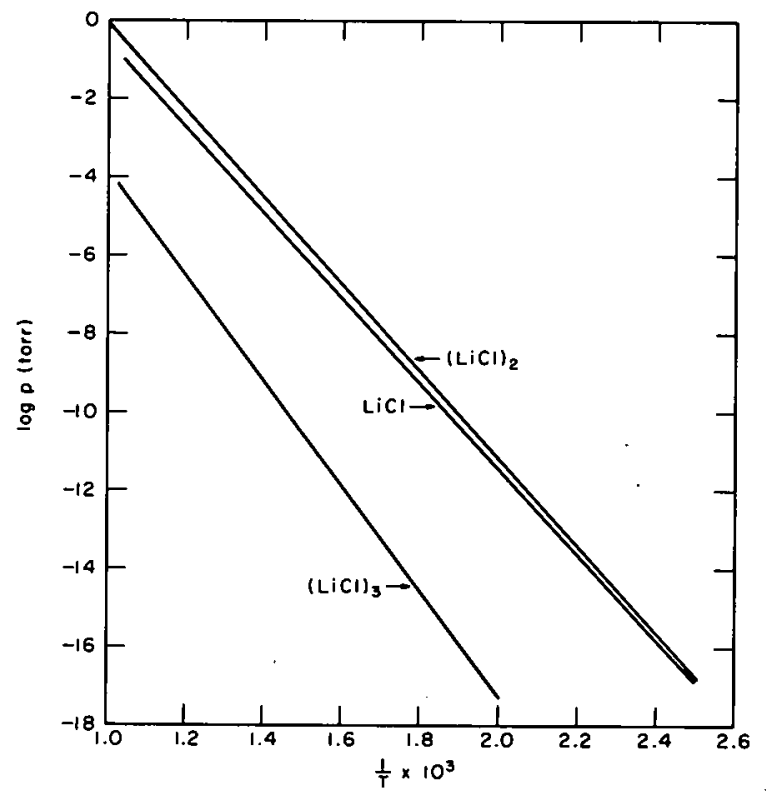

$121-5505$

Fig. 29. Vapor Pressure of Lithium Chloride vs Reciprocal Temperature chloride, and hence the flux of particles leaving the sample filament, was. obtained from the thermodynamic properties listed in the JANAF Tables. ${ }^{100}$ Figure 29 shows the vapor pressure as a function of the reciprocal temperature. For lithium halides, the dimeric species is present in greate $r$ abundance than the monomer species in the vapor phase. The abundances of the trimeric species was negligible in these experiments.

Total incident fluxes of $1.97 \times 10^{11}$ and $3.29 \times 10^{12}$ molecules $\mathrm{cm}^{-2} \mathrm{sec}^{-1}$ were calculated from the temperature measurements of the sample filament. Twice the lithium chloride dimer flux is included in the total flux. From the rmodynamic arguments, the dimer dissociates to monomer at all ionizing filament

temperatures. The total flux impinging may therefore be considered to consist of the monomeric species in these experiments.

Figure 30 shows the temperature dependence of the lithium ion current due to surface ionization of lithium chloride molecules for the two different fluxes. Each dashed line corresponds to the current expected for complete dissociation and subsequent surface ionization of lithium atoms 


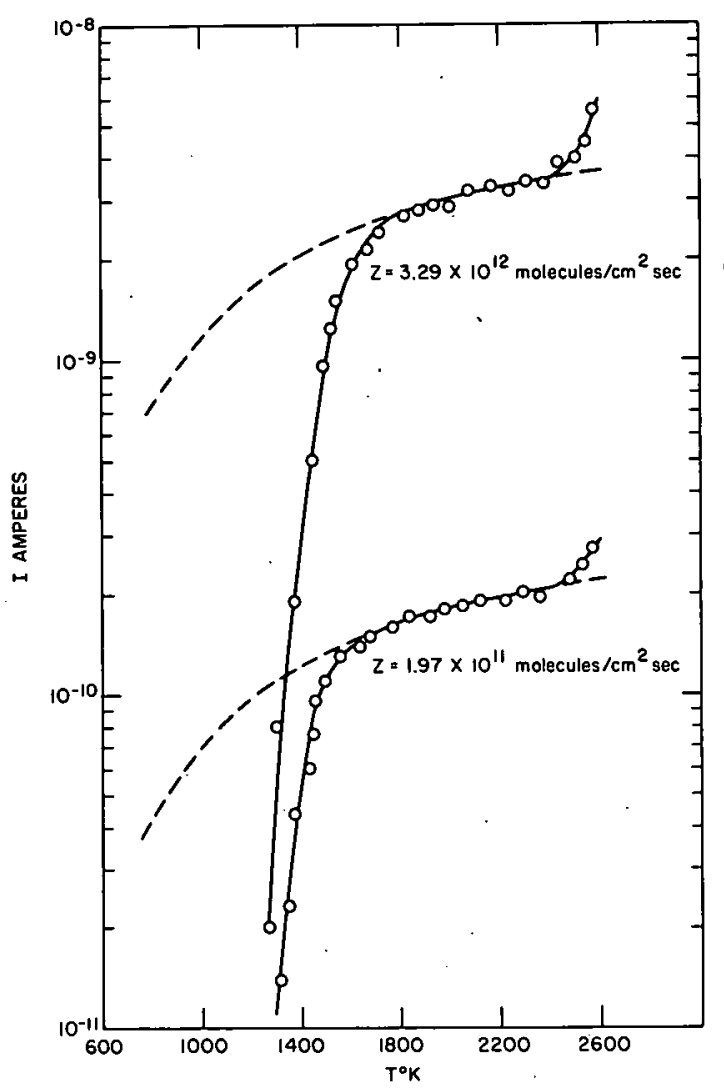

$121-5513$

Fig. 30. Temperature Dependence of the Surface Ionization of Lithium Chloride using the experimental ionization coefficient previously determined for an incident flux of lithium atoms.

Above $2400^{\circ} \mathrm{K}$, the increase in ion current with temperature is again attributed to desorption from the adjacent electrodes. This is more pronounced with the greater incident flux. The threshold for surface ionization of lithium chloride occurred at about $1200^{\circ} \mathrm{K}$, some $400^{\circ} \mathrm{K}$ above that observed for lithium atoms.

The fluxes were constant to within $6 \%$, both by observation of the variation in sample filament resistance and from the measured surface-ionization current at $2300^{\circ} \mathrm{K}$ before and after the run. The ratio of the incident fluxes agrees with the ratio of the measured ion currents from the surface-ionization filament in the range of $1700-2300^{\circ} \mathrm{K}$. The principal ionic masses observed by electron-impact ionization correspond to $\mathrm{Li}^{+}(6,7), \mathrm{LiCl}^{+}(42,44)$, and $\mathrm{Li}_{2} \mathrm{Cl}^{+}$ $(48,49,51)$. The residual-gas pressure was $8.2 \times 10^{-9}$ Torr.

Above $1500^{\circ} \mathrm{K}$, flashing the filament before each current measurement had no detectable effect on the surface ionization current. The surface was therefore clean. Below $1500^{\circ} \mathrm{K}$, the ion current was obtained immediately after flashing. The equilibrium ionization current was approached slowly, of the order of hours. This is in agreement with the discussion in Section IV.B.2.d on the effect of low residual-gas pressures on the work function.

The sharp dropoff in the ion current in the region near threshold is attributed to the incomplete dissociation of the lithium chloride, the molecules reevaporating. For greater incident fluxes, the decrease in ion current near the threshold region occurs at higher temperatures, as shown in Fig. 31.

At high incident beam intensities or lower temperatures, a significant fraction of the molecules may be desorbed from the surface. As shown in Section III.D, it should be possible to obtain the dissociation energy of lithium chloride if certain conditions are met. 


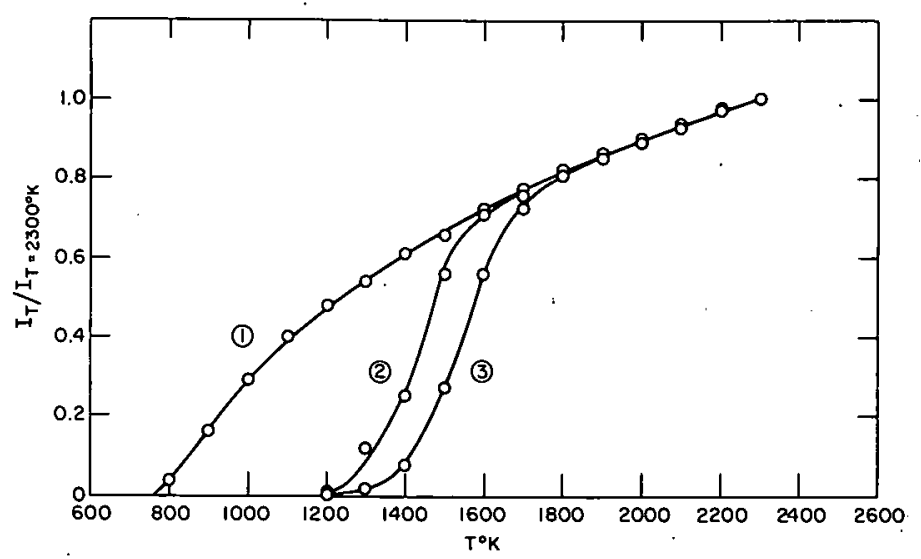

Fig. 31

Temperature Dependence of Surface Ionization on Rhenium. Curve 1: Lithium atoms; Curve 2: Low-incident LiCl flux, and Curve 3: High-incident LiCl flux.

The relationship between the dissociation energy and the ionization coefficients is given by Eq. 3.52,

$$
x_{1} z_{1}=\frac{\beta^{2}}{1-\beta} \cdot \frac{\beta_{1}-\beta_{2}}{\beta_{1} \beta_{2}} \cdot \frac{1}{c \beta_{2}-\beta_{1}}
$$

with

$$
\mathrm{x}_{1}=\mathrm{C}_{1} \exp \frac{\mathrm{D}_{\mathrm{MX}}(\mathrm{g})}{\mathrm{kT}}
$$

where $Z_{1}$ is the smaller incident flux, $\beta$ is the ionization coefficient for atoms, $\beta_{1}$ and $\beta_{2}$ are the ionization coefficients for fluxes $Z_{1}$ and $Z_{2}$, respectively, and $K$ is defined by $Z_{2}=K Z_{1}$. The symbol $C_{1}$ is a constant, and $D_{M X(g)}$ is the dissociation energy of the molecule MX. The assumptions are that an insignificant number of chlorine molecules are produced and that the atomic ionization coefficient $\beta$ does not depend on the incident beam intensity in the threshold region for surface ionization of molecules.

In terms of ion currents, given by Eq. 4.38 , Eq. 3.52 may be rewritten as

$$
x_{1}=\frac{\beta^{2}}{1-\beta} \cdot \frac{K I_{1}-I_{2}}{I_{1} I_{2}} \cdot \frac{(C A)^{2} Z_{1}}{I_{2}-I_{1}}
$$

Figure 32 is a plot of $\log x_{1}$ versus $5040 / T$, the slope of the line giving directly the dissociation energy of lithium chloride. The value obtained for the dissociation energy in the range of $1300-1700^{\circ} \mathrm{K}$ is $\mathrm{D}_{\mathrm{MX}}(\mathrm{g})=$ $4.9 \pm 0.1 \mathrm{eV}$, compared to a literature value of $5.02 \pm 0.13 \mathrm{eV} .{ }^{100}$

Zandberg and Tontegode ${ }^{8}$ performed additional experiments on this system. They compared the surface-ionization current of indium $(I=5.785 \mathrm{eV})$ a's a function of temperature in the presence and absence of 


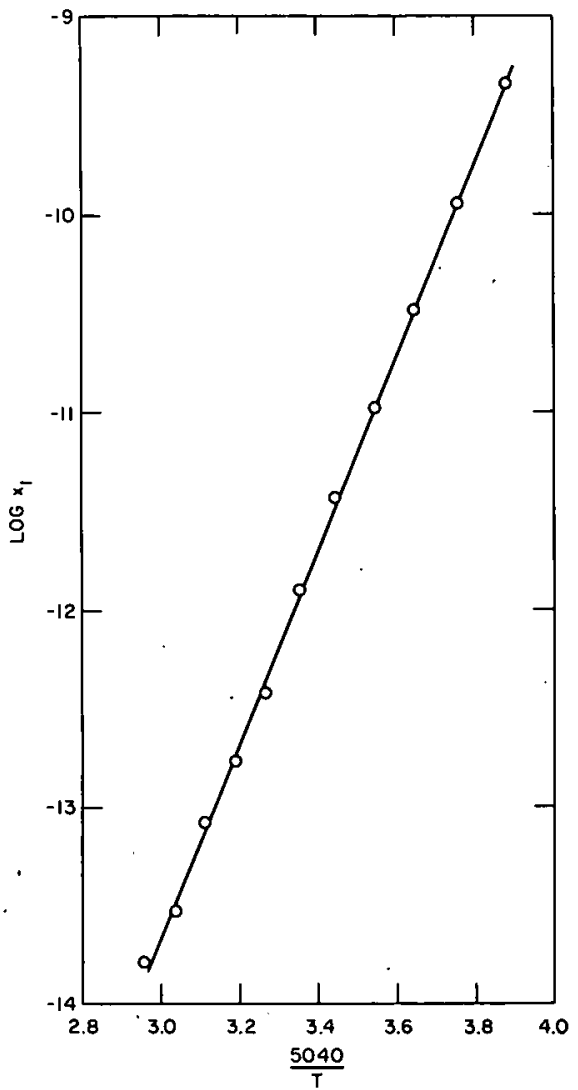

$121-5510$

Fig. 32. Dissociation-energy Data for Lithium Chloride a molecular beam of lithium chloride. In the presence of lithium chloride, the indium ion current decreased as the temperature decreased below $1650^{\circ} \mathrm{K}$. The reduction in indium ion current was ascribed to a change in the work function only. The calculated change in work function cannot account for the large reduction in the lithium ion current observed in the threshold-temperature region for surface ionization of lithium chloride molecules.

Zandberg and Tontegode ${ }^{8}$ also showed that in the combined surface ionization of lithium atoms with lithium chloride molecules, the total lithium ion current is the sum of the currents corresponding to the atomic and molecular-beam densities. If lithium chloride molecules were completely dissociated on rhenium, one would expect a significant difference in the total ion current in the threshold-temperature region for atomic lithium in comparison with the ion current obtained from an atomic beam only.

The effect of a beam of cesium chloride molecules on surface ionization of lithium atoms was also investigated. ${ }^{8}$ In the threshold-temperature region for lithium-atom surface ionization, cesium chloride is completely dissociated. When the cesium chloride beam density was 20 times as great as the lithium-atom beam density, the lithium ion current was approximately halved, indicative of association of lithium atoms for chlorine adatoms on the surface.

The difference in the ionization threshold temperatures for atomic lithium and lithium chloride molecules must be attributed to the rate of emission of molecules from the surface, being greater than the rate of dissociation of molecules on the surface. The reduction in current at the threshold region for lithium chloride beams is associated with a nearly constant value of the work function and depends on the incident flux density. From these results, the dissociation energy of gaseous lithium chloride was determined. The similar temperature dependence of the surfaceionization currents for atomic lithium and molecular beams of lithium chloride in the high-temperature region $\left(>1700^{\circ} \mathrm{K}\right)$ indicates complete dissociation of the molecules on rhenium. 
B. The System Lithium Bromide on Rhenium

Figure 33 gives the lithium ion current due to surface ionization of lithium bromide molecules (m.p. $823^{\circ} \mathrm{K}$ ). The molecular-beam intensities

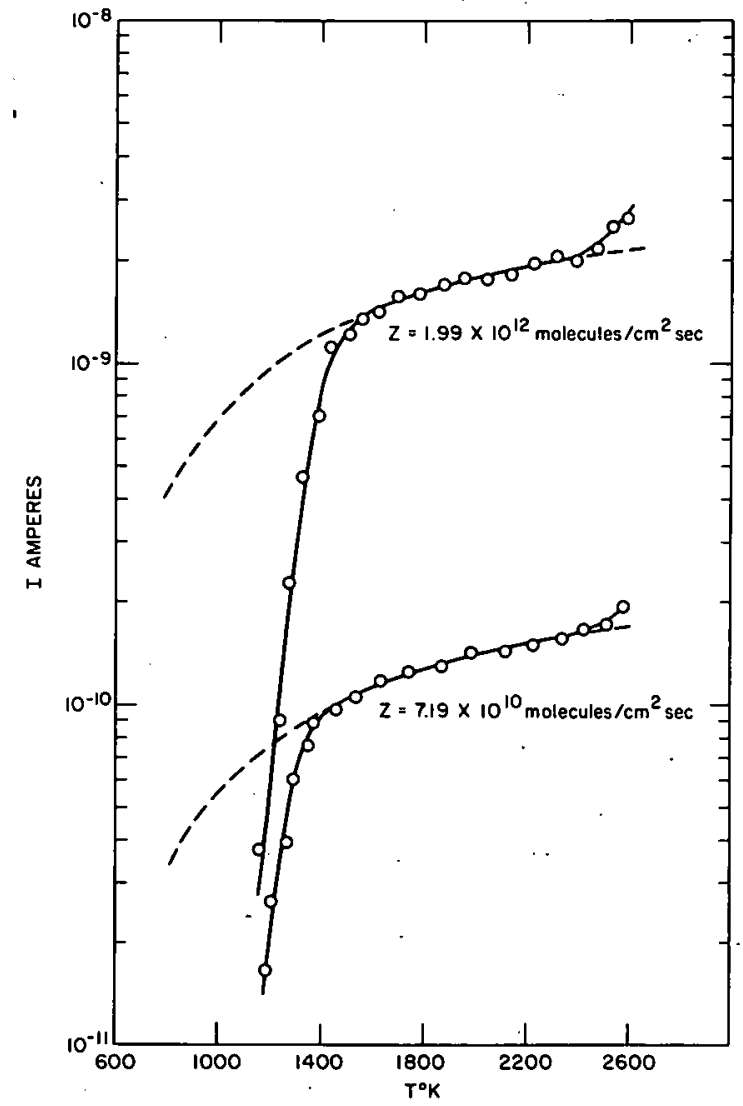

$121-5509$

Fig. 33. Temperature Dependence of the Surface Ionization of Lithium. Bromide at the surface-ionization filament were $7.19 \times 10^{10}$ and $1.99 \times 10^{12}$ molecules/ $\mathrm{cm}^{2}=\mathrm{sec}$. The reduction in lithium ion current for surface ionization of lithium bromide molecules begins at $T \cong 1400$ $1500^{\circ} \mathrm{K}$, depending on the molecularbeam intensity. This is about $200^{\circ} \mathrm{K}$ below that observed for beams of lithium chloride. The principal ionic masses observed by electron-impact ionization of the molecular beam correspond to $\mathrm{Li}^{+}(6,7), \mathrm{LiBr}^{+}(86,88)$, and $\mathrm{Li}_{2} \mathrm{Br}^{+}(93,95)$. The residual-gas pressure during the larger flux run was $8.3 \times 10^{-9}$ Tor $\mathrm{r}$ and consisted mainly of $\mathrm{H}_{2} \mathrm{O}, \mathrm{N}_{2}, \mathrm{CO}, \mathrm{O}_{2}$, and $\mathrm{CO}_{2}$.

The mass-spectrometer detection efficiency was not the same for the different incident fluxes used. It was necessary to break vacuum and replenish the lithium bromide on the sample filament. After pumping down the spectrometer and retuning the electronics, we found the detection efficiency to be different by a factor of two. The residual-gas pressure during the lower molecular-beam density run was about $8 \times 10^{-9}$ Torr.

The relationship between the dissociation energy and ion currents for two different detection efficiencies, $C_{1}$ and $C_{2}$, can be written as

$$
x_{1}=\frac{\beta^{2}}{1-\beta} \cdot \frac{K I_{1}-\frac{C_{1}}{C_{2}} I_{2}}{I_{1} I_{2}} \cdot \frac{C_{1} C_{2} A^{2} Z_{1}}{\frac{C_{1}}{C_{2}} I_{2}-I_{1}},
$$

using Eqs. 3.52 and 4.38. The plot of $\log \mathrm{x}_{1}$ versus $5040 / \mathrm{T}$ is shown in Fig. 34. The dissociation energy obtained from the slope of the line in the range of $1150-1400^{\circ} \mathrm{K}$ is $\left.\mathrm{D}_{\mathrm{LiBr}} \mathrm{g}\right)=4.4 \pm 0.1 \mathrm{eV}$, compared to the literature value of $4.47 \pm 0.13 \mathrm{eV}^{100}$ 


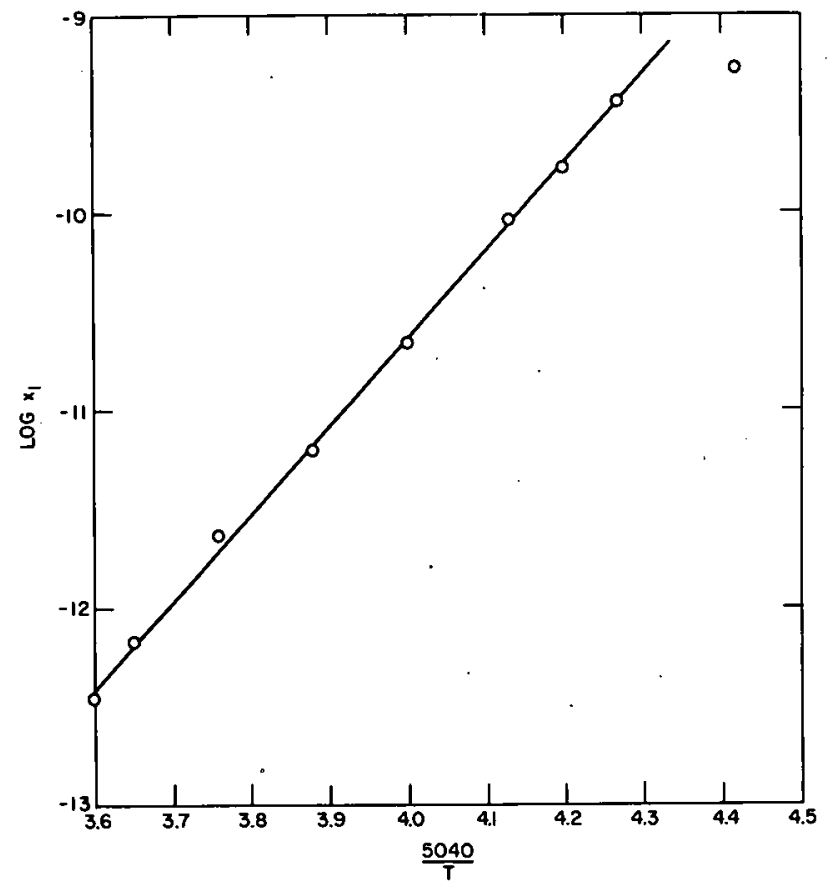

$121-5493$

Fig. 34. Dissociation-energy Data for Lithium Bromide
Below $1150^{\circ} \mathrm{K}$, the slope of $\log x_{1}$ versus the reciprocal temperature appears to approach zero. Such a result is not unexpected. At these temperatures, the ion current for lithium atomic beams does not follow the SahaLangmuir equation. The ion current is suppressed because lithium adsorption lowers the work function.

The above results were obtained from the measured current within a few seconds after the surface-ionization filament had been flashed to $\sim 2400^{\circ} \mathrm{K}$. Above $1400^{\circ} \mathrm{K}$, flashing had an insignificant affect on the lithium ion current. Below $1400^{\circ} \mathrm{K}$, the lithium ion current increased slowly with time after flashing, approaching the steady-state value. For nearly identical temperatures, the rate of increase in the lithium ion current with time is similar for both incident fluxes. The residual-gas pressure was approximately the same in each case, whereas the incident fluxes of lithium bromide differed by a factor of 28. Therefore, the increase in current is due to an increase in the work function as a result of the adsorption of residual gases, mainly oxygen. If the work function was markedly influenced by bromine adsorption, the rate of increase in ion current with time should be more pronounced for the higher incident flux. Observation of the electron-impact spectra on flashing showed no detectable rhenium bromide molecules.

\section{The System Lithium Iodide on Rhenium}

Molecular-beam intensities of lithium iodide (m.p. $742^{\circ} \mathrm{K}$ ) impinging on the surface-ionization filament were calculated from the temperature measurements of the sample filament together with the thermodynamic properties for lithium iodide listed in the JANAF Tables. ${ }^{100}$ Total incident fluxes of $5.62 \times 10^{10}$ and $1.55 \times 10^{12}$ molecules $\mathrm{cm}^{-2} \mathrm{sec}^{-1}$ were obtained for sample filament temperatures of 553 and $609^{\circ} \mathrm{K}$, respectively. Twice the lithium iodide dimer flux is included in the total flux.

The principal ionic masses observed by electron-impact ionization of the molecular beams were $\operatorname{Li}^{+}(6,7), \mathrm{LiI}^{+}(133,134)$, and $\mathrm{Li}_{2} \mathrm{I}^{+}(140,141)$. The ion $\mathrm{Li}_{3} \mathrm{I}_{2}{ }^{+}$was also detected in small abundance. In addition, ion masses corresponding to $\mathrm{I}^{+}(127), \mathrm{HI}^{+}(128)$, and $\mathrm{I}_{2}^{+}(254)$, were present in the 


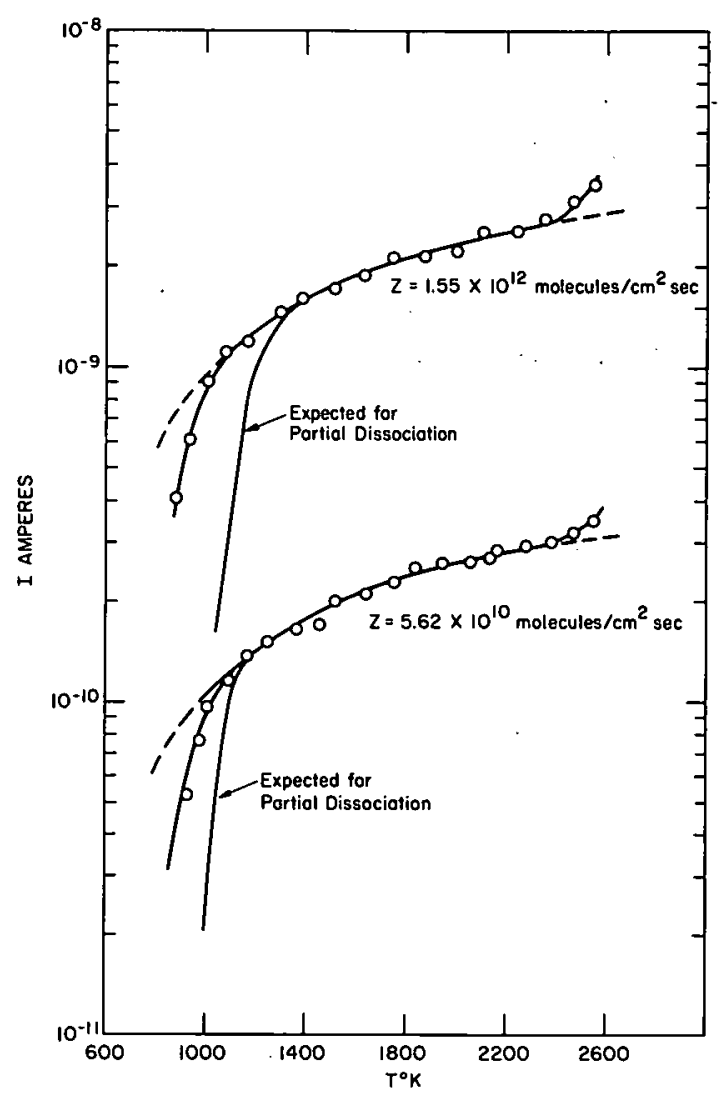

$121-5508$

Fig. 35. Temperature Dependence of the Surface Ionization of Lithium Iodide molecular beams to a small extent. The residual-gas pressure was $9.2 \mathrm{x}$ $10^{-9}$ Torr.

Figure 35 shows the temperature dependence of the lithium ion current from surface ionization of lithium iodide molecules. In addition to the SahaLangmuir curves (dashed lines), curves for the expected reduction in lithium ion current due to incomplete dissociation of lithium iodide are shown. These latter curves are based on the thermodynamic arguments presented in Chapter III. The fraction of lithium iodide dissociated at any given temperature was obtained from Eq. 3.81. The undissociated fraction was assumed to desorb as molecules.

Comparison of the temperature dependence of the surface-ionization current for lithium atoms (Fig. 25) and lithium iodide molecules (Fig. 35) indicate that lithium iodide is dissociated over the entire range of measured temperature. The decrease in the measured

lithium ion current from the Saha-Langmuir curve with decreasing temperature below $1100^{\circ} \mathrm{K}$ is similar for both lithium iodide molecules and lithium atoms incident on the surface-ionization filament. This effect was ascribed to adsorption of lithium atoms on the rhenium surface causing a decrease in the work function.

The enhanced lithium ion emission at temperatures at which incomplete dissociation is expected may be rationalized as follows: By inference from the values presented in Table $V$, a large increase in work function is required to increase the fraction of lithium iodide dissociated at a given temperature for a given flux. Such a large increase in workfunction $(\sim 1.0 \mathrm{eV})$ is unrealistic and is not found. The ion currents plotted in Fig. 35 were obtained immediately after flashing the surface-ionization filament to high temperatures and subsequent return to the temperature of interest. The fraction of the surface covered by lithium iodide molecules is a maximum of 0.01 , assuming that all molecules striking stick.

Furthermore, at $1181 \pm 5^{\circ} \mathrm{K}$, the rate of increase in ion current with time after flashing was similar for both fluxes of lithium iodide incident 
on the surface-ionization filament. Thesefluxes differed by a factor of 30 . Since the residual-gas pressure was practically the same in each instance, the increase in ion. current is a result of oxygen adsorption, increasing the work function.

A more likely explanation for the enhanced lithium ion emission is the displacement of the dissociation equilibrium towards lower temperatures by a competing reaction. The formation of $I_{2}(g)$ by coalescence of iodine atoms on the surface with subsequent evaporation. can be rejected by the thermodynamic arguments given in Chapter III. In addition, electron impact ionization of the species desorbed on flashing showed no ${ }_{2}^{+}(\mathrm{g})$ above that present in the incident beams.

Scheer and Fine ${ }^{48}$ suggested an iodide-rhenium interaction to explain their results for the mean residence times of cesium ions on rhenium for an incident beam of cesium iodide. An attempt to verify the formation of a rhenium iodide for incident beams of lithium iodide was not successful. The lithium ion current was allowed to attain equilibrium with the surfaceionization filament at $1167^{\circ} \mathrm{K}$. The filament was then flashed to $2400^{\circ} \mathrm{K}$, and the desorbed species analyzed by electron-impact ionization. Oxides of rhenium were observed, but no rhenium iodides or oxyiodides could be detected. This does not, however, rule out the possibility of a rhenium iodide unstable intermediate, which decomposes on heating the filament to high temperatures. The resulting shift of the lithium iodide dissociation equilibrium to lower temperatures can then give rise to enhanced lithium ion emission.

D. The System Lithium Sulfate on Rhenium

Knudsen effusion studies of lithium sulfate have been performed. Buchler and Berkowitz-Mattuch ${ }^{186}$ found the following:ion intensities corresponding to $\mathrm{Li}_{2} \mathrm{SO}_{4}(149), \mathrm{Li}_{2} \mathrm{SO}_{3}(1), \mathrm{Li}_{2} \mathrm{SO}_{2}(0.6), \mathrm{Li}_{2} \mathrm{O}(80), \mathrm{LiO}(1.2)$, $\mathrm{SO}_{3}(1.3), \mathrm{SO}_{2}(860)$, and $\mathrm{SO}(80)$ by mass-spectrometric techniques. The Knudsen effusion cell was at $877^{\circ} \mathrm{C}$.

The high-temperature equilibrium vapor species over solid lithium sulfate heated. in an alumina- or gold-lined Knudsen cell were investigated mass-spectrometrically by Margrave et al. ${ }^{187}$ The relative ion-intensity data at $1090^{\circ} \mathrm{K}$ for $50-\mathrm{eV}$ electrons are as follows: $\mathrm{Li}_{2} \mathrm{SO}_{4}(<0.01)$, $\mathrm{Li}_{2} \mathrm{O}(<0.01), \mathrm{Li}(3.1), \mathrm{SO}_{2}(37), \mathrm{SO}_{3}(0.5)$, $\mathrm{SO}(30)$, and $\mathrm{O}_{2}(5.5)$. Appearance potential studies. indicated that $\mathrm{Li}, \mathrm{O}_{2}, \mathrm{SO}_{2}$, and $\mathrm{SO}_{3}$ are parent species. In contrast to the work of Buchler and Berkowitz-Mattuch, ${ }^{186}$ no lithium sulfate or lithium oxide gaseous species were observed. On the basis of the evidence presented, they concluded that the vaporization of lithium sulfate is properly described by the decomposition reaction

$$
\mathrm{Li}_{2} \mathrm{SO}_{4}(\mathrm{~s})=2 \mathrm{Li}(\mathrm{g})+\mathrm{SO}_{2}(\mathrm{~g})+\mathrm{O}_{2}(\mathrm{~g}),
$$


and by the gaseous equilibrium

$$
\mathrm{SO}_{2}(\mathrm{~g})+\frac{1}{2} \mathrm{O}_{2}(\mathrm{~g})=\mathrm{SO}_{3}(\mathrm{~g})
$$

The absence of $\mathrm{Li}_{2} \mathrm{O}(\mathrm{g})$ supports the decomposition reaction written.

A second-law treatment of reaction 5.3 yielded a heat of decomposition of $324 \pm 12 \mathrm{kcal}$ mole $\mathrm{e}^{-1}$ at $1087^{\circ} \mathrm{K}$. Heat-capacity data for lithium sulphate at high temperatures are not available, so that the heat of decomposition cannot be calculated for comparison.

Results of a vaporization weight-loss study on lithium sulfate by Spitsyn and Shostak ${ }^{188}$ indicated that vaporization was due largely to decomposition into $\mathrm{SO}_{2}(\mathrm{~g})$ and $\mathrm{O}_{2}(\mathrm{~g})$. Substantial quantities of $\mathrm{Li}_{2} \mathrm{O}(\mathrm{g})$ were vaporized as well. Other mass-spectrometric studies ${ }^{189}$ have established the existence of gaseous $\mathrm{Li}_{2} \mathrm{O}(\mathrm{g})$ and $\mathrm{LiO}(\mathrm{g})$ in a ratio of $12: 1$, respectively. Knudsen-cell temperatures greater than $1200^{\circ} \mathrm{K}$ were required for their observation.

In the following experiment, approximately $1 \mathrm{mg}$ of lithium sulfate from an aqueous solution was evaporated onto a sample filament. A new rhenium surface-ionization filament was installed and baked out for several days by the procedure given in Section. IV.A.2. The sample filament was heated electrically to about $500^{\circ} \mathrm{K}$, and the beam particles emitted were analyzed by electron-impact ionization. Large intensities of ion masses corresponding to $\mathrm{SO}_{2}^{+}, \mathrm{SO}^{+}$, and $\mathrm{O}_{2}^{+}+\mathrm{S}^{+}$were observed. The relative ion abundances were ${ }^{32} \mathrm{SO}_{2}(100),{ }^{34} \mathrm{SO}_{2}(5),{ }^{32} \mathrm{SO}(49),{ }^{34} \mathrm{SO}(2)$, and $\mathrm{O}_{2}(12)$. The ${ }^{32} \mathrm{~S}$ ion intensity was less than $10 \%$ of the $\mathrm{O}_{2}$ ion. intensity based on the ${ }^{34} \mathrm{~S}$ ion intensity.

When the sample-filament temperature was raised to $900^{\circ} \mathrm{K}$, lithium ions were detected. The sulfur dioxide was in such abundance as to scram the apparatus $\left(10^{-5}\right.$ Torr). Ion masses corresponding to $\mathrm{SO}_{3}$ or $\mathrm{Li}_{2} \mathrm{SO}_{4}$ were not observed. When appreciable $\mathrm{SO}_{2}$ had evaporated, the sample-filament temperature was raised to about $1250^{\circ} \mathrm{K}$. Ion masses corresponding to $\mathrm{Li}(6,7)$ and $\mathrm{Li}_{2} \mathrm{O}(29,30)$ were present. The ion-mass ratio of $30: 29$ was 4.7 , compared to a theoretical expectation of 6.6. A residual-gas ion peak was present at mass 29 , contributing to the mass 29 ion. intensity. Ions corresponding to $\mathrm{LiO}$ were not detected.

The ion masses observed during the gradual raising of the temperature support the decomposition reaction

$$
\mathrm{Li}_{2} \mathrm{SO}_{4}(\mathrm{~s})=2 \mathrm{Li}(\mathrm{s})+\mathrm{SO}_{2}(\mathrm{~g})+\mathrm{O}_{2}(\mathrm{~g})
$$


at the lowest temperatures. At intermediate temperatures, the decomposition occurs in accordance with reaction 5.3, where lithium evaporates. At the highest temperature,

$$
\begin{aligned}
& \mathrm{Li}_{2} \mathrm{O}(\mathrm{s})=\mathrm{Li}_{2} \mathrm{O}(\mathrm{g}), \\
& \mathrm{Li}_{2} \mathrm{O}(\mathrm{s})=2 \mathrm{Li}(\mathrm{g})+\frac{1}{2} \mathrm{O}_{2}(\mathrm{~g}),
\end{aligned}
$$

and probably

$$
\mathrm{Li}_{2} \mathrm{O}(\mathrm{s})=\mathrm{LiO}(\mathrm{g})+\mathrm{Li}(\mathrm{g})
$$

take place. These observations require that dilithium oxide also be formed during the decomposition process by

$$
\mathrm{Li}_{2} \mathrm{SO}_{4}(\mathrm{~s})=\mathrm{Li}_{2} \mathrm{O}(\mathrm{s})+\mathrm{SO}_{2}(\mathrm{~g})+\frac{1}{2} \mathrm{O}_{2}(\mathrm{~g}) \text {. }
$$

A new sample of lithium sulfate from an aqueous solution was evaporated onto the samplefilament. The temperature was slowly raised to $1050-1100^{\circ} \mathrm{K}$ over a period of three days. Continuous pumping removed the sulfur dioxide until its ion peak intensity was less than that of the residual nitrogen gas. The total pressure decreased to $9 \times 10^{-9}$ Torr. When the sample filament was heated to $1301^{\circ} \mathrm{K}$, lithium, $\mathrm{Li}_{2} \mathrm{O}$, and oxygen were found by electron-impact ionization. The $\mathrm{Li}_{2} \mathrm{O}$ ion mass 30 to mass 29 ratio was 5.8 .

On the basis of the thermodynamic data for $\mathrm{Li}_{2} \mathrm{O}$ available in the JANAF Tables, ${ }^{100}$ the calculated fluxes in molecules $\mathrm{cm}^{-2} \mathrm{sec}^{-1}$ for the species impinging on the hot filament are $Z_{\mathrm{Li}_{2} \mathrm{O}}=3.44 \times 10^{11}, \mathrm{Z}_{\mathrm{Li}}=$ $9.44 \times 10^{11}$, and $\mathrm{Z}_{\mathrm{Q}_{2}}=2.36 \times 10^{11}$. The vaporization coefficient was assumed to be unity in all cases. Lithium oxide was disregarded. The flux of oxygen from the residual gas is estimated to be $3.6 \times 10^{11}$ molecules $\mathrm{cm}^{-2} \mathrm{sec}^{-1}$, with a lesser contribution from the presence of sulfur dioxide.

Figure 36 shows the temperature dependence of the combined surface ionization of lithium and $\mathrm{Li}_{2} \mathrm{O}$. The observed ion currents were measured within 10 sec after flashing.

Above $1300^{\circ} \mathrm{K}$, complete dissociation of $\mathrm{Li}_{2} \mathrm{O}$ is apparent. The total ion current is the sum of the currents for lithium and $\mathrm{Li}_{2} \mathrm{O}$. As the temperature decreases, there is a sharp drop-off in current due to reevaporation of the $\mathrm{Li}_{2} \mathrm{O}$. On further decrease in temperature, the current drops, due to lithium adsorption on the surface.

This explanation for the observed ion current is not as clear-cut as suggested. It rests principally on the greater disparity between the expected 


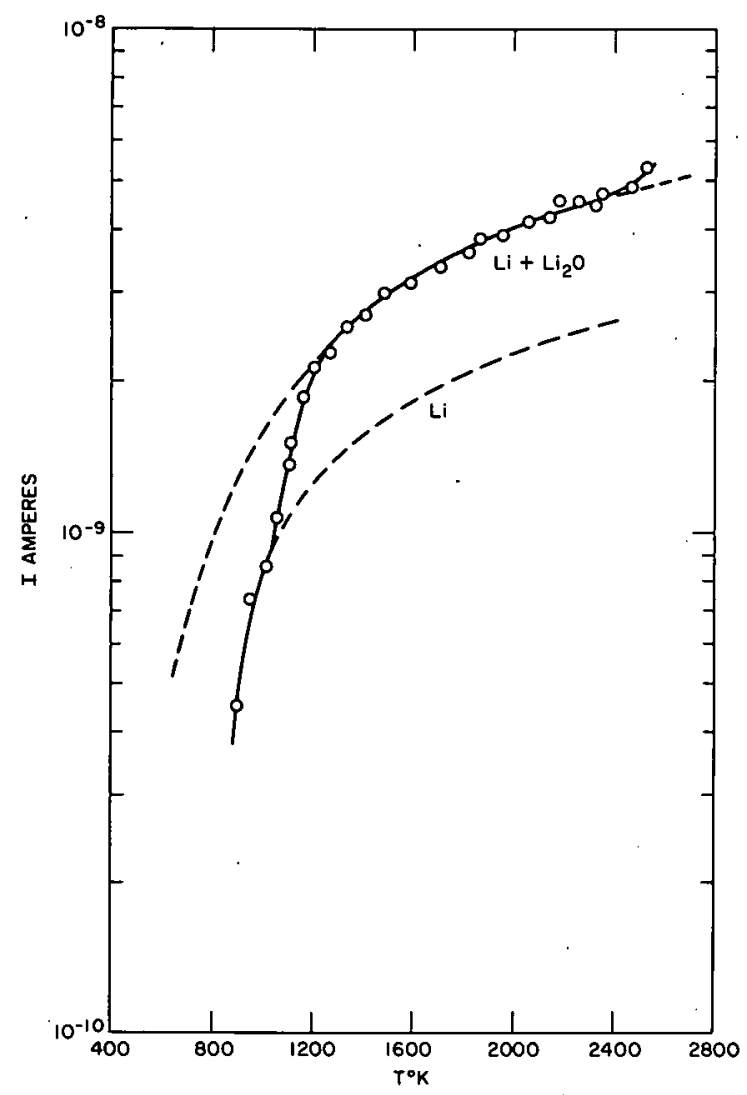

121-5507

Fig. 36 . Temperature Dependence of the Lithium Surface-ionization Current for Lithium and Dilithium Oxide on Rhenium curve (upper dashed line of Fig..36) and the experimental curve as compared to the results for surface ionization of lithium atoms only. For lithium atoms (Fig. 25), the ion current departs from the Saha-Langmuir curve at about $1150^{\circ} \mathrm{K}$. Next, the decrease in ion current with decreasing temperature is greater than that observed for the lithium halides. This is expected, the dissociation energy for the reaction

$\mathrm{Li}_{2} \mathrm{O}(\mathrm{g})=2 \mathrm{Li}(\mathrm{g})+\mathrm{O}(\mathrm{g})$

being greater. From the JANAF Tables, ${ }^{100}$ the calculated dissociation energy is $7.71 \pm 0.11 \mathrm{eV}$.

In addition, the re appears to be a decided cusp in the temperature dependence of the ion current at $1040^{\circ} \mathrm{K}$. This is ascribed to the onset of the surface ionization of lithium from dilithium oxide.

\section{E. The System Lithium Nitrate on Rhenium}

Thermodynamic data for the calculation of the vapor pressure of lithium nitrate are nonexistent. The ion intensities observed for a sample of lithium nitrate at a sample filament temperature of $640^{\circ} \mathrm{K}$ are $\mathrm{LiNO}_{3}^{+}(1)$, $\mathrm{Li}_{2} \mathrm{NO}_{3}^{+}(0.2), \mathrm{LiNO}_{2}^{+}(0.05), \mathrm{Li}^{+}(4), \mathrm{NO}_{2}^{+}(20), \mathrm{NO}^{+}(28)$, and $\mathrm{O}_{2}^{+}(6)$. This is a typical pseudohalide mass-spectral pattern obtained by electron-gun operation. Ion mass 30 (NO) did not contain any appreciable amount of $\mathrm{Li}_{2} \mathrm{O}$, since the ratio of mass 30 to mass 29 was greater than 100 .

\section{With a residual-gas background of $7.8 \times 10^{-9}$ Torr, the sample} filament was heated to $712^{\circ} \mathrm{K}$. This produced sufficient surface-ionization current to be readily measurable. The lithium surface-ionization current versus temperature is shown in Fig. 37. The plotted points correspond to the current obtained within $10 \mathrm{sec}$ after flashing the surface-ionization filament to $2432^{\circ} \mathrm{K}$.

The small difference in temperature for the onset of lithium adsorption for lithium nitrate $\left(1180^{\circ} \mathrm{K}\right)$ and for lithium atoms $\left(1150^{\circ} \mathrm{K}\right)$ is not 


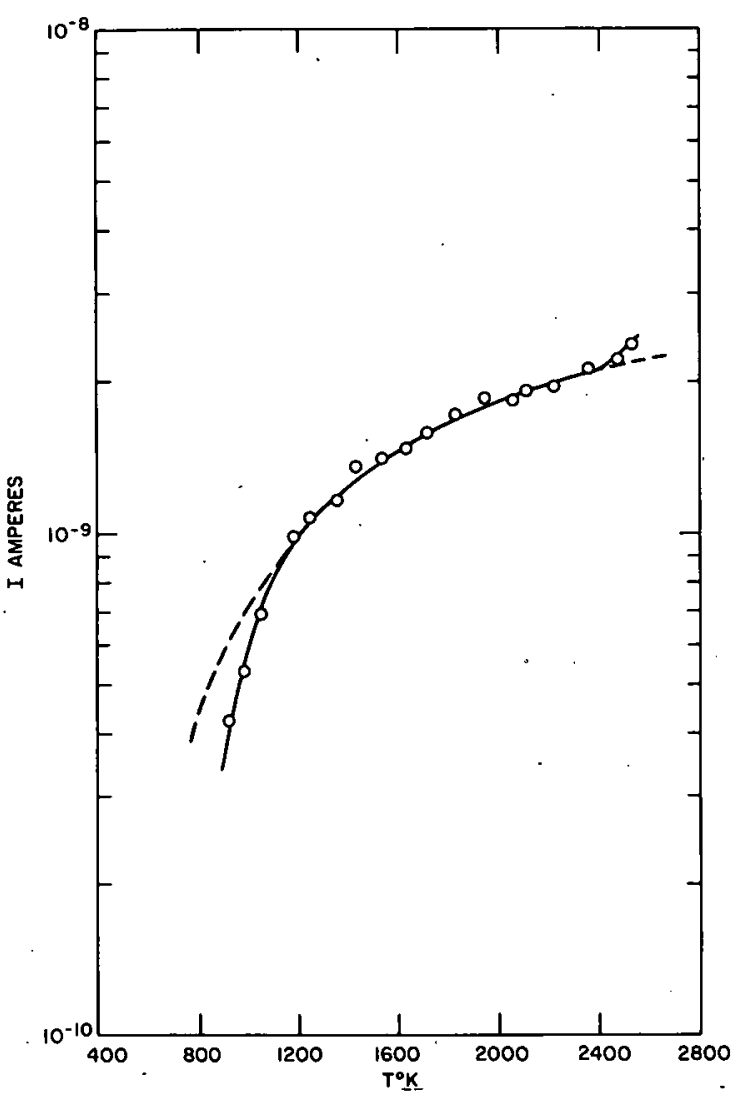

$121-5506$

Fig. 37. Temperature Dependence of the Lithium Surface-ionization Current for the System Lithium Nitrate on Rhenium considered significant. The data suggest complete dissociation of lithium nitrate over the entire temperature range.

When the surface-ionization current was allowed to approach equilibrium at temperatures below $1600^{\circ} \mathrm{K}$, the rate of ion-current equilibration was more rapid than expected for the low residual-gas pressure in the system. At $1323^{\circ} \mathrm{K}$, equilibrium ionization was attained in $36 \mathrm{~min}$. Flashing the filament to $2500^{\circ} \mathrm{K}$ and using electron-impact techniques revealed a series of lithium rhenates. The ion masses corresponded to $\mathrm{Li}_{2} \mathrm{ReO}_{4}$, LiReO ${ }_{4}$, and $\mathrm{LiReO}_{3}$, together with $\mathrm{ReO}_{3}$ and $\mathrm{ReO}_{2}$.

The interaction between lithium nitrate and rhenium can account for the temperature dependence of the ion current. However, the possibility exists that lithium nitrate is thermodynamically unstable with respect to dissociation for all surface-ionization filament temperature.

The more rapid rate of approach to ion-current equilibration for lithium nitrate as opposed to lithium atoms is construed to be a result of the reaction of lithium nitrate with rhenium. 
CHAPTER VI

\section{SUMMARY AND CONCLUSIONS}

A combination of high-vacuum, flash-desorption, and massspectrometric techniques was applied to the study of the surface ionization of lithium and selected lithium salts on polycrystalline rhenium filaments. To ensture an atomically clean surface, high vacuum (pressure $<10^{-8}$ Torr) and flash desorption of adsorbed gases prior to current measurements were used. The species present, both in the flux of particles impinging on the surface-ionization filament and in the desorbed gas, were analyzed massspectrometrically.

A timing circuit for flashing the filament to high temperatures was constructed to initiate the start and control the duration of heating. The flux of molecules impinging on the surface-ionization filament was determined from their thermodynamic properties and a measured temperature using a resistance thermometer. A Maxwell ac bridge was constructed for measuring the sample-filament resistance. When the bridge was coupled with an oscilloscope-rotating-trace method of null detection, a sensitive and accurate resistance thermometer was obtained for temperatures up to $1000^{\circ} \mathrm{K}$.

Rhenium was chosen for the surface-ionization studies due to its relatively low chemical activity compared to tungsten, tantalum, or molybdenum. Among the most interesting chemical properties of rhenium are its reactions with oxygen, nitrogen, water vapor, and carbon-containing compounds usually present in the composition of residual gases in-the massspectrometer source. A combination of flash desorption and mass spectrometry was used to measure the sticking coefficient of nitrogen on rhenium. At room temperature, the sticking coefficient was 0.008 . The measured value for the sticking coefficient with the rhenium filament at $752^{\circ} \mathrm{K}$ was 0.001 . These values agree with those obtained by Scheer and McKinley ${ }^{118}$ $\left(0.008\right.$ at $\left.300^{\circ} \mathrm{K}\right)$ and by Gasser and Thwaites ${ }^{174}\left(0.0024\right.$ at $291^{\circ} \mathrm{K}$ and 0.0006 at $\left.870^{\circ} \mathrm{K}\right)$.

A perusal of the literature shows that the sticking coefficients of the other residual gases on rhenium are low, except for oxygen. Flash desorption of oxygen from metals has generally proved unsuccessful for obtaining precise surface-coverage and sticking-coefficient data. At high coverage, the desorbed species contain appreciable metal oxides. This was verified by electron impact ionization of the species desorbed on flashing the rhenium filament to $2500^{\circ} \mathrm{K}$. Ion masses corresponding to rhenium, $\mathrm{ReO}_{2}$, and $\mathrm{ReO}_{3}$ were observed.

The effective surface-ionization work function obtained for lithium on polycrystalline rhenium was $5.21 \pm 0.01 \mathrm{eV}$. The measured effective 
thermionic-emission work function was $4.98 \pm 0.03 \mathrm{eV}$. The difference between these work-function values gives a measure of the degree of contrast of the emitter surface. Patches with high work functions contribute more to the positive surface-ionization current than do low-work-function patches. The principal contribution to the electron current in thermionic emission arises from the low-work-function patches.

The validity of the Saha-Langmuir equation in the range of $1200-2600^{\circ} \mathrm{K}$ was demonstrated.for the lithium-rhenium system. Such an agreement over the wide temperature range used has not been obtained for other systems or by other investigators. In the older studies under high-vacuum conditions, the equilibrium-ionization current was usually measured, resulting in ion currents from a contaminated surface.

Below $1.200^{\circ} \mathrm{K}$, the lithium surface-ionization current was less than expected as based on the Saha-Langmuir equation. This was attributed to lithium adsorption on rhenium, lowering the work function.

Flashing the surface-ionization filament to $2400^{\circ} \mathrm{K}$ had no effect on the lithium surface-ionization current for filament temperatures above $1500^{\circ} \mathrm{K}$. Below $1500^{\circ} \mathrm{K}$, the ion current increased with time after flashing, attaining a steady-state value. The rate of attainment of the steady-state current was demonstrated to be due to oxygen adsorption, increasing the work function.

An analysis of the time required to àpproach a given fraction of the steady-state ion current as a function of the surface-ionization filament temperature in the range of $1150-1450^{\circ} \mathrm{K}$ yielded the desorption energy of oxygen atoms on rhenium. The desorption energy obtained for the most energetic adsorption center on rhenium was $4.13 \mathrm{eV}$. A number of simplifying assumptions were used, such as adsorption centers of different strengths and ignoring the variation of the desorption energy and dipole moment with coverage. Although the value for the desorption energy of oxygen atoms on rhenium is subject to large errors, the method.illustrates the use of surface-ionization techniques for obtaining the desorption energy of oxygen on refractory metals.

At surface-ionization filament temperatures below $1100^{\circ} \mathrm{K}$, the rate of attainment of ion-current equilibration was not a smooth function of time, showing considerable structure. This was attributed to the superposition of adsorption curves corresponding to more than one type of adsorption center. It may also reflect competition between lithium and oxygen for the adsorption sites.

The effect of the anionic constituents on the surface ionization of lithium chloride, lithium bromide, lithium iodide, lithium sulfate, and lithium 
nitrate was investigated. Above a certain temperature, the temperature dependence of the lithium surface-ionization current for atomic lithium, lithium bromide, and lithium chloride was the same; this temperature is higher for chloride than for bromide. The threshold ionization temperatures for atomic beams was lower than for the molecular beams; the difference of these temperatures increases from bromide to chloride. These results can be explained on the hypothesis of complete dissociation of the molecules of lithium bromide and lithium chloride on rhenium in the high-temperature region and reduced dissociation of the molecules in the region of threshold temperatures.

The temperature dependence of the ion current for two different intensities of the incident molecular beam can be used to obtain the dissociation energy of the gaseous molecule. The dissociation energy for lithium chloride was $\mathrm{D}_{\mathrm{LiCl}(\mathrm{g})}=4.9 \pm 0.1 \mathrm{eV}$ in the range of $1300-1700^{\circ} \mathrm{K}$. For lithium bromide, $\mathrm{D}_{\mathrm{LiBr}}(\mathrm{g})=4.4 \pm 0.1 \mathrm{eV}$ in the range of $1150-1400^{\circ} \mathrm{K}$. The values obtained from the JANAF Tables ${ }^{100}$ at $1300-1700$ and $1150-1400^{\circ} \mathrm{K}$ are $5.02 \pm 0.13 \mathrm{eV}$ and $4.47 \pm 0.13 \mathrm{eV}$, respectively.

The temperature dependence of the surface-ionization current from lithium iodide beams differing by a factor of 30 in intensity was identical to the temperature dependence of the current obtained for atomic beams of lithium. On the basis of thermodynamic data, the lithium surface-ionization current was expected to decrease at about $1320^{\circ} \mathrm{K}$ for the lithium iodide beam of highest intensity, due to incomplete dissociation. This was not observed. It was postulated that iodide reacted with rhenium, shifting the dissociation equilibrium to lower temperatures. This could not be verified by electron-impact study of the desorbed species on flashing. No ion masses corresponding to rhenium iodides or oxyiodides could be found. This does not preclude, however, a shift in the dissociation equilibrium by rhenium abstraction and subsequent emission of iodine.

Lithium sulfate decomposed in part to dilithium oxide. Beams of lithium, oxygen, and dilithium oxide of comparable densities arose from $\mathrm{Li}_{2} \mathrm{O}$ on heating. Above $1300^{\circ} \mathrm{K}$, the surface-ionization current was a composite current from the atomic lithium beam and the completely dissociated $\mathrm{Li}_{2} \mathrm{O}$ beam. The decrease in ion current with decreasing temperature beginning at $1250^{\circ} \mathrm{K}$ is due to incomplete dissociation of $\mathrm{Li}_{2} \mathrm{O}$. At $1150^{\circ} \mathrm{K}$, the ion current decreases due to lithium adsorption as found for pure lithium atomic beams.

The surface ionization of lithium nitrate shows complete dissociation of the nitrate over the temperature range used. The rate of ion-current equilibration below $1600^{\circ} \mathrm{K}$ after flashing was more rapid than expected for the low residual-gas pressure present. A definite surface reaction between lithium nitrate and rhenium took place. Electron-impact ionization of the species desorbed on flashing revealed a series of lithium rhenates with ion masses corresponding to $\mathrm{Li}_{2} \mathrm{ReO}_{4}, \mathrm{LiReO}_{4}$, and $\mathrm{LiReO}_{3}$. 
Anionic constituents of lithium salts can influence the lithium surface-ionization current obtained in several ways. Incomplete dissociation of lithium chloride and lithium bromide resulted in reduced lithium ion currents at surface-ionization filament temperatures well above the threshold temperature for the surface ionization of lithium atoms. A chemical reaction between rhenium and iodine in the surface ionization of lithium iodide is suggested for the enhanced lithium ion emission observed in the temperature range where incomplete dissociation of lithium iodide was expected. For lithium nitrate incident on rhenium, lithium rhenates were formed. The lithium ion current was similar to that obtained for atomic lithium beams. Lithium sulfate decomposed on heating to give beams of dilithium oxide and atomic lithium. The temperature dependence of the total lithium surfaceionization current was the sum of the ion currents for the individual beams. 


\section{ACKNOW LEDGMENTS}

I express my gratitude to Professor Phillip G. Wahlbeck of the Illinois Institute of Technology and to Dr. Martin H. Studier of the Argonne National Laboratory for their guidance, encouragement, and friendship.

I. acknowledge my. indebtedness and sincere appreciation to the - Argonne National Laboratory for. my support and for the privileges. and excellent services accorded me. In this regard, I thank the management and personnel of the Chemistry Division Shops. Special thanks is given to Mr. William K. Brookshier and Mr. Albert A. Longnecker of the Electronics Division for help and ideas in the design of the various electrical circuitry.

I appreciate the advice and help of all my associates in the Laboratory and, in particular, the aid given by Mr. Leon Moore, Mrs. Alberta Martin, Mrs. Emily White, and the members of Dr. Robert J. Thorn's group. 


\section{REFERENCES}

1. H. S。W. Massey and E. H. S. Burhop, Electronic and Ionic Impact Phenomena, Oxford, Clarendon Press (1952), Chapter 9.

2. Manfred Kaminsky, Atomic and Ionic Impact Phenomena on Metal Surfaces, Academic Press, Inc., New York (1965)。

3. J. B. Hasted, Physics of Atomic Collisions, Butterworths, London (1964).

4. G. Ehrlich, Ann. Rev. Phys。Chem. 17, 295 (1966).

5. Mo G。 Inghram and R. J. Hayden, A Handbook on Mass Spectrometry, Nat1. Acad. Sci.--Nat1。Res。Council, Nucl. Sci. Ser., Rept. 14 (1954).

6. M。H。Studier, E。 No Sloth, and L. P. Moore, J. Phys. Chem. 66, 133 (1962)。

7. J。 R。 Werning, Ph.D. thesis, University of California (1958) (UCRL-8455).

8. E. Ya. Zandberg and A. Ya。Tontegode, Soviet Phys.--Tech. Phys. (English transl。) 10, 858 (1965)。

9. T。W。 Richardșon, Phil。Mag。6, $86^{\prime}$ (1903).

10. R. H. Plumlee and L. P. Smith, J. App1。 Phys. 21, 811 (1950).

11. S. Datz and E. H. Taylor, J. Chem。 Phys. 25, 395 (1956).

12. F. L. Reynolds, J。Chem. Phys。39, 1107 (1963)。

13. M. J. Dresser and D. E. Hudson, Phys. Rev. 137, A673 (1965).

14. L. N. Dobretzov, Elektronen ind Ionenemission, VEB-Verlag Technik, Berlin (1954)。

15. M. J. Copley and T.E。Phipps, Phys。Rev。45, 344. (1934); 48, 960 (1935)。

16. G. Ehrlich, Ann. N. Y. Acad。Sci。101, 722 (1963).

17. J. B. Taylor and I. Langmuir, Phys, Rev。44, 423 (1933).

18. I. Langmuir and $\mathrm{K}$. H。Kingdon, Proc. Roy. Soc. (London), A10?, 61 (1925)。

19. I. Langmuir, J。Am. Chem.Soc。40, 1361 (1918)。

20. E. Ya. Zandberg and N. I. Ionov, Soviet Phys。--Usp。 (English transl.) 2, 255 (1959).

21. A. N. Romanov, Soviet Phys。-Tech。Phys. (English trans1.) 2, 1125 (1957).

22. E。Ya. Zandberg and N。I. Ionov, Soviet Phys.-Tech. Phys. (English transl.) 3, 2243 (1958).

23. S。 Datz and E. H. Taylor, JoChem。 Phys. 25, 389 (1956).

24. Kim Heng Pong and I. L. Sokolskaya, Bull. Leningrad State Review, No. $12,65^{\circ}(1952)$.

25. Mo Kaminsky, Ann. Physik。18, 53 (1966)。

26. 0. Knacke and I。NoStranski, Progr. Metal Phys. 6, 181 (1956). 
27. G. F. Smith, Phys。Rev。94, 295 (1954).

28. A。 Ro Hudson, Phys。Rev。98, 889 (1955).

29. J。A。Becker and C. D. Hartman, J. Phys。Chem. 57, 153 (1953).

30. H. D. Hagstrum, Rev。Sci。Instr。24, 1122 (1953).

31. H. D. Hagstrum, Phys. Rev. 98, 561 (1955)。

32. A. Eberhagen, Fortschr。 Phys. 8, 245 (1960).

33. G。 Ehrlich and F。 G。 Hudda, J。Chem. Phys。35, 1421 (1961).

34. T. Oguni, J. Phys。 Soc。, Japan, 19, 83 (1964).

35. A. A. Holscher, J。 Chem. Phys。41, 579 (1964).

36. A。G。 J。 van Oostrom, Philips Res。 Rep。Suppl。 No. 1 (1966).

37. F。 H。 Hayes, M。 P。Hi11, S. M. A. Lecchini, and B. A. Pethica,

J. Chem。 Phys. 42, 2919 (1965)。

38. T。A。 Delehar and Go Ehrlich, J。Chem. Phys。42, 2686 (1965).

39. J. Frenke1, J. Exp. Theoret. Phys。(USSR) 2, 706 (1941).

40. D. O. Hayward and B. M. W. Trapnell, Chemisorption, Butterworths, Washington, D。C。, 2nd.Edition, 1964, p. 147.

41. J。Becker, Ann. N.Y. Acad。Sci。58, 721 (1954).

42. N。S。 Rasor and CoWarner, J. App1。Phys. 35, 2589 (1964).

43. R。W. Gurney, Phys。Rev, 47, 479 (1935).

44。 S。V。 Starodubtsev, J. Exp. Theoret. Phys. (USSR) 19, 215 (1949).

45. J. D. Levine and E。 P。Gyftopoulos, Surface Sci. 1, 225 (1964).

46. M。 Kaminsky, Advan. Mass Spectrometry, Proc. Conf. 3rd, Paris, 1964 (1966), p. 295.

47. M。 D。 Scheer and J. Fine, J。 Chem. Phys。37, 107 (1962).

48. M。 D。 Scheer and J. Fine, J。Chem. Phys: 38, 307 (1963)。

49. F。 L。 Hughes, Phys。Rev。113, 1036 (1959).

50. F。 L。 Hughes and H. Levinstein, Phys. Rev. 113, 1029 (1959).

51. E. W. Mưller, Ergeb。Exakt. Naturw. 27, 290 (1953).

52。 G。Ehrlich, J。Chem. Phys。31, 1111 (1959)。

53. G. Ehrlich, Structure and Properties of Thin Films, Ed. by C. A. Neugebauer, J. B. Newkirk, and D。A。 Vermilyea, John Wiley and Sons, Inc., New York (1959), pp。423-471.

54. L. Apker, Ind. Eng. Chem。40, 846 (1948).

55. R。Gomer, "Chemisorption,".Fundamentals of Gas-Surface Interactions, Ed. by H.Saltsburg, J。 N。 Smith, and M. Rogers, Academic Press, Inc., New York (1967), p. 182.

56. T。E。 Madey and J。 T。 Yates, J。Chem. Phys。44, 1675 (1965).

57. G. Ehrlich, J. Appl。 Phys. 32, 4 (1961). 
58. P。A. Redhead, Vacuum 12, 203 (1962).

59. G。 Ehrlich, J。Chem. Phys。36, 1171 (1962)。

60. A. A. Bell and R。Gomer, J。Chem. Phys. 44, 1065 (1966).

61. T. E. Madey, J. T. Yates, and R。 C. Stern, J. Chem. Phys. 42, 1372 (1965)。

62. R. Gomer, Discuss. Faraday Soc. 41, 14 (1966)。

63. R. Gomer and L. W. Swanson, J. Chem。 Phys. 38, 1613 (1963).

64. L。 D。 Schmidt and R。Gomer, J。Chem. Phys。42, 3573 (1965).

65. L. D. Schmidt and R. Gomer, J. Chem。. Phys, 45, 1605 (1966).

66. R。 P。Redhead, Trans。Faraday Soc. 57, 641 (1961).

67. L. J. Rigby, Can. J. Phys。42, 1256 (1964)。

68. J. Eisinger, J。 Chem. Phys。27, 1206 (1957)。

69. V. M. Gavrilyuk and V. K. Medvedev, Soviet Phys.-Solid State (English trans1.) 4, 1737 (1963)。

70. Yu。 $\mathrm{K}$. Ustinov, $\mathrm{V} 。 \mathrm{~N}$. Ageev, and N. I. Ionov, Soviet Phys.-Tech. Phys. (English transI。) 10, 851 (1965)。

71. L. W. Swanson and R. Gomer, J. Chem。 Phys. 39, 2813 (1963).

72. D。 Menzel and R. Gomer, J. Chem。 Phys. 41, 3329 (1964).

73. J。W. May and L。H.Germer, J. Chem。 Phys. 44, 2895 (1966).

74. I。 Langmuir and K. H. Kingdon, Science 57, 58 (1923).

75. W. Weiershausen, Ann. Physik. 15, 30 (1965).

76. D。B.Langmuir, Phys。Rev。49, 428 (1936).

77. J。G。Potter, Phys。Rev. 58, 523 (1940).

78. A。 L。 Reimann, Proc. Roy. Soc. (London) 163, 499 (1937).

79. W。 B。 Nottingham. Phys。Rev。41, 793 (1932).

80。 C. Herring and M. H。 Nichols, Rev。Mod。Phys. 21, 185 (1949).

81. R. H. Fowler, Phil. Mag. 45, 1 (1923).

82。 G。 A。 Morosow, Zh. Tekhn. Fiz. 17, 1143 (1947).

83. Wo Schottky, Physik Z. 15, 872 (1914)。

84. A. N. Romanov and S. V. Starodubtsev, Soviet Phys.-Tech. Phys. (English trans1.) 2, 652 (1957)。

85: J. Zemel, J. Chem。 Phys. 28, 410 (1958)。.

86. I. N. Bakulina and No. I。 Ionov, Soviet Phys. JETP (English transl.)

9, 709 (1959).

87. K。 Ho Kingdon, Phys。Rev。24, 510 (1924).

88. I。 Langmuir and K. H. Kingdon, Phys。Rev。34, 129 (1929).

89. W。Walcher, Z。 Physik. 121, 604 (1943). 
90。 J。 Eisinger, J. Chem。Phys. 28, 165 (1958); 29, 1154 (1958); 30, 412 (1959).

91. A. van Oostrom, J. Appl。Phys. 33, 2917 (1962).

92.W。Weiershausen, Ann. Physik 15, 252 (1965)。

93. I. Langmuir, J。Am. Chem。Soc。54, 2798 (1932)。

94. M. N. Saha, Phil. Mag. 46, 534 (1923)。

95. L. J. Varnerin, Phys. Rev. 91, 859 (1953)。

96. H. D. Hagstrum, Phys. Rev. 96, 336 (1954)。

97. I. Frenke1, Z. Physik 26, 117 (1924)。

98. N. D. Potekhina, Soviet Phys。-Tech。Phys. (English trans1.) 10, 1280 (1966).

99. T。 L。 Bailey, J. Chem。 Phys. 28, 792 (1958)。

100. JANAF Thermochemical Data, Ed。by DoR. Stu11, The Dow Chemical Company, Midland, Michigan (1962)。

101. S。V。Starodubtsev, Tr。Fiz。-Tekhn. Inst。Akad. Nauk。Vzber。SSR 2,6 (1948).

102. G. M. Pyatigorskii, Soviet Phys.-Tech. Phys。(English transl.) 10, 867 (1965).

103. M. D. Scheer and J.Fine, J. Chem。 Phys. 36, 1647 (1962).

104. K. K. Kelley, U。S。Bur。Mines Bull。 584 (1960)。

105. K。K。 Kelley and G. G. King, U.S。Bur。 Mines Bull。 592 (1961).

106. C。E.Wicks and F。E。Block, U。S。Bur。Mines Bul1。605 (1963).

107. D。 R。 Stull and G。 Sinke, Advan. Chem. Ser. 18 (1956).

108. An. N. Nesmeyanov (Transl. and Edit. by J. I. Carasso), Vapor Pressure of the Elements, Academic Press, Inc。, New York (1961).

109. R。 Hultgren, R. Orr, P. Anderson, and K. K. Kelley, Selected Values of Thermodynamic Properties of Metals and AlZoys, John Wiley and Sons, Inc。, New York (1963).

110. J. W. Green, D. E. Poland, and J. L. Margrave, Jo Chem. Phys. 33, 35 (1960); 36, 1088 (1962)。

111. D。 D. Wagman, W. H. Evans, S. Levine, and I. Jaffey, Nat. Bur. Std. (U.S.) Circ. 500 (1952).

112. Annual Review of Physical Chemistry (Ed。H。Eyring), Annual Reviews Inc., Palo Alto, California, Vol. 17, 1966.

113. M. H. Nichols, Phys. Rev, 57, 297 (1940).

114. E. W. Muiller, Phys. Rev. 96, 837 (1954); J。 Appl. Phys. 26, 732 (1955).

115. A. van Oostrom, Phys. Letters 4, 34 (1963):

116. L. K. Hanson, J。 Appl. Phys。37, 4498 (1966)。

117. G。 Popp, Ann. Physik。 13, 115 (1964). 
118. M. D. Scheer and J. D. McKinley; Surface Sci. 5, 332 (1966).

119. G. Ehrlich, Advan。Catalysis 14, 256 (1963).

120. E. P. Gyftopoulos and J. D. Levine, J. App1. Phys. 33, 67 (1962); Surface Sci. 1, 171, 225, 349 (1964).

121. J. W. Gadzuk, Surface Scci. 6, 133, 159 (1967).

122. J.H. DeBoer, Advan, Catalysis. 8, 17 (1956).

123. J. W. Gadzuk and E。N。Carabateas, J. Appl. Phys. 36, 357 (1965).

124. Jo Ro. MacDonald and C. D。 Barlow, Jro, J. Chem. Phys. 40, 1535 (1964); 43, 2575 (1965); 44, 202 (1966)。

125. J。W. Gadzuk, Proc. 25th Ann. Conf. Phys。 Electronics, M.I.T. (March 1965)。

126. I. Tamm, Physik Z. Sowjetunion 1, 733 (1932).

127. Wo Shockley, Phys。:Rev。56, 317 (1939).

128. J. D. Levine and P。 Mark, Phys, Rev. 144, 751 (1966).

129.J。Koutecky; Surface Scị。1, 280 (1964).

130。 J. Koutecky, Advan. Chem。 Phys. 9, 85 (1965)。

131. J。Koutecky, Phys。Chem, Solids 14, 233 (1960).

132. J。Koutecky, Angew, Chem. Intern, Ed. Eng1. 3, 496. (1964).

133. T. B. Grimley, Advan. Catalysis 12, 1 (1960).

134. T. B. Grimley, Phys. Chem. Solids 14, 227 (1960).

135. M。 F。 Deigen and M. D. Glinchuk, Surface Sci. 3, 243 (1965).

136. V. Heine, Phys. Rev. 138, A1689 (1965).

137. P. Mark, Catalysis Rev. 1, 165 (1967).

138. W. C. Wiley, Science 124,217 (1956).

139. D. B. Harrington, Encyclopedia of Spectroscopy, Reinhold Publ. Corp。, New York (1960), pp。628-647。

140。 D。C. Damoth, Advan: Anal. Chem. Instr. 4, 371 (1964).

141. M. H. Studier, Rev. Sci. Instr。34, 1367 (1963).

142. J。 R。 Haumann and M。 Ho Studier, Rev。Sci. Instr. 39, 169 (1968).

143. H. A. Jones and I. Langmuir, Gen. Elec. Rev. 30, 310 (1927).

144. W. E. Forsythe and A. C. Worthing, Astrophyș. J. 61, 126 (1925).

145. M. P. Hill, S。 M。 A。 Lecchini, and B。A。 Pethica, Trans. Faraday Soc, 62, $229(1966)$.

146. H。F。 Stimson, J. Res. Nat. Bur. Std. 65A, 139 (1961):

147. C. T. Sims, C. M。 Craighead, and R. I. Jaffee, J。 Metals 7, 168 (1955)。

148. F。 R。 Caldwe11, Nat. Bur. Std. (U.S。) Monograph 40 (1962). 
149. R. Suhrmann, Advan。 Catalysị.7, 303, 316 (1955).

150. H. Mayer, Naturwissenschaften 26, 28 (1938)。

151. To Mo Dauphinee, "Potentiometric Methods of Resistance Measurements," Temperature, Its Measurement and Control in Science and Industry, Ed。by F。G。Brickwedde, Reinhold Publ. Corp., New York, 1962, Vol. 3, Part 1, p. 259.

152. H. L. Daneman and G. C. Mer̀gner, Instruments Technology 14, 51 (May 1967); 14, 65 (June 1967)。

153. J。Czech, OscizZoscope Measuring Technique, Springer-Verlag, New York (1965), Chapter 8.

154. H. V. Malmstadt and C. G. Enke, Electronics for Scientists, W. A。 Benjamin, Inc., New York (1962), p。281; B。 I. Bleaney and B. Bleaney, Electricity and Magnetism, Oxford, Clarendon Press (1957), p. 464 .

155. H. J. Kostkowski and R。 D。 Lee, Theory and Methods of Optical Pyrometry, Ref. 151, p. 449.

156. D. R. Lovejoy, Recent Advances in Optical Pyrometry, Ref. 151, p. 487 。

157. R。 J。 Thorn and G。H.Winslow, Am. Soc. Mech. Eng: Paper 63-WA-224 (1963).

158. 0. D. Judd, Nat。 Bur。Std。 Res。 Papers No. 289 (1931).

159. D. T. F. Marple, J. Opt。Soc。Am. 46, 490 (1956).

160。 R。 T。 Birge, Am. J. Phys。?, 351 (1939)。

161。 E.Ya. Zandberg and A。Ya. Tontegode, Soviet Phys.-Tech. Phys. (English transl。) 11, 713 (1966)。

162. G。 B。 Gaines, C. T. Sims, and R. I. Jaffee, J。 Electrochem. Soc. 106, 881 (1959).

163. H. Hahn and A. Konrad, Z. Anorg. Allgem。Chem. 264, 174 (1951).

164. G. Barnes, Phys. Rev. 97, 1579 (1955).

165. W. Weiershausen, Ann。 Physik. 15, 150 (1965)。

166. Mo Ho Studier, J。 Phys. Chem。66, 189 (1962).

167. F. Skoupy, Z。 Tech. Physik。5, 563 (1924)。

168: Wo Jost, Diffusion, Academic Press, Inc., New York (1960), p. 324.

169. E。 R。Plante and R. Szwarc, J。Res。Nat。Bur. Std. A, 70, 175 (1966).

170. J. B. Mann, J。 Chem. Phys。46, 1646 (1967)。

171. A。 Go Harrison, E. G. Jones, S. K. Gupta, and G. P。Nagy, Can. J. Chem。44, 1967 (1966)。

172. W。 Ploch and W. Walcher, Rev。Sci。Instr. 22, 1028 (1951).

173. G。E. Moore and F。C. Unterwald, J。Chem. Phys. 40, 2639 (1964). 
174. R. P. H. Gasser and R. Thwaites, Vacuum 17, 265. (1967).

175。 $R_{0} P_{0} H_{0}$ Gasser, K. Roberts, and $R_{0}$ Thwaites, Trans. Faraday Soc. 63,2765 (1967)。

176。 R。 P。H. Gasser, R. Thwaites, and J. Wilkinson, Trans. Faraday Soc. . 63, 195 (1967).

177。 K。 F。 Poulter and J。A。Pryde, Brit. J. Appl。Phys. (J. Phys. D.) 1, 169 (1968)。

178. H。Utsugi and R. Gomer, J. Chem。 Phys. 37, 1706 (1962).

179. L. H. Taylor, Surface Scị. 2, 188 (1964).

180. C. M. Quinn and M. W. Roberts, Proc。Chem. Soc. 246 (1962).

181. J. Anderson, W。 E。 Danforth, and A。 I。 Williams, J. App1. Phys. 34, 2260 (1963).

182. R. G. Wilson, J。App1. Phys。37, 3170 (1966)。

183. N。 Io. Alekseev and D. L. Kaminskii, Soviet Phys.-Tech. Phys. (English trans $1_{0}$ ) 9, 1177 (1964)。

184. E。Ya. Zandberg and A。Ya. Tontegode, Soviet Phys。-Tech. Phys. (English tranșlo) 10, 260 (1965).

185. W. Weiershausen, Advan. Mass Spectrometry, Proc。Conf. 1st, London, 1959 (1961), p. 120.

186. A. Buchler and J. B. Berkowitz-Mattuch, Advan. High Temp. Chem. 1, 95 (1967)。

187. P。 Jo Ficalora, O. M. Uy, D。W. Muenow, and J。 L. Margrave, J. Am : Cer。Soc。51, 574 (1968),

188. V. I. Spitsyn and. V. I. Shostak, J. Gen. Chem。USSR (English Trans1.) 19, 251 (1949).

189. J. Berkowitz, W. A. Chupka, C。D。Blue, and J。L。 Margrave, J. Phys. Chem。63, 644 (1959). 